\title{
LARGE POPULATION LIMIT AND TIME BEHAVIOUR OF A STOCHASTIC PARTICLE MODEL DESCRIBING AN AGE-STRUCTURED POPULATION
}

\author{
VIET CHI TRAN ${ }^{1}$
}

\begin{abstract}
We study a continuous-time discrete population structured by a vector of ages. Individuals reproduce asexually, age and die. The death rate takes interactions into account. Adapting the approach of Fournier and Méléard, we show that in a large population limit, the microscopic process converges to the measure-valued solution of an equation that generalizes the McKendrick-Von Foerster and Gurtin-McCamy PDEs in demography. The large deviations associated with this convergence are studied. The upper-bound is established via exponential tightness, the difficulty being that the marginals of our measure-valued processes are not of bounded masses. The local minoration is proved by linking the trajectories of the action functional's domain to the solutions of perturbations of the PDE obtained in the large population limit. The use of Girsanov theorem then leads us to regularize these perturbations. As an application, we study the logistic age-structured population. In the supercritical case, the deterministic approximation admits a non trivial stationary stable solution, whereas the stochastic microscopic process gets extinct almost surely. We establish estimates of the time during which the microscopic process stays in the neighborhood of the large population equilibrium by generalizing the works of Freidlin and Ventzell to our measure-valued setting.
\end{abstract}

Mathematics Subject Classification. 60J80, 60K35, 92D25, 60F10.

Received 12 September, 2007.

\section{INTRODUCTION AND MOTIVATIONS}

Structured population models describe the dynamics of populations in which individuals differ according to variables that affect their reproductive capacities and survivals. In this article, we are interested in a population structured by ages. Age-structures are important to take into account the changes of behaviour of an individual during its life as well as life histories. It is natural to consider many ages. Examples are the physical age (the time since birth), the biological age (the intrinsic maturation stage of the individual), the age of an illness (the time since infection), the stage of the illness (the clinical stage of the illness). To our knowledge, the literature on the subject mostly considers structuration by the only physical age.

Our purpose is to study a microscopic stochastic population structured by a vector of ages that can grow nonlinearly in time and which models the age-dependence of the birth and death rates as well as possible

\footnotetext{
Keywords and phrases. Age-structured population, interacting measure-valued process, large population approximation, large deviations, exit time estimates, Gurtin-McCamy PDE, extinction time.

${ }^{1}$ Université Paris X-Nanterre, Équipe Modal'X, bâtiment G, 200 avenue de la République, 92100 Nanterre Cedex, France; vtran@u-paris10.fr
} 
interactions between individuals (competition or cooperation), including competition of logistic type (for which the death rate is linear in the size of the population).

Continuous time physical age-structured models that generalize the models of Malthus [33] and Verhulst's famous Logistic Equation [48], have made the object of an abundant literature based on the theory of partial differential equations (PDEs) (see Sharpe and Lotka [45], McKendrick [35], Gurtin and MacCamy [22], Marcati [34], Busenberg and Iannelli [6], Webb [51]).

Stochastic models generalizing the Galton-Watson process [19] have been studied by Bellman and Harris [3,23], Athreya and Ney [2] Chap. IV). These models consider non Markovian processes, called agestructured branching processes, structured by the physical age and in which the lifelength of an individual does not follow an exponential law. Each particle, at its death, is replaced by a random number of daughters with a law that does not depend on the age of the mother nor on the state of the population.

The assumptions of birth at the parent's death and of non dependence between the reproduction law and the age of the parent are biologically restrictive. Kendall [30], Crump and Mode [9,10], Jagers [26] and Doney [13] have studied birth and death processes in which a particle can give birth many times during its life, with a rate that depends on its age.

In the preceding models, the particles alive at the same time are independent, which is also a biologically restrictive assumption. Wang [50], Solomon [46] consider birth and death processes in which lifelengths are independent, but where the birth rate of a particle depends on the state of the population. Oelschläger [39], Jagers and Klebaner [27], generalize their works to take interactions in the birth and death rates into account. However, these rates remain bounded, which excludes interactions of logistic type.

We present here an individual-centered model which takes age-structure into account. We follow the works of Fournier and Méléard [17] and Champagnat et al. [7,8]. Our paper is drawn on more general models of trait and age-structured population from [47] to which we refer for examples, more details and full proofs.

Individuals are characterized by their ages with values in $\mathbb{R}_{+}^{d}$. Each component of this vector is an age belonging to $\mathbb{R}_{+}$, which can increase nonlinearly in time. Let $n \in \mathbb{N}^{*}$ the set of positive integers (the interpretation of $n$ is given below). We describe the population by a measure belonging to $\mathcal{M}_{P}^{n}\left(\mathbb{R}_{+}^{d}\right)$ (the set of point measures on $\mathbb{R}_{+}^{d}$ with atoms weighted by $1 / n$ ) included in $\mathcal{M}_{F}\left(\mathbb{R}_{+}^{d}\right)$ (the set of finite measures on $\mathbb{R}_{+}^{d}$ ):

$$
Z_{t}^{n}=\frac{1}{n} \sum_{i=1}^{N_{t}^{n}} \delta_{a_{i}(t)}, \quad \text { where } \quad N_{t}^{n}=n\left\langle Z_{t}^{n}, 1\right\rangle=n \int_{\mathbb{R}_{+}^{d}} Z_{t}^{n}(\mathrm{~d} a)
$$

is the number of individuals living at time $t$.

An individual of ages $a \in \mathbb{R}_{+}^{d}$ in the population $Z \in \mathcal{M}_{F}\left(\mathbb{R}_{+}^{d}\right)$ reproduces asexually, ages and dies:

1. It gives birth to a new individual of ages zero with rate $b(a) \in \mathbb{R}_{+}$.

2. Let $U: \mathbb{R}_{+}^{d} \times \mathbb{R}_{+}^{d} \rightarrow \mathbb{R}^{d_{c}}$ be an interaction kernel, $d_{c} \in \mathbb{N}^{*}$ being the number of interactions taken into account. For $a, \alpha \in \mathbb{R}_{+}^{d}, U(a, \alpha)$ models the action of an individual of ages $\alpha$ on an individual of ages $a$. The death rate of our individual is modelled by $d(a, Z U(a))$, with $d: \mathbb{R}_{+}^{d} \times \mathbb{R}^{d_{c}} \rightarrow \mathbb{R}_{+}$and where $Z U(a)=$ $\int_{\mathbb{R}_{+}^{d}} U(a, \alpha) Z(\mathrm{~d} \alpha)$

3. Our individual ages with the speed $v(a) \in \mathbb{R}_{+}^{d}$.

We introduce in Section 2 a pathwise description of our microscopic process, using Poisson point measures and the flow of an equation describing the aging phenomenon.

The parameter $n \in \mathbb{N}^{*}$ in (1.1) is related to the large population limit that will interest us in this work, and which corresponds to $n \rightarrow+\infty$. The underlying idea is to let the number of individuals grow proportionally to $n$ while their masses and the intensity of their interactions are renormalized by $1 / n$. This can be understood as a constraint in ressources: if we increase the size of the population, we have to decrease the biomass of individuals to keep the system alive. In Section 3, we prove the convergence in law in $\mathbb{D}:=\mathbb{D}\left(\mathbb{R}_{+}, \mathcal{M}_{F}\left(\mathbb{R}_{+}^{d}\right)\right)$ of 
the sequence $\left(Z^{n}\right)_{n \in \mathbb{N}^{*}}$ to the solution $\xi \in \mathcal{C}\left(\mathbb{R}_{+}, \mathcal{M}_{F}\left(\mathbb{R}_{+}^{d}\right)\right)$ of: $\forall\left(f:(a, s) \mapsto f_{s}(a)\right) \in \mathcal{C}_{b}^{1}\left(\mathbb{R}_{+}^{d} \times \mathbb{R}_{+}, \mathbb{R}_{+}\right)$,

$$
\left\langle\xi_{t}, f_{t}\right\rangle=\left\langle\xi_{0}, f_{0}\right\rangle+\int_{0}^{t} \int_{\mathbb{R}_{+}^{d}}\left[v(a) \nabla_{a} f_{s}(a)+\frac{\partial f_{s}}{\partial s}(a)+f_{s}(0) b(a)-f_{s}(a) d\left(a, \xi_{s} U(x, a)\right)\right] \xi_{s}(d a) \mathrm{d} s .
$$

This convergence result is an adaptation of results due to Fournier and Méléard [17] and Champagnat et al. [7] for populations without age structure. The densities $m(a, t)$ of the measures $\xi_{t} \in \mathcal{M}_{F}\left(\mathbb{R}_{+}^{d}\right)$, when they exist, correspond to the notion of number density, and describe the distribution in age of a population consisting in a "continuum" of individuals. We will see that absolute continuity with respect to the Lebesgue measure holds only for a scalar age $(d=1)$ and in this case, the densities solve the system: $\forall a \in \mathbb{R}_{+}, \forall t \in \mathbb{R}_{+}$,

$$
\begin{aligned}
& \frac{\partial m}{\partial t}(a, t)=-\frac{\partial}{\partial a}(v(a) m(a, t))-d\left(a, \int_{\mathbb{R}_{+}} U(a, \alpha) m(\alpha, t) \mathrm{d} \alpha\right) m(a, t) \\
& v(0) m(0, t)=\int_{\mathbb{R}_{+}} m(a, t) b(a) \mathrm{d} a, \quad m(a, 0)=m_{0}(a) .
\end{aligned}
$$

These equations generalize the PDEs introduced by McKendrick [35], Von Foerster [16] and Gurtin MacCamy [22]. This is considered in Section 3.3. Equations (1.2) and (1.3)-(1.4) are macroscopic deterministic approximations describing the ecology at the scale of the population. Individual trajectories are lost, as well as stochasticity since an averaging phenomenon occurs.

Let $T>0$, and let us consider the evolution problem on the compact time interval $[0, T]$. We use the notation $\mathbb{D}_{T}:=\mathbb{D}\left([0, T], \mathcal{M}_{F}\left(\mathbb{R}_{+}^{d}\right)\right)$. Exponential deviations are considered in Section 4 for the general model. The large deviation upper-bound is proved by establishing exponential tightness. The main difficulty lies in the fact that $\mathcal{M}_{F}\left(\mathbb{R}_{+}^{d}\right)$ is not compact. Our proof of the local lower bound relies on the use of a Riesz theorem in Orlicz spaces. This allows us to establish the links between the trajectories in the domain of the rate function and solutions of PDEs obtained by perturbing (1.2). Regularizing the perturbations and using a Girsanov theorem allow us to conclude.

These precise results on the convergence of the microscopic process to the solution of (1.2) allow us to study carefully the similarities and dissemblance between the stochastic process and its large population limit. In Section 5, from the exponential deviations of Section 4 and from the central limit theorem proved in [47], we know that on the compact time interval $[0, T]$, the microscopic process behaves like its deterministic approximation up to a small probability set. Their large time behaviors can however be radically different. We present in the following an example to illustrate this.

In the logistic age-structured population, represented by $Z \in \mathcal{M}_{F}\left(\mathbb{R}_{+}\right)$, individuals are characterized by their scalar physical age $a \in \mathbb{R}_{+}$growing with speed 1, give birth with rate $b(a)$ (continuous and upper bounded by $\bar{b}$ ) and die with rate $d(a)+\eta\langle Z, 1\rangle$. The term $d$ is the natural death rate (assumed continuous and bounded above and below by positive constants $\bar{d}$ and $\underline{d}$ ), and $\eta\langle Z, 1\rangle$ is the logistic competition term of intensity $\eta>0$. The system (1.3)-(1.4) becomes:

$$
\begin{aligned}
\frac{\partial m}{\partial t}(a, t) & =-\frac{\partial m}{\partial a}(a, t)-\left(d(a)+\eta M_{t}\right) m(a, t) \\
m(0, t) & =\int_{0}^{+\infty} b(a) m(a, t) \mathrm{d} a, \quad m(a, 0)=m_{0}(a), \quad M_{t}=\int_{0}^{+\infty} m(a, t) \mathrm{d} a .
\end{aligned}
$$

If $R_{0}:=\int_{0}^{+\infty} b(a) \mathrm{e}^{-\int_{0}^{a} d(\alpha) \mathrm{d} \alpha} \mathrm{d} a>1$, we are in a super-critical case and there exists a globally stable stationary solution to (1.5) that we denote by $\widehat{m}(a)$ (see $[6,22,51]$ ). It is interesting to notice that the microscopic process $Z^{n}$ has a different long time behaviour. It follows its deterministic approximation on compact time intervals but leaves almost surely the neighborhood of $\widehat{m}(a) d a$ to drive the population to extinction (see Fig. 1 and Props. 5.4 and 5.5 in Sect. 5). 


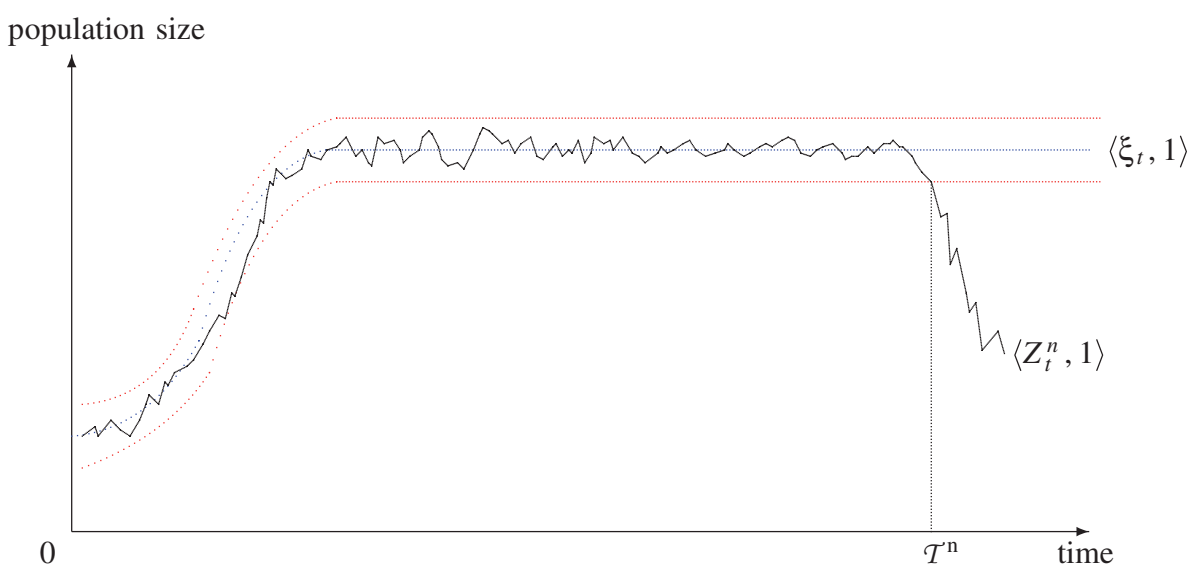

FiguRE 1. Time behaviors of the microscopic process and of its large population approximation. For sufficiently large $n$, the behaviour of the stochastic microscopic population $Z^{n}$ follows the one of its deterministic approximation $\xi$ on compact time intervals. In the long time, however, $Z^{n}$ leaves the neighborhood of the stationary stable solution of $\xi$ and the population gets extinct.

For various applications, including the studies of evolution problems for trait and age structured populations (see $[37,47])$, it is interesting to establish estimates for the time of exit from a neighborhood of the stable equilibrium of (1.5) which gives us information on the time of extinction. This can be obtained by using large deviations techniques and by adapting the results of Freidlin and Ventzell to our measure-valued processes. We show that this exit time is exponential in $n$ (Props. 5.6 and 5.11).

Notation. For two metric spaces $E$ and $F, \mathcal{C}_{b}(E, F)\left(\operatorname{resp} . \mathbb{D}(E, F), \mathcal{C}_{0}(E, \mathbb{R}), \mathcal{C}_{b}^{1}(E, F), \mathcal{C}_{K}(E, \mathbb{R}), \mathcal{B}_{b}(E, F)\right)$ is the set of continuous bounded functions from $E$ to $F$ embedded with the uniform convergence norm (resp. of càdlàg functions from $E$ to $F$ embedded with the Skorohod distance, of real continuous functions with limit 0 at infinity, of differentiable and bounded functions with bounded partial derivatives, of continuous functions with compact support, of bounded measurable functions).

The space of finite measures on $\mathbb{R}_{+}^{d}$ is denoted by $\mathcal{M}_{F}\left(\mathbb{R}_{+}^{d}\right)$. It can be embedded with the weak or vague convergence topology. By default, we will consider the weak convergence topology. We will write $\left(\mathcal{M}_{F}\left(\mathbb{R}_{+}^{d}\right), w\right)$ or $\left(\mathcal{M}_{F}\left(\mathbb{R}_{+}^{d}\right), v\right)$ to precise it when necessary.

We will consider the total variation norm and the $L^{1}$-Vaserstein distance on $\mathcal{M}_{F}\left(\mathbb{R}_{+}^{d}\right)$ :

$$
\begin{aligned}
& \forall \mu, \nu \in \mathcal{M}_{F}\left(\mathbb{R}_{+}^{d}\right),\|\nu-\mu\|_{T V}=\sup _{f \mathcal{C}_{b}\left(\mathbb{R}_{+}^{d}, \mathbb{R}\right)}\left|\int_{\mathbb{R}_{+}^{d}} f \mathrm{~d} \mu-\int_{\mathbb{R}_{+}^{d}} f \mathrm{~d} \nu\right|, \\
& \|f\|_{\infty} \leq 1 \\
& \mathcal{W}_{1}(\mu, \nu)=\inf \left\{\int_{\left(\mathbb{R}_{+}^{d}\right)^{2}}(|a-\alpha| \wedge 1) d \pi(a, \alpha)\right\}=\sup _{f \underset{f-\operatorname{Lip}\left(\mathbb{R}_{+}^{d}\right)}{\|f\|_{\infty} \leq 1}}\left|\int_{\mathbb{R}_{+}^{d}} f \mathrm{~d} \mu-\int_{\mathbb{R}_{+}^{d}} f \mathrm{~d} \nu\right|
\end{aligned}
$$

the infimum being taken on the set of measures $\pi \in \mathcal{M}_{F}\left(\left(\mathbb{R}_{+}^{d}\right)^{2}\right)$ with marginals $\mu$ and $\nu$ (see Rachev [41], Villani [49] Th. 7.12 and Rem. 7.5).

For $m \in \mathcal{M}_{F}\left(\mathbb{R}_{+}^{d}\right)$ and $f \in \mathcal{B}_{b}\left(\mathbb{R}_{+}^{d}\right)$, we write $\langle m, f\rangle$ or $\langle m, f(a)\rangle$ for $\int_{\mathbb{R}_{+}^{d}} f(a) m(d a)$.

$C$ is a constant that can change from line to line. 


\section{Microscopic PROCESS}

We now precise the individual dynamic of our model and describe the path of $Z^{n}$ by a SDE.

\subsection{Aging phenomenon}

The aging phenomenon is deterministic, and we describe it thanks to the flow of an ordinary differential equation $(O D E)$. The ages of an individual aged $a_{0} \in \mathbb{R}_{+}^{d}$ at time $t_{0} \in \mathbb{R}_{+}$satisfy:

$$
\forall t \geq t_{0}, \frac{\mathrm{d} a}{\mathrm{~d} t}=v(a(t)), \quad a\left(t_{0}\right)=a_{0},
$$

where $v(a)=\left(v_{1}(a), \cdots, v_{d}(a)\right) \in \mathbb{R}_{+}^{d}$ is the vector of aging velocities. The $i$ th component $v_{i}(a)$ of $v(a)$ is the speed of aging of the $i$ th age. Constant components correspond to ages which increase linearly in time. Non constant speeds of aging modelize ages which are measured on physiological criteria and which evolve non linearly in time.

Assumption 1. $v \in \mathcal{C}_{b}^{1}\left(\mathbb{R}_{+}^{d}, \mathbb{R}_{+}^{d}\right)$ and $\exists \bar{v}>0, \forall i \in \llbracket 1, d \rrbracket, \forall a \in \mathbb{R}_{+}^{d}, 0<v_{i}(a) \leq \bar{v}\left(1+a_{i}\right)$.

This is a technical assumption, under which the following result holds:

Proposition 2.1. Under Assumption 1:

(i) The system (2.1) admits for every $t_{0} \in \mathbb{R}_{+}, a_{0} \in \mathbb{R}_{+}^{d}$ a unique solution, defining a $\mathcal{C}^{1}$-flow:

$$
A:\left(t, t_{0}, a_{0}\right) \in \mathbb{R}_{+} \times \mathbb{R}_{+} \times \mathbb{R}_{+}^{d} \mapsto A\left(t, t_{0}, a_{0}\right) \in \mathbb{R}^{d} .
$$

Each component of this flow is increasing in $t$.

(ii) $\forall t_{1}, t_{2} \in \mathbb{R}_{+}, a \in \mathbb{R}_{+}^{d} \mapsto A\left(t_{1}, t_{2}, a\right) \in \mathbb{R}^{d}$ defines a $\mathcal{C}^{1}$-diffeomorphism from $\mathbb{R}_{+}^{d}$ on its image.

(iii) When $d=1, \forall\left(a_{0}, t_{0}\right) \in \mathbb{R}_{+}^{2}, t \in \mathbb{R}_{+} \mapsto A\left(t, t_{0}, a_{0}\right) \in \mathbb{R}$ defines a $\mathcal{C}^{1}$-diffeomorphism from $\mathbb{R}_{+}$on its image.

Proposition 2.1 is a consequence of classical ODE results (see for instance [52], Chap. 10). The following PDE (2.3), which often appears in the sequel, is solved by using the flow (2.2):

Proposition 2.2. Under Assumption 1, for $T>0$ and $\phi \in \mathcal{C}^{1}\left(\mathbb{R}_{+}^{d}, \mathbb{R}_{+}\right)$, the following transport equation with condition at time $T: \forall a \in \mathbb{R}_{+}^{d}, \forall t \in \mathbb{R}_{+}$,

$$
\frac{\partial f}{\partial t}(a, t)+v(a) \nabla_{a} f(a, t)=0, \quad f(a, T)=\phi(a),
$$

admits a unique solution $f \in \mathcal{C}^{1}\left(\mathbb{R}_{+}^{d} \times \mathbb{R}_{+}\right)$given by $\forall a \in \mathbb{R}_{+}^{d}, \forall t \in \mathbb{R}_{+}, f(a, t)=\phi(A(T, t, a))$, where $A$ is the flow defined in (2.2).

The proof is given in [15] (Chap. 3). A simple and important example is the following:

Example 2.3. For the physical age, with aging velocity 1 , the solution $(2.2)$ of equation $(2.1)$ is $A\left(t, t_{0}, a_{0}\right)=$ $a_{0}+\left(t-t_{0}\right)$ and we can easily check that $f(a, t)=\phi(a+T-t)$ solves $(2.3)$.

The other assumptions, concerning the birth and death rates are the following:

Assumption 2. We assume that $b, d$ and $U$ are continuous and that:

(i) $\exists \bar{b}>0, \forall a \in \mathbb{R}_{+}^{d},|b(a)| \leq \bar{b}$.

(ii) $\exists \bar{U}>0, \forall a, \alpha \in \mathbb{R}_{+}^{d},|U(a, \alpha)| \leq \bar{U}$.

(iii) $\exists L_{d}>0, \bar{d}>0, \forall u, v \in \mathbb{R}^{d_{c}}, \forall a \in \mathbb{R}_{+}^{d},|d(a, u)-d(a, v)| \leq L_{d}|u-v|$, and $d(a, u) \leq \bar{d}(1+|u|)$.

(iv) $\exists \underline{d} \in \mathcal{C}_{b}\left(\mathbb{R}_{+}^{d}, \mathbb{R}_{+}\right), \forall a \in \mathbb{R}_{+}^{d}, \forall u \in \mathbb{R}^{d_{c}}, d(a, u) \geq \underline{d}(a)$, and $\forall t_{0} \in \mathbb{R}_{+}, \forall a_{0} \in \mathbb{R}_{+}^{d}$,

$$
\int_{t_{0}}^{+\infty} \underline{d}\left(A\left(t, t_{0}, a_{0}\right)\right) \mathrm{d} t=+\infty .
$$


The function $\underline{d}$ in (iv) can be interpreted as a natural death rate, and Point (iv) can be linked to the survival probability, which is the probability that an individual of ages $a_{0}$ at time $t_{0}$ is still alive at time $t$ :

$$
\Pi\left(t_{0}, a_{0}, t\right)=\mathbb{E}\left[\exp \left(-\int_{t_{0}}^{t} d\left(A\left(u, t_{0}, a_{0}\right), Z_{u} U\left(x, A\left(u, t_{0}, a_{0}\right)\right)\right) \mathrm{d} u\right)\right] .
$$

Under Point (iv), $\lim _{t \rightarrow+\infty} \Pi\left(t_{0}, a_{0}, t\right)=0$.

\subsection{Stochastic differential equation}

We introduce a SDE driven by a Poisson point measure, for which existence and uniqueness of the solution are stated. The solution is a Markov process, with a generator that corresponds to the dynamics described previously. We follow in this the approach of $[7,8,17]$.

Let us introduce the following map $\pi=\left(\pi_{1}, \cdots, \pi_{N}, \cdots\right)$ from $\bigcup_{n \in \mathbb{N}^{*}} \mathcal{M}_{P}^{n}\left(\mathbb{R}_{+}^{d}\right)$ in $\left(\mathbb{R}_{+}^{d}\right)^{\mathbb{N}}$ that will be useful to extract a particular individual from the population where the particles are ranked in the lexicographical order of $\mathbb{R}_{+}^{d}: \forall n, N \in \mathbb{N}^{*}$,

$$
\pi\left(\frac{1}{n} \sum_{i=1}^{N} \delta_{a_{i}}\right)=\left(a_{1}, \cdots, a_{N}, 0, \cdots, 0, \cdots\right)
$$

Definition 2.4. On the probability space $(\Omega, \mathcal{F}, \mathbb{P})$, we consider:

1. a sequence $\left(Z_{0}^{n}\right)_{n \in \mathbb{N}^{*}}$ of random variables with $\forall n \in \mathbb{N}^{*}, Z_{0}^{n} \in \mathcal{M}_{P}^{n}\left(\mathbb{R}_{+}^{d}\right)$ and $\sup _{n \in \mathbb{N}^{*}} \mathbb{E}\left(\left\langle Z_{0}^{n}, 1\right\rangle\right)<+\infty$;

2. a Poisson point measure $Q(d s, d i, d \theta)$ on $\mathbb{R}_{+} \times \mathcal{E}$ where $\mathcal{E}:=\mathbb{N}^{*} \times \mathbb{R}_{+}$of intensity $d s \otimes n(d i) \otimes d \theta$ (where $d s$ and $d \theta$ are Lebesgue measures on $\mathbb{R}_{+}$and $n(d i)$ is the counting measure on $\left.\mathbb{N}^{*}\right)$ independent from $\left(Z_{0}^{n}\right)_{n \in \mathbb{N}^{*}}$. We denote by $\left(\mathcal{F}_{t}\right)_{t \in \mathbb{R}_{+}}$the canonical filtration associated with $Z_{0}^{n}$ and $Q$. For $t \in \mathbb{R}_{+}, Z_{t}^{n}$ is described by:

$$
\begin{aligned}
Z_{t}^{n}= & \frac{1}{n} \sum_{i=1}^{N_{0}^{n}} \delta_{A\left(t, 0, \pi_{i}\left(Z_{0}^{n}\right)\right)}+\frac{1}{n} \int_{0}^{t} \int_{\mathcal{E}} \mathbf{1}_{\left\{i \leq N_{s_{-}}^{n}\right\}}\left[\delta_{A(t, s, 0)} \mathbf{1}_{\left\{0 \leq \theta<m_{1}\left(s, Z_{s_{-}}^{n}, i\right)\right\}}\right. \\
& \left.-\delta_{A\left(t, s, \pi_{i}\left(Z_{s_{-}}^{n}\right)\right)} \mathbf{1}_{\left\{m_{1}\left(s, Z_{s_{-}}^{n}, i\right) \leq \theta<m_{2}\left(s, Z_{s_{-}}^{n}, i\right)\right\}}\right] Q(d s, d i, d \theta),
\end{aligned}
$$

where $N_{s}^{n}$ is defined in (1.1), where $A$ is the flow defined in (2.2), and where:

$$
m_{1}\left(s, Z_{s_{-}}^{n}, i\right)=b\left(\pi_{i}\left(Z_{s_{-}}^{n}\right)\right), \quad m_{2}\left(s, Z_{s_{-}}^{n}, i\right)=m_{1}\left(s, Z_{s_{-}}^{n}, i\right)+d\left(\pi_{i}\left(Z_{s_{-}}^{n}\right), Z_{s_{-}}^{n} U\left(\pi_{i}\left(Z_{s_{-}}^{n}\right)\right)\right) .
$$

Let $T_{0}^{n}=0$. Assume that the size $N_{t}^{n}$ of the population at time $t \in \mathbb{R}_{+}$is finite. Under Assumptions 2, there exists a positive constant $\bar{C}$ (say $\bar{b}+\bar{d}$ ) such that the global jump rate at time $t \in \mathbb{R}_{+}$is upper bounded by $\bar{C} N_{t_{-}}^{n}\left(1+N_{t_{-}}^{n}\right)$, which is finite. Hence, it is possible to define the sequence of jump times $\left(T_{k}^{n}\right)_{k \in \mathbb{N}^{*}}$ of $Z^{n}$ almost surely. Since it forms an increasing sequence, $T_{\infty}^{n}:=\lim _{k \rightarrow+\infty} T_{k}^{n}$ is well defined. It is proved in [47] (Sects. 2.2, 2.3 and 3.1) that:

Theorem 2.5 (existence and uniqueness of the solutions of (2.5)). Under Assumptions 1, 2 and Point 1 of Definition 2.4, $T_{\infty}^{n}=+\infty \mathbb{P}$-a.s. and SDE (2.5) admits for every $n \in \mathbb{N}^{*}$ a unique pathwise solution $\left(Z_{t}^{n}\right)_{t \in \mathbb{R}_{+}} \in \mathbb{D}$.

Since $N_{t}^{n}$ and $\left\langle Z_{t}^{n},|a|\right\rangle$ are unbounded, we introduce the following stopping times: let $N \in \mathbb{R}_{+}^{*}$,

$$
\tau_{N}^{n}=\inf \left\{t \geq 0, N_{t}^{n} \geq N\right\}, \quad \zeta_{N}^{n}=\inf \left\{t \geq 0, N_{t}^{n} \geq N \text { or }\left\langle Z_{t}^{n},|a|\right\rangle \geq N\right\} .
$$




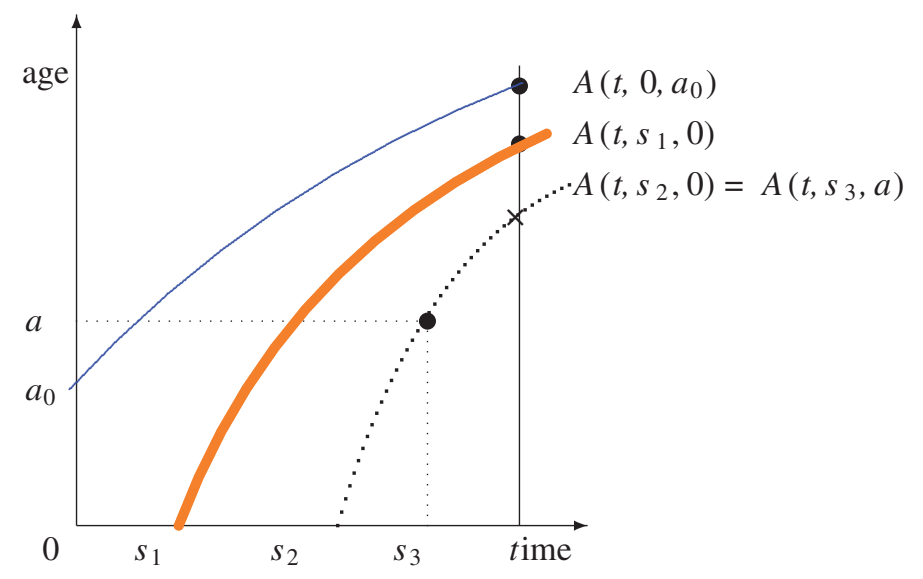

FIgURE 2. The interpretation is that the state of the population at time $t$ is obtained by considering the initial particles with their ages at times $t$ (for example, $A\left(t, 0, a_{0}\right)$ for the blue thin-line particle), by adding the particles born between $s=0$ and $s=t$ with their age at time $t\left(A\left(t, s_{1}, 0\right)\right.$ and $A\left(t, s_{2}, 0\right)$ for the particles in thick orange and black dot lines) and by suppressing the particles which have died before time $t\left(A\left(t, s_{3}, a\right)\right.$ for the particle in black dot-line).

\subsection{Moment and martingale properties}

We give some moment and martingale properties that will be useful in the sequel.

Lemma 2.6. Let $F \in \mathcal{C}^{1}(\mathbb{R}, \mathbb{R}),\left(f:(a, s) \mapsto f_{s}(a)\right) \in \mathcal{C}_{b}^{1}\left(\mathbb{R}_{+}^{d} \times \mathbb{R}_{+}, \mathbb{R}\right) . \forall t \in \mathbb{R}_{+}, \forall n \in \mathbb{N}^{*}$,

$$
\begin{aligned}
F\left(\left\langle Z_{t}^{n}, f_{t}\right\rangle\right)= & F\left(\left\langle Z_{0}^{n}, f_{0}\right\rangle\right)+\int_{0}^{t}\left\langle Z_{s}^{n}, v \nabla_{a} f_{s}+\frac{\partial f_{s}}{\partial s}\right\rangle F^{\prime}\left(\left\langle Z_{s}^{n}, f_{s}\right\rangle\right) \mathrm{d} s \\
& +\int_{0}^{t} \int_{\mathcal{E}} \mathbf{1}_{\left\{i \leq N_{t}^{n}\right\}}\left[\left(F\left(\left\langle Z_{s_{-}}^{n}, f_{s}\right\rangle+\frac{f_{s}(0)}{n}\right)-F\left(\left\langle Z_{s_{-}}^{n}, f_{s}\right\rangle\right)\right) \mathbf{1}_{\left\{0 \leq \theta<m_{1}\left(s, Z_{s_{-}}^{n}, i\right)\right\}}\right. \\
& \left.+\left(F\left(\left\langle Z_{s_{-}}^{n}, f_{s}\right\rangle-\frac{f_{s}\left(\pi_{i}\left(Z_{s_{-}}^{n}\right)\right)}{n}\right)-F\left(\left\langle Z_{s_{-}}^{n}, f_{s}\right\rangle\right)\right) \mathbf{1}_{\left\{m_{1}\left(s_{2}, Z_{s_{-}}^{n}, i\right) \leq \theta<m_{2}\left(s, Z_{s_{-}}^{n}, i\right)\right\}}\right] Q(d s, d i, d \theta)
\end{aligned}
$$

Proof. Integrating $f_{t}(a)$ with respect to (2.5) gives:

$$
\begin{aligned}
\left\langle Z_{t}^{n}, f_{t}\right\rangle= & \frac{1}{n} \sum_{i=1}^{N_{0}^{n}} f_{t}\left(A\left(t, 0, \pi_{i}\left(Z_{0}^{n}\right)\right)\right)+\frac{1}{n} \int_{0}^{t} \int_{\mathcal{E}} \mathbf{1}_{\left\{i \leq N_{s_{-}}^{n}\right\}}\left[f_{t}(A(t, s, 0)) \mathbf{1}_{\left\{0 \leq \theta<m_{1}\left(s, Z_{s_{-}}^{n}, i\right)\right\}}\right. \\
& \left.-f_{t}\left(A\left(t, s, \pi_{i}\left(Z_{s_{-}}^{n}\right)\right)\right) \mathbf{1}_{\left\{m_{1}\left(s, Z_{s_{-}}^{n}, i\right) \leq \theta<m_{2}\left(s, Z_{s_{-}}^{n}, i\right)\right\}}\right] Q(d s, d i, d \theta) .
\end{aligned}
$$

Since $\forall a \in \mathbb{R}_{+}^{d}, \forall 0 \leq s \leq t$,

$$
f_{t}(A(t, s, a))=f_{s}(a)+\int_{s}^{t}\left(\frac{\partial f_{u}}{\partial u}(A(u, s, a))+v(A(u, s, a)) \nabla_{a} f_{u}(A(u, s, a))\right) \mathrm{d} u
$$


we deduce:

$$
\begin{aligned}
\left\langle Z_{t}^{n}, f_{t}\right\rangle= & \frac{1}{n} \sum_{i=1}^{N_{0}^{n}} f_{0}\left(\pi_{i}\left(Z_{0}^{n}\right)\right)+\frac{1}{n} \int_{0}^{t} \int_{\mathcal{E}} \mathbf{1}_{\left\{i \leq N_{s_{-}}^{n}\right\}}\left[f_{s}(0) \mathbf{1}_{\left\{0 \leq \theta<m_{1}\left(s, Z_{s_{-}}^{n}, i\right)\right\}}\right. \\
& \left.-f_{s}\left(\pi_{i}\left(Z_{s_{-}}^{n}\right)\right) \mathbf{1}_{\left\{m_{1}\left(s, Z_{s_{-}}^{n}, i\right) \leq \theta<m_{2}\left(s, Z_{s_{-}}^{n}, i\right)\right\}}\right] Q(d s, d i, d \theta)+T_{1}+T_{2}+T_{3},
\end{aligned}
$$

where:

$$
\begin{aligned}
T_{1}= & \frac{1}{n} \int_{0}^{t} \sum_{i=1}^{N_{0}^{n}}\left(\frac{\partial f_{s}}{\partial s}\left(A\left(s, 0, \pi_{i}\left(Z_{0}^{n}\right)\right)\right)+v\left(A\left(s, 0, \pi_{i}\left(Z_{0}^{n}\right)\right)\right) \nabla_{a} f_{s}\left(A\left(s, 0, \pi_{i}\left(Z_{0}^{n}\right)\right)\right)\right) \mathrm{d} s \\
T_{2}= & \frac{1}{n} \int_{0}^{t} \int_{\mathcal{E}} \mathbf{1}_{\left\{i \leq N_{s_{-}}^{n}\right\}} \int_{s}^{t}\left(\frac{\partial f_{u}}{\partial u}(A(u, s, 0))+v(A(u, s, 0)) \nabla_{a} f_{u}(A(u, s, 0))\right) \mathrm{d} u \mathbf{1}_{\left\{0 \leq \theta<m_{1}\left(s, Z_{s_{-}}^{n}, i\right)\right\}} Q(d s, d i, d \theta) \\
T_{3}= & \frac{1}{n} \int_{0}^{t} \int_{\mathcal{E}} \mathbf{1}_{\left\{i \leq N_{s_{-}}^{n}\right\}} \int_{s}^{t}\left(\frac{\partial f_{u}}{\partial u}\left(A\left(u, s, \pi_{i}\left(Z_{s_{-}}^{n}\right)\right)\right)+v\left(A\left(u, s, \pi_{i}\left(Z_{s_{-}}^{n}\right)\right)\right) \nabla_{a} f_{u}\left(A\left(u, s, \pi_{i}\left(Z_{s_{-}}^{n}\right)\right)\right)\right) \mathrm{d} u \\
& \mathbf{1}_{\left\{m_{2}\left(s, Z_{s_{-}}^{n}, i\right) \leq \theta<m_{3}\left(s, Z_{s_{-}}^{n}, i\right)\right\}} Q(d s, d i, d \theta) .
\end{aligned}
$$

It is possible to apply Fubini's theorem to $T_{2}$ and $T_{3}$ and by (2.5) we recognize:

$$
T_{1}+T_{2}+T_{3}=\int_{0}^{t}\left[\int_{\mathbb{R}_{+}^{d}}\left(\frac{\partial f_{u}}{\partial u}(a)+v(a) \nabla_{a} f_{u}(a)\right) Z_{u}^{n}(d a)\right] \mathrm{d} u .
$$

From (2.8), (2.9), and applying Itô's formula with jump terms, we obtain (2.7).

Proposition 2.7. Under Assumptions 1 and 2,

(i) if $\exists q \geq 1, \sup _{n \in \mathbb{N}^{*}} \mathbb{E}\left(\left\langle Z_{0}^{n}, 1\right\rangle^{q}\right)<+\infty$, then:

$$
\forall n \in \mathbb{N}^{*}, \lim _{N \rightarrow+\infty} \tau_{N}^{n}=+\infty, \mathbb{P}-\text { a.s., } \quad \text { and } \quad \forall T>0, \sup _{n \in \mathbb{N}^{*}} \mathbb{E}\left(\sup _{t \in[0, T]}\left\langle Z_{t}^{n}, 1\right\rangle^{q}\right)<+\infty ;
$$

(ii) if $\exists q \geq 1, m \geq 1, \sup _{n \in \mathbb{N}^{*}} \mathbb{E}\left(\left\langle Z_{0}^{n}, 1\right\rangle^{m}\right)<+\infty$ and $\sup _{n \in \mathbb{N}^{*}} \mathbb{E}\left(\left\langle Z_{0}^{n},|a|^{q}\right\rangle^{m}\right)<+\infty$, then:

$$
\forall n \in \mathbb{N}^{*}, \lim _{N \rightarrow+\infty} \zeta_{N}^{n}=+\infty, \mathbb{P}-\text { a.s. }, \quad \text { and } \quad \forall T>0, \sup _{n \in \mathbb{N}^{*}} \mathbb{E}\left(\sup _{t \in[0, T]}\left\langle Z_{t}^{n},|a|^{q}\right\rangle^{m}\right)<+\infty ;
$$

(iii) let $n \in \mathbb{N}^{*}$. If $\mathbb{E}\left(\left\langle Z_{0}^{n}, 1\right\rangle^{2}\right)<+\infty$ and $\mathbb{E}\left(\left\langle Z_{0}^{n},|a|\right\rangle\right)<+\infty$ then: $\forall f \in \mathcal{C}_{b}^{1}\left(\mathbb{R}_{+}^{d} \times \mathbb{R}_{+}, \mathbb{R}\right)$,

$$
\begin{aligned}
M_{t}^{n, f}= & \left\langle Z_{t}^{n}, f_{t}\right\rangle-\left\langle Z_{0}^{n}, f_{0}\right\rangle \\
& -\int_{0}^{t} \int_{\mathbb{R}_{+}^{d}}\left[v(a) \nabla_{a} f_{s}(a)+\frac{\partial f_{s}}{\partial s}(a)+f_{s}(0) b(a)-f_{s}(a) d\left(a, Z_{s}^{n} U(a)\right)\right] Z_{s}^{n}(d a) \mathrm{d} s,
\end{aligned}
$$

is a square integrable càdlàg martingale starting from 0, with predictable quadratic variation:

$$
\left\langle M^{n, f}\right\rangle_{t}=\frac{1}{n} \int_{0}^{t} \int_{\mathbb{R}_{+}^{d}}\left[f_{s}^{2}(0) b(a)+f_{s}^{2}(a) d\left(a, Z_{s}^{n} U(a)\right)\right] Z_{s}^{n}(d a) \mathrm{d} s .
$$


Proof. Point (i) is a direct adaptation of the proof of Lemma 5.2 in [17]. Let us consider Point (ii). Let $N>0$, $n \in \mathbb{N}^{*}$ and $\zeta_{N}^{n}$ be the stopping time introduced in (2.6). For this proof, we will consider the norm 1 on $\mathbb{R}^{d}$ : $\forall a=\left(a_{1}, \cdots a_{d}\right) \in \mathbb{R}_{+}^{d},|a|=\sum_{i=1}^{d} a_{i}$. This choice is made since it simplifies the calculations. It is not restrictive since the norms in $\mathbb{R}^{d}$ are equivalent. The function $f: a \in \mathbb{R}_{+}^{d} \mapsto|a|^{q}=\left(\sum_{i=1}^{d} a_{i}\right)^{q} \in \mathbb{R}_{+}$is differentiable and $\forall i \in \llbracket 1, d \rrbracket, \partial f / \partial a_{i}=q|a|^{q-1}$. The map $F: x \in \mathbb{R}_{+} \mapsto x^{m} \in \mathbb{R}_{+}$is also differentiable and $F^{\prime}(x)=m x^{m-1}$. Applying Lemma 2.6 and neglecting the non positive terms gives:

$$
\mathbb{E}\left(\sup _{u \in\left[0, t \wedge \zeta_{N}^{n}\right]}\left\langle Z_{u}^{n},|a|^{q}\right\rangle^{m}\right) \leq \mathbb{E}\left(\left\langle Z_{0}^{n},|a|^{q}\right\rangle^{m}\right)+m q \bar{v} \mathbb{E}\left(\int_{0}^{t \wedge \zeta_{N}^{n}}\left\langle Z_{s}^{n},|a|^{q-1}(d+|a|)\right\rangle\left\langle Z_{s}^{n},|a|^{q}\right\rangle^{m-1} \mathrm{~d} s\right),
$$

by using the fact that $0 \leq \sum_{i=1}^{d} v_{i}(a) \leq \bar{v}(d+|a|)$ (Assumption 1). Since:

$$
\left\langle Z_{s}^{n},|a|^{q-1}\right\rangle \leq\left\langle Z_{s}^{n},(|a| \vee 1)^{q-1}\right\rangle \leq\left\langle Z_{s}^{n},(|a| \vee 1)^{q}\right\rangle \leq\left\langle Z_{s}^{n}, 1\right\rangle+\left\langle Z_{s}^{n},|a|^{q}\right\rangle
$$

we have:

$$
\left\langle Z_{s}^{n},|a|^{q-1}\right\rangle\left\langle Z_{s}^{n},|a|^{q}\right\rangle^{m-1} \leq\left\langle Z_{s}^{n}, 1\right\rangle\left\langle Z_{s}^{n},|a|^{q}\right\rangle^{m-1}+\left\langle Z_{s}^{n},|a|^{q}\right\rangle^{m} \leq\left\langle Z_{s}^{n}, 1\right\rangle^{m}+2\left\langle Z_{s}^{n},|a|^{q}\right\rangle^{m}
$$

and hence by the moment assumptions, (2.10) and by the Fubini's theorem:

$$
\begin{aligned}
\mathbb{E}\left(\sup _{u \in\left[0, t \wedge \zeta_{N}^{n}\right]}\left\langle Z_{u}^{n},|a|^{q}\right\rangle^{m}\right) \leq & \mathbb{E}\left(\left\langle Z_{0}^{n},|a|^{q}\right\rangle^{m}\right)+m q \bar{v} d T \mathbb{E}\left(\sup _{u \in\left[0, T \wedge \zeta_{N}^{n}\right]}\left\langle Z_{u}^{n}, 1\right\rangle^{m}\right) \\
& +m q \bar{v}(2 d+1) \int_{0}^{t} \mathbb{E}\left(\sup _{u \in\left[0, s \wedge \zeta_{N}^{n}\right]}\left\langle Z_{u}^{n},|a|^{q}\right\rangle^{m}\right) \mathrm{d} s \\
\leq & \left(\sup _{n \in \mathbb{N}^{*}} \mathbb{E}\left(\left\langle Z_{0}^{n},|a|^{q}\right\rangle^{m}\right)+m q \bar{v} d T C(m, T)\right) \mathrm{e}^{m q \bar{v}(2 d+1) T}=: D(q, T),
\end{aligned}
$$

by Gronwall's lemma, and where $C(m, T)$ is an upper bound of $\sup _{n \in \mathbb{N}^{*}} \mathbb{E}\left(\sup _{t \in[0, T]}\left\langle Z_{t}^{n}, 1\right\rangle^{m}\right)$. As $D(q, T)$ does not depend on $N$, we deduce that:

$$
\lim _{N \rightarrow+\infty} \zeta_{N}^{n}=+\infty, \mathbb{P}-\text { a.s. }
$$

Assume indeed that (2.16) is not satisfied. For $q=1$, there exists $M>0$ and $A_{M} \subset \Omega$ with $\mathbb{P}\left(A_{M}\right)>0$ such that $\forall \omega \in A_{M}, \lim _{N \rightarrow+\infty} \zeta_{N}^{n}(\omega)<M$. Then, for $T>M, \mathbb{E}\left(\sup _{t \in\left[0, T \wedge \zeta_{N}^{n}\right]}\left\langle Z_{t}^{n},|a|\right\rangle^{m}\right) \geq \mathbb{P}\left(A_{M}\right) N^{m}$, which can not be upper bounded independently of $N$. Hence, (2.16) is satisfied. Letting $N$ tend to infinity in (2.15) gives (2.11) by Fatou's lemma.

Point (iii) is obtained by using the compensated Poisson point measure $\widetilde{Q}$ of $Q$. We have:

$$
M_{t}^{n, f}=-\frac{1}{n} \int_{0}^{t} \int_{\mathcal{E}} \mathbf{1}_{\left\{i \leq N_{s_{-}}^{n}\right\}}\left(f_{s}(0) \mathbf{1}_{\left\{0 \leq \theta<m_{1}\left(s, Z_{s_{-}}^{n}, i\right)\right\}}-f_{s}\left(\pi_{i}\left(Z_{s_{-}}^{n}\right)\right) \mathbf{1}_{\left\{m_{1}\left(s, Z_{s_{-}}^{n}, i\right) \leq \theta \leq m_{2}\left(s, Z_{s_{-}}^{n}, i\right)\right\}}\right) \widetilde{Q}(d s, d i, d \theta) .
$$

Showing that it is a square integrable martingale and computing its quadratic variation is standard (see [47]). 
The infinitesimal generator of (2.5) corresponds to the description of the introduction:

Theorem 2.8. For $n \in \mathbb{N}^{*}, Z^{n} \in \mathbb{D}$ is a Markov process of infinitesimal generator defined by: $\forall f \in \mathcal{C}_{b}^{1}\left(\mathbb{R}_{+}^{d}, \mathbb{R}\right)$, $\forall F \in \mathcal{C}_{b}^{1}(\mathbb{R}, \mathbb{R})$,

$$
\begin{aligned}
& L F_{f}\left(Z_{0}^{n}\right)=\left.\frac{\partial}{\partial t} \mathbb{E}\left(F_{f}\left(Z_{t}^{n}\right)\right)\right|_{t=0}=\int_{\mathbb{R}_{+}^{d}} v(a) \nabla_{a} f(a) F^{\prime}\left(\left\langle Z_{0}^{n}, f\right\rangle\right) Z_{0}^{n}(d a) \\
& +n \int_{\mathbb{R}_{+}^{d}}\left[\left(F_{f}\left(Z_{0}^{n}+\frac{1}{n} \delta_{0}\right)-F_{f}\left(Z_{0}^{n}\right)\right) b(a) Z_{0}^{n}(d a)+\left(F_{f}\left(Z_{0}^{n}-\frac{1}{n} \delta_{a}\right)-F_{f}\left(Z_{0}^{n}\right)\right) d\left(a, Z_{0}^{n} U(a)\right)\right] Z_{0}^{n}(d a),
\end{aligned}
$$

where $\forall Z \in \mathcal{M}_{F}\left(\mathbb{R}_{+}^{d}\right), F_{f}(Z)=F(\langle Z, f\rangle)$.

Proof. The proof consists in proving that we can take the derivative under the expectation (see [47]).

We conclude this part with the consideration of exponential moments, which will be useful in the section dealing with exponential deviations.

Assumption 3. We assume that:

1. $\forall \lambda>0, \sup _{n \in \mathbb{N}^{*}} \mathbb{E}\left(\mathrm{e}^{\lambda\left\langle Z_{0}^{n}, 1\right\rangle}\right)<+\infty$, and $\left.\exists \eta \in\right] 0,1\left[\sup _{n \in \mathbb{N}^{*}} \mathbb{E}\left(\left[\int_{\mathbb{R}_{+}^{d}}|a| Z_{0}^{n}(d a)\right]^{1+\eta}\right)<+\infty\right.$.

2. $\exists i_{0} \in \llbracket 1, d_{c} \rrbracket, \exists n_{0} \in \mathbb{N}^{*}, \exists U_{0} \in \mathbb{R}_{+}^{*}, \forall n \geq n_{0}, \forall u \in \mathbb{R}^{d_{c}}, \forall a \in \mathbb{R}_{+}^{d}, \forall \lambda>0$,

$$
\left[u_{i_{0}} \geq U_{0}\right] \Rightarrow\left[\left(\mathrm{e}^{\lambda / n}-1\right) b(a)+\left(\mathrm{e}^{-\lambda / n}-1\right) d(a, u)<0\right],
$$

( $d_{c}$ is the number of interactions).

3. $\exists \underline{U}>0, \forall a, \alpha \in \mathbb{R}_{+}^{d}, U_{i_{0}}(a, \alpha)>\underline{U}$, for the index $i_{0}$ of Point 2.

Since $\forall a \in \mathbb{R}_{+}^{d}, b(a) \leq \bar{b}$, Point 2 is for instance satisfied if $\lim _{|u| \rightarrow+\infty} d(a, u)=+\infty$ (this happens for the logistic model).

Proposition 2.9. Under Assumptions 1, 2, 3:

(i) $\forall \lambda>0, \forall t \in[0, T]$,

$$
\sup _{n \in \mathbb{N}^{*}} \mathbb{E}\left(\mathrm{e}^{\lambda\left\langle Z_{t}^{n}, 1\right\rangle}\right) \leq \sup _{n \in \mathbb{N}^{*}} \mathbb{E}\left(\mathrm{e}^{\lambda\left\langle Z_{0}^{n}, 1\right\rangle}\right)+\lambda \bar{b} M T \mathrm{e}^{\lambda M}<+\infty
$$

where $M:=U_{0} / \underline{U}$ with the constants $U_{0}$ and $\underline{U}$ of Assumption 3.

(ii) Let $\rho(x)=\mathrm{e}^{x}-x-1$. We consider the martingale $\left(M_{t}^{n, f}\right)_{t \in \mathbb{R}_{+}}$defined in (2.12). Then, the process defined for $t \in[0, T], n \in \mathbb{N}^{*}$ and $f \in \mathcal{C}_{b}\left(\mathbb{R}_{+}^{d} \times[0, T], \mathbb{R}\right)$ by $\Lambda_{t}^{n, f}=\mathrm{e}^{M_{t}^{n, f}-\Xi_{t}^{n, f}}$,

with: $\quad \Xi_{t}^{n, f}=\int_{0}^{t} \int_{\mathbb{R}_{+}^{d}} n\left[\rho\left(\frac{f(0, s)}{n}\right) b(a)-\rho\left(-\frac{f(a, s)}{n}\right) d\left(a, Z_{s}^{n} U(a)\right)\right] Z_{s}^{n}(d a) \mathrm{d} s$,

is a martingale.

Sketch of proof. We refer to [47] for a complete proof. For Point (i), the idea is that under Assumptions 3, the drift of the semi-martingale $\left(\mathrm{e}^{\lambda\left\langle Z_{\left.t \wedge \tau_{N}^{n}, 1\right\rangle}^{n}\right.}\right)_{t \in[0, T]}$ is non-negative only for measures that have a mass bounded by $M$. For Point (ii), applying Itô's formula to $\Lambda_{t}^{n, f}$ localized by the stopping time $\tau_{N}^{n}(2.6)$ yields that $\Lambda^{n, f}$ is a local martingale. From Assumptions 3 and Point (i) of Proposition 3.2, $\lim _{N \rightarrow+\infty} \tau_{N}^{n}=+\infty \mathbb{P}$-a.s. Using Fatou's lemma, we show that $\Lambda^{n, f}$ is a real martingale. 


\section{Convergence to the weak measure solution of a PDE in the large POPULATION LIMIT}

We study the limit of $\left(Z^{n}\right)_{n \in \mathbb{N}^{*}}$ when $n \rightarrow+\infty$, under the following assumptions:

Assumption 4. We assume that $\left(Z_{0}^{n}\right)_{n \in \mathbb{N}^{*}}$ converges in law in $\left(\mathcal{M}_{F}\left(\mathbb{R}_{+}^{d}\right), w\right)$ to the deterministic measure $\xi_{0} \in \mathcal{M}_{F}\left(\mathbb{R}_{+}^{d}\right)$ and that the following moment conditions are satisfied:

$$
\exists \eta \in] 0,1\left[, \quad \sup _{n \in \mathbb{N}^{*}} \mathbb{E}\left(\left\langle Z_{0}^{n}, 1\right\rangle^{2+\eta}\right)<+\infty, \quad \text { and } \quad \sup _{n \in \mathbb{N}^{*}} \mathbb{E}\left(\left\langle Z_{0}^{n},|a|\right\rangle^{1+\eta}\right)<+\infty\right.
$$

In this section, we prove the convergence of the sequence $\left(Z^{n}\right)_{n \in \mathbb{N}^{*}}$ to the solution of (1.2) by a tightnessuniqueness argument.

\subsection{Tightness of the sequence $\left(Z^{n}\right)_{n \in \mathbb{N}^{*}}$}

Proposition 3.1. Under Assumptions 1, 2, 4, $\left(Z^{n}\right)_{n \in \mathbb{N}^{*}}$ is tight on $\mathbb{D}\left(\mathbb{R}_{+},\left(\mathcal{M}_{F}\left(\mathbb{R}_{+}^{d}\right)\right.\right.$, w)). Its limit values are continuous measure-valued processes satisfying (1.2).

Proof. The proof is inspired by the proof of Theorem 5.3 in [17], and a detailed proof stands in [47], Theorem 3.2.2. We begin with establishing the tightness of the laws of $\left(Z^{n}\right)_{n \in \mathbb{N}^{*}}$ considered as processes of $\mathbb{D}\left(\mathbb{R}_{+},\left(\mathcal{M}_{F}\left(\mathbb{R}_{+}^{d}\right), v\right)\right)$, where $\mathcal{M}_{F}\left(\mathbb{R}_{+}^{d}\right)$ is embedded with the topology of vague convergence. For this purpose, we use a criterium due to Roelly [43], Th. 2.1): it is sufficient to prove that for $f \in \mathcal{C}_{b}^{1}\left(\mathbb{R}_{+}^{d}, \mathbb{R}\right) \cap \mathcal{C}_{0}\left(\mathbb{R}_{+}^{d}, \mathbb{R}\right)$, which is dense in $\mathcal{C}_{0}\left(\mathbb{R}_{+}^{d}, \mathbb{R}\right)$, the sequence $\left(Z^{n, f}=\left\langle Z^{n}, f\right\rangle\right)_{n \in \mathbb{N}^{*}}$ is tight in $\mathbb{D}\left(\mathbb{R}_{+}, \mathbb{R}\right)$. Thanks to Proposition 2.7 and Assumptions 4, we can prove that Aldous and Rebolledo criteria [1,29] are satisfied, which is a sufficient condition for $\left(Z^{n, f}\right)_{n \in \mathbb{N} *}$ to be tight.

Notice that the particular choice of $f \equiv 1$ implies the tightness of the sequence $\left(\left\langle Z^{n}, 1\right\rangle\right)_{n \in \mathbb{N}^{*}}$ in $\mathbb{D}_{\left(\mathbb{R}_{+}, \mathbb{R}_{+}\right)}$ with the same arguments.

By Prohorov theorem, it is possible to extract from $\left(Z^{n}\right)_{n \in \mathbb{N}^{*}}$ a subsequence $\left(Z^{\phi(n)}\right)_{n \in \mathbb{N}^{*}}$ that converges in law in $\mathbb{D}\left(\mathbb{R}_{+},\left(\mathcal{M}_{F}\left(\mathbb{R}_{+}^{d}\right), v\right)\right)$ to a limiting process $Z$. Since we also proved the tightness of $\left(\left\langle Z^{n}, 1\right\rangle\right)_{n \in \mathbb{N}^{*}}$, it is possible to choose this subsequence such that $\left(\left\langle Z^{\phi(n)}, 1\right\rangle\right)_{n \in \mathbb{N}^{*}}$ converges in law to $\langle Z, 1\rangle$ in $\mathbb{D}\left(\mathbb{R}_{+}, \mathbb{R}_{+}\right)$. By construction, the jumps of $\left(Z^{n}\right)_{n \in \mathbb{N}^{*}}$ are of order $1 / n$ which entails that the limit process $Z$ is almost surely continuous. Using Theorem 3 in Méléard and Roelly [36], $\left(Z^{\phi(n)}\right)_{n \in \mathbb{N}^{*}}$ also converges in law in $\mathbb{D}\left(\mathbb{R}_{+},\left(\mathcal{M}_{F}\left(\mathbb{R}_{+}^{d}\right), w\right)\right)$, where $\mathcal{M}_{F}\left(\mathbb{R}_{+}^{d}\right)$ is embedded with the topology of weak convergence. Applying Prohorov theorem again, we deduce that the sequence $\left(Z^{n}\right)_{n \in \mathbb{N}^{*}}$ is tight in $\mathbb{D}\left(\mathbb{R}_{+},\left(\mathcal{M}_{F}\left(\mathbb{R}_{+}^{d}\right), w\right)\right)$.

To identify the limit, we have to prove that $\mathbb{P}_{\text {-a.s. }}, \forall\left[f:(a, t) \mapsto f_{t}(a)\right] \in \mathcal{C}_{b}^{1}\left(\mathbb{R}_{+}^{d} \times \mathbb{R}_{+}, \mathbb{R}^{\prime}\right), \forall t \in \mathbb{R}_{+}$, $\Psi_{t}(Z)=0$, where:

$$
\Psi_{t}(Z)=\left\langle Z_{t}, f_{t}\right\rangle-\left\langle Z_{0}, f_{0}\right\rangle-\int_{0}^{t} \int_{\mathbb{R}_{+}^{d}}\left[v(a) \nabla_{a} f_{s}(a)+\frac{\partial f_{s}}{\partial s}(a)+f(0, s) b(a)-f(a, s) d\left(a, Z_{s} U(a)\right)\right] Z_{s}(d a) \mathrm{d} s
$$

This is obtained by a direct adaptation of the proof of $[17,47]$.

In order to prove that the whole sequence $\left(Z^{n}\right)_{n \in \mathbb{N}^{*}}$ converges, we will show that it admits a unique limit value. This is related to the uniqueness of the solution of (1.2).

\subsection{Existence and uniqueness of the solution of (1.2)}

Existence of the solutions of (1.2) is a consequence of Proposition 3.1, since the limit values of $\left(Z^{n}\right)_{n \in \mathbb{N}^{*}}$ are solutions. Proposition 3.2 deals with the uniqueness problem.

Proposition 3.2. Let $\xi_{0} \in \mathcal{M}_{F}\left(\mathbb{R}_{+}^{d}\right)$ be a deterministic initial condition. Let $\left(\xi_{t}^{1}\right)_{t \in \mathbb{R}_{+}},\left(\xi_{t}^{2}\right)_{t \in \mathbb{R}_{+}}$be two solutions of (1.2) in $\mathcal{C}\left(\mathbb{R}_{+}, \mathcal{M}_{F}\left(\mathbb{R}_{+}^{d}\right)\right)$ starting from $\xi_{0}$. Under Assumptions 1, 2, 4, we have: $\forall t \in \mathbb{R}_{+}, \xi_{t}^{1}(d a)=\xi_{t}^{2}(d a)$. 
Proof. The solutions of (1.2) are continuous with bounded masses on finite time intervals: $\forall t \geq 0,\left\langle\xi_{t}, 1\right\rangle \leq$ $\left\langle\xi_{0}, 1\right\rangle+\int_{0}^{t} \bar{b}\left\langle\xi_{s}, 1\right\rangle \mathrm{d} s \leq\left\langle\xi_{0}, 1\right\rangle \exp (\bar{b} t)<+\infty$. For $T>0$, we can define $A_{T}:=\sup _{t \in[0, T]}\left\langle\xi_{t}^{1}+\xi_{t}^{2}, 1\right\rangle<+\infty$. Let $\phi \in \mathcal{C}_{b}^{1}\left(\mathbb{R}_{+}^{d}, \mathbb{R}\right)$ such that $\|\phi\|_{\infty} \leq 1$. For $t \in[0, T]$, we can define $\forall a \in \mathbb{R}_{+}^{d}, \forall s \in \mathbb{R}_{+}, f(a, s)=\phi(A(t, s, a))$. By Proposition 2.2: $\forall i \in\{1,2\}, \forall t \in[0, T]$,

$$
\left\langle\xi_{t}^{i}, \phi\right\rangle=\int_{\mathbb{R}_{+}^{d}} \phi(A(t, 0, a)) \xi_{0}^{i}(d a)+\int_{0}^{t} \int_{\mathbb{R}_{+}^{d}}\left[\phi(A(t, s, 0)) b(a)-\phi(A(t, s, a)) d\left(a, \xi_{s}^{i} U(a)\right)\right] \xi_{s}^{i}(d a) \mathrm{d} s .
$$

Hence: $\forall t \in[0, T]$,

$$
\begin{aligned}
\left|\left\langle\xi_{t}^{1}-\xi_{t}^{2}, \phi\right\rangle\right| & \leq \int_{0}^{t}\left[\left|\int_{\mathbb{R}_{+}^{d}}\left(\phi(A(t, s, 0)) b(a)-\phi(A(t, s, a)) d\left(a, \xi_{s}^{1} U(a)\right)\right)\left(\xi_{s}^{1}(d a)-\xi_{s}^{2}(d a)\right)\right|\right. \\
& \left.+\left|\int_{\mathbb{R}_{+}^{d}} \phi(A(t, s, a))\left(d\left(a, \xi_{s}^{1} U(a)\right)-d\left(a, \xi_{s}^{2} U(a)\right)\right) \xi_{s}^{2}(d a)\right|\right] \mathrm{d} s \leq C(T) \int_{0}^{t}\left\|\xi_{s}^{1}-\xi_{s}^{2}\right\|_{T V} \mathrm{~d} s,
\end{aligned}
$$

where $C(T)=\bar{b}+\bar{d}\left(1+A_{T}\right)+A_{T} L_{d} \bar{U}$. By taking the sup in $\phi$ and by noticing that every function $\phi \in \mathcal{C}_{b}\left(\mathbb{R}_{+}^{d}, \mathbb{R}\right)$ is the limit for the bounded pointwise convergence of a sequence of functions of $\mathcal{C}_{b}^{1}\left(\mathbb{R}_{+}^{d}, \mathbb{R}\right)$ :

$$
\forall t \in[0, T],\left\|\xi_{t}^{1}-\xi_{t}^{2}\right\|_{T V} \leq C(T) \int_{0}^{t}\left\|\xi_{s}^{1}-\xi_{s}^{2}\right\|_{T V} \mathrm{~d} s .
$$

The result is given by Gronwall lemma, and by the fact that $T$ is arbitrary.

Corollary 3.3. Under Assumptions 1, 2, 4 and for every initial condition $\xi_{0} \in \mathcal{M}_{F}\left(\mathbb{R}_{+}^{d}\right)$, equation (1.2) admits a unique solution $\left(\xi_{t}\right)_{t \in \mathbb{R}_{+}} \in \mathcal{C}\left(\mathbb{R}_{+}, \mathcal{M}_{F}\left(\mathbb{R}_{+}^{d}\right)\right)$ to which the sequence $\left(Z^{n}\right)_{n \in \mathbb{N}^{*}}$ of $\mathbb{D}$ converges in probability.

\subsection{Absolute continuity of the solutions of (1.2)}

Let us now study the existence of densities for the measures $\xi_{t}(d a)$ with respect to the Lebesgue measure of $\mathbb{R}_{+}^{d}$. If they exist, they define a weak solution of (1.5) which generalizes the McKendrick and Von Foerster equations that are classical in demography. In the case of a scalar age we have the propagation of the absolute continuity for positive times (Prop. 3.4). However, when many ages are taken into account, densities do not exist any more (Rem. 3.5).

Proposition 3.4. In the case $d=1$ : under Assumptions 1, 2, 4, if $\xi_{0}(d a)=m_{0}(a) d a$ is absolutely continuous with respect to the Lebesgue measure on $\mathbb{R}_{+}$, then, for every $t \in \mathbb{R}_{+}, \xi_{t}(d a)$ also admits a density $m_{t}(a)$.

Proof. Let $t \in \mathbb{R}_{+}$and let $\phi \in \mathcal{C}_{b}^{1}\left(\mathbb{R}_{+}, \mathbb{R}_{+}\right)$non-negative. For $f$ defined by $\forall s \in \mathbb{R}_{+}, \forall a \in \mathbb{R}_{+}, f(a, s)=$ $\phi(A(t, s, a))$, where $A$ is defined in (2.2), we have:

$$
\left\langle\xi_{t}, \phi\right\rangle \leq \int_{\mathbb{R}_{+}} \phi(A(t, 0, a)) \xi_{0}(d a)+\int_{0}^{t} \int_{\mathbb{R}_{+}} \phi(A(t, s, 0)) b(a) \xi_{s}(d a) \mathrm{d} s
$$

since $\phi$ is non-negative. Let us consider the first term. By Point (ii) of Proposition 2.1, the map $a \mapsto A(t, 0, a)$ defines a $\mathcal{C}^{1}$-diffeomorphism from $\mathbb{R}_{+}$into $\left[A(t, 0,0),+\infty\left[\right.\right.$. Let us denote by $A^{-1}(t, \alpha)$ the inverse diffeomorphism and by $J A^{-1}(t, \alpha)$ its Jacobian matrix. Using the change of variable associated with $a \mapsto A(t, 0, a)$ :

$$
\int_{\mathbb{R}_{+}} \phi(A(t, 0, a)) m_{0}(a) \mathrm{d} a=\int_{A(t, 0,0)}^{+\infty} \phi(\alpha) m_{0}\left(A^{-1}(t, \alpha)\right)\left|J A^{-1}(t, \alpha)\right| \mathrm{d} \alpha .
$$


For the second term, we consider the change of variable given by the $\mathcal{C}^{1}$-diffeomorphism $s \mapsto A(t, s, 0)$. We denote by $\widetilde{A^{-1}}(t, \alpha)$ its inverse diffeomorphism and by $\widetilde{A^{-1}}(t, \alpha)$ its Jacobian matrix:

$$
\int_{0}^{t} \int_{\mathbb{R}_{+}} \phi(A(t, s, 0)) b(a) \xi_{s}(d a) \mathrm{d} s=\int_{0}^{A(t, 0,0)} \int_{\mathbb{R}_{+}} \phi(\alpha) b(a)\left|J \widetilde{A^{-1}}(t, \alpha)\right| \xi_{\widetilde{A^{-1}}(t, \alpha)}(d a) \mathrm{d} \alpha .
$$

From (3.3) and (3.4), we deduce that:

$$
\begin{gathered}
0 \leq \int_{\mathbb{R}_{+}} \phi(a) \xi_{t}(d a) \leq \int_{0}^{+\infty} \phi(\alpha) H(\alpha, t) \mathrm{d} \alpha, \text { where: } \\
H(\alpha, t)=\mathbf{1}_{A(t, 0,0) \leq \alpha} m_{0}\left(A^{-1}(t, \alpha)\right)\left|J A^{-1}(t, \alpha)\right|+\mathbf{1}_{\alpha \leq A(t, 0,0)} \int_{\mathbb{R}_{+}} b(a)\left|J \widetilde{A^{-1}}(t, \alpha)\right| \xi_{\widetilde{A^{-1}}(t, \alpha)}(d a) \mathrm{d} \alpha .
\end{gathered}
$$

The function $H$ is non-negative and integrable, since the right hand side of (3.5) equals the right hand side of (3.2), which is finite. Since every $\left(\xi_{t}(d a)+H(a, t) d a\right)$-a.e. non-negative bounded function can be approximated in $L^{1}\left(\mathbb{R}_{+}, \xi_{t}(d a)+H(a, t) d a\right)$ by a sequence of functions of $\mathcal{C}_{b}^{1}\left(\mathbb{R}_{+}, \mathbb{R}_{+}\right)$(see Rudin [44], p. 69, $\xi_{t}(d a)+H(a, t) d a$ being a finite measure), we deduce from (3.5) that the measure $\xi_{t}(d a)$, dominated by a measure that is absolutely continuous with respect to the Lebesgue measure, is itself absolutely continuous.

Remark 3.5. In the case where $d>1$, the map $t \in \mathbb{R}_{+} \mapsto A(t, 0,0) \in \mathbb{R}_{+}^{d}$ does not define a bijection any more, and the ages of individuals born after $s=0$ at time $t$ belong to the set $\left\{A(t, s, 0) \in \mathbb{R}_{+}^{d}, s \in[0, t]\right\}$, which is a one-dimensional variety of $\mathbb{R}_{+}^{d}$. Thus, the measures $\xi_{t}$ can not be absolutely continuous with respect to the Lebesgue measure on $\mathbb{R}_{+}^{d}$.

The following Proposition linking $(1.2)$ and $(1.3,1.4)$ is proved in Section 3.2.4 of [47]:

Proposition 3.6. For $d=1$ and under the Assumptions of Proposition 3.4, the weak function solution $(a, t) \mapsto$ $m(a, t)$ of (1.2) is well defined. If this solution belongs to $\mathcal{C}_{b}^{1}\left(\mathbb{R}_{+}^{2}, \mathbb{R}_{+}\right)$and if $\forall t \in \mathbb{R}_{+}, \frac{\partial}{\partial t} \int_{\mathbb{R}_{+}} m(a, t) \mathrm{d} a=$ $\int_{\mathbb{R}_{+}} \frac{\partial m}{\partial t}(a, t) \mathrm{d} a$, then it is the classical solution of (1.3, 1.4).

Proof. We replace $\xi_{s}(d a)$ in (1.2) by $m(a, s) d a$ and differentiate with respect to time. $\forall t \in \mathbb{R}_{+}$,

$$
\frac{\partial}{\partial t} \int_{\mathbb{R}_{+}} f(a) m(a, t) \mathrm{d} a=\int_{\mathbb{R}_{+}}\left[v(a) \frac{\partial f}{\partial a}(a)+f(0) b(a)-f(a) d\left(a, \int_{\mathbb{R}_{+}} U(a, \alpha) m(\alpha, t) \mathrm{d} \alpha\right)\right] m(a, t) \mathrm{d} a .
$$

Let us first consider test functions $f \in \mathcal{C}_{b, K}^{1}\left(\mathbb{R}_{+}^{*}, \mathbb{R}\right)$ bounded with compact support in $\mathbb{R}_{+}^{*}$. In particular $f(0)=0$. By Fubini theorem and by an integration by parts formula for the aging term: $\forall t \in \mathbb{R}_{+}$,

$$
\int_{\mathbb{R}_{+}} \frac{\partial f}{\partial a}(a) v(a) m(a, t) \mathrm{d} a=[f(a) v(a) m(a, t)]_{a=0}^{+\infty}-\int_{\mathbb{R}_{+}} f(a) \frac{\partial}{\partial a}(v(a) m(a, t)) \mathrm{d} a .
$$

Since for every $t \in \mathbb{R}_{+}$, the measure $\xi_{t}(d a)=m(a, t) d a$ is a finite measure, $\lim _{a \rightarrow+\infty} m(a, t)=0$ and the bracket in the right hand side equals to zero. From (3.6) and (3.7), we obtain that $d a$-almost surely on $\mathbb{R}_{+}^{*}$, and $\forall t \in \mathbb{R}_{+}$,

$$
\frac{\partial}{\partial t} m(a, t)+\frac{\partial}{\partial a}(v(a) m(a, t))=-d\left(a, \int_{\mathbb{R}_{+}} U(a, \alpha) m(\alpha, t) \mathrm{d} \alpha\right) m(a, t) .
$$


Let us now consider test functions $f \in \mathcal{C}_{b}^{1}\left(\mathbb{R}_{+}, \mathbb{R}\right)$ with arbitrary support. From (3.7) and (3.6): $\forall t \in \mathbb{R}_{+}$,

$$
\begin{aligned}
\int_{\mathbb{R}_{+}}\left[\frac{\partial}{\partial t} m(a, t)+\frac{\partial}{\partial a}(v(a) m(a, t))\right] f(a) \mathrm{d} a & =-f(0) v(0) m(0, t) \\
+ & \int_{\mathbb{R}_{+}}\left[f(0) b(a)-f(a) d\left(a, \int_{\mathbb{R}_{+}} U(a, \alpha) m(\alpha, t) \mathrm{d} \alpha\right)\right] m(a, t) \mathrm{d} a
\end{aligned}
$$

We simplify (3.9) with (3.8) and: $\forall t \in \mathbb{R}_{+}, f(0) v(0) m(0, t)=\int_{\mathbb{R}_{+}} f(0) b(a) m(a, t) \mathrm{d} a$. This gives the boundary condition in (1.4).

\section{EXPONENTIAL DEVIATIONS}

In this section, we set $T>0$ and study the exponential deviations associated with the convergence of $\left(Z^{n}\right)_{n \in \mathbb{N}^{*}}$ to the solution $\xi$ of (1.2) in $\mathbb{D}_{T}$ (Cor. 3.3). More precisely, we look for estimates of $(1 / n) \log \mathbb{P}\left(Z^{n} \in B\right)$, for any Borel set $B \subset \mathbb{D}_{T}$, when $n \rightarrow+\infty$. Applications will be considered in Section 5 .

A first difficulty arises from the fact that $\mathcal{M}_{F}\left(\mathbb{R}_{+}^{d}\right)$ is not compact. The mass of the microscopic process is not necessarily bounded. The exponential tightness of $\left(Z_{. \wedge \zeta_{N}^{n}}^{n}\right)_{n \in \mathbb{N}^{*}}$ on $\mathbb{D}_{T}$ is classical and can be established by adapting techniques from Dawson and Gärtner [11] and Graham and Méléard [21]. The relaxation of the localization by $\zeta_{N}^{n}$ relies on exponential deviation inequalities proved in Lemmas 4.6 and 4.7.

The lower bound issue is treated in Section 4.3. A second difficulty comes from the fact that the events describing the dynamics are of different natures (births, deaths) and that they are associated with nonlinear rates. Following the approach of Léonard [32], Kipnis and Léonard [31], we base our proof on the use of a Riesz theorem in Orlicz spaces, which gives us a representation of the trajectories in the domain of the action functional as solutions of perturbations of PDE (1.2) and on a Girsanov theorem. A key point in the proof is the use of a density theorem due to Ekeland Temam [14], Bishop Phelps [5] and Israel [24].

Notation. We define $E:=\mathbb{R}_{+}^{d} \times\{0,1\}$. With each function $f \in \mathcal{B}\left(\mathbb{R}_{+}^{d} \times[0, T], \mathbb{R}\right)$, we can associate a map $\psi(f)$ defined on $E \times[0, T]$ by: $\forall(a, u, t) \in E \times[0, T]$,

$$
\psi(f)(a, u, t)=f(0, t) \mathbf{1}_{u=0}-f(a, t) \mathbf{1}_{u=1} .
$$

For $t \in[0, T]$ and $z \in \mathbb{D}_{T}$, let us introduce the following positive finite measure on $E$ :

$$
m_{t}^{z, T}(d a, d u)=\left[b(a) \delta_{0}(d u)+d\left(a, z_{t-} U(a)\right) \delta_{1}(d u)\right] z_{t_{-}}(d a) .
$$

The Laplace transform $\rho$ of the standard centered Poisson law and its Legendre transform are:

$$
\rho(x)=e^{x}-x-1, \quad \rho^{*}(y)=\left\{\begin{array}{cc}
(y+1) \log (y+1)-y & \text { if } y>-1 \\
1 & \text { if } y=-1 \\
+\infty & \text { if } y<-1
\end{array}\right.
$$

$\rho^{*}$ is non-negative and vanishes only at $y=0$. The functions $\rho$ and $\rho^{*}$ are convex conjugated. For $\alpha \in\left\{\rho, \rho^{*}\right\}$ and $z \in \mathbb{D}_{T}$, we define the following Orlicz norm on the space of real Borel functions on $E \times \mathbb{R}_{+}$:

$$
\|g\|_{\alpha, z}=\inf \left\{\kappa>0, \int_{0}^{T} \int_{E} \alpha\left(\frac{|g(a, u, s)|}{\kappa}\right) m_{s}^{z, T}(d a, d u) \mathrm{d} s \leq 1\right\} .
$$

The set of functions with finite Orlicz norms are denoted by $L^{\rho, z}$ and $L^{\rho^{*}, z}$. The closure $E^{\rho, z}\left(E \times \mathbb{R}_{+}\right)$of the space of Borel bounded functions for the norm $\|\cdot\|_{\rho, z}$ is strictly included in $L^{\rho, z}$ (see [31] Sect. 3, for instance) 
and admits a topological dual which can be identified with $L^{\rho^{*}, z}$ thanks to the following representation theorem:

Theorem 4.1 (Riesz theorem in Orlicz spaces). (See Rao et Ren [42], p. 93 and following or Kipnis and Léonard [31]) For every continuous linear form $\ell$ on $E^{\rho, z}\left(E \times \mathbb{R}_{+}\right)$, there exists a function $h \in L^{\rho^{*}, z}$ such that:

$$
\forall g \in E^{\rho, z}\left(E \times \mathbb{R}_{+}\right), \ell(g)=\int_{0}^{T} \int_{E} g(a, u, s) h(a, u, s) m_{s}^{z, T}(d a, d u) \mathrm{d} s .
$$

\subsection{Main result}

The main result of this section is enounced in Theorem 4.3.

Assumption 5. We assume that Assumptions 3 are satisfied and that:

1. The sequence $\left(Z_{0}^{n}\right)_{n \in \mathbb{N}^{*}}$ is deterministic and converges in $\left(\mathcal{M}_{F}\left(\mathbb{R}_{+}^{d}\right), w\right)$ to a measure $\xi_{0}$.

2. $\exists C_{0}>0,\left\langle\xi_{0}, 1+|a|\right\rangle<C_{0}$ and $\exists n_{0} \in \mathbb{N}^{*}, \forall n \geq n_{0},\left\langle Z_{0}^{n}, 1+|a|\right\rangle<C_{0}$.

The Assumption of Point 1 is made for simplification. It can be weakened by assuming that $\left(Z_{0}^{n}\right)_{n \in \mathbb{N}^{*}}$ converges in law in $\left(\mathcal{M}_{F}\left(\mathbb{R}_{+}^{d}\right), w\right)$ to $\xi_{0}$.

We now define the action functional that appears in our deviation result.

Definition 4.2. For $\xi_{0} \in \mathcal{M}_{F}\left(\mathbb{R}_{+}^{d}\right)$ and $z \in \mathbb{D}_{T}$, let us define $\mathcal{I}_{\xi_{0}}^{T}(z)$ by:

$$
\mathcal{I}_{\xi_{0}}^{T}(z)=\left\{\begin{array}{c}
\sup _{f \in \mathcal{C}_{b}^{1}\left(\mathbb{R}_{+}^{d} \times[0, T], \mathbb{R}\right)} \mathcal{I}^{f, T}(z), \text { if } z_{0}=\xi_{0}, \quad \text { with: } \mathcal{I}^{f, T}(z)=\ell^{T}(f, z)-c^{T}(f, z), \\
+\infty, \text { else, }
\end{array}\right.
$$

where $\ell^{T}(f, z)$ and $c^{T}(f, z)$ are defined for $\left(f:(a, s) \mapsto f_{s}(a)\right) \in \mathcal{C}_{b}^{1}\left(\mathbb{R}_{+}^{d} \times[0, T], \mathbb{R}\right)$ by:

$$
\begin{aligned}
& \ell^{T}(f, z)=\left\langle z_{T}, f_{T}\right\rangle-\left\langle z_{0}, f_{0}\right\rangle-\int_{0}^{T}\left[\int_{\mathbb{R}_{+}^{d}}\left(v(a) \nabla_{a} f_{s}(a)+\frac{\partial f_{s}}{\partial s}(a)\right) z_{s}(d a)-\int_{E} \psi(f) \mathrm{d} m_{s}^{z, T}\right] \mathrm{d} s, \\
& c^{T}(f, z)=\int_{0}^{T} \int_{E} \rho(\psi(f)(a, u, s)) m_{s}^{z, T}(d a, d u) \mathrm{d} s .
\end{aligned}
$$

Theorem 4.3. Under Assumptions 1, 2, 5,

(i) Let $z \in \mathbb{D}_{T}$ such that $\mathcal{I}_{\xi_{0}}^{T}(z)<+\infty$. There exists $h^{z} \in L^{\rho^{*}, z}$ such that $z$ is the solution of: $\forall(f:(a, s) \mapsto$ $\left.f_{s}(a)\right) \in \mathcal{C}_{b}^{1}\left(\mathbb{R}_{+}^{d} \times \mathbb{R}_{+}, \mathbb{R}\right), \forall t \in[0, T]$,

$$
\left\langle z_{t}, f_{t}\right\rangle=\left\langle z_{0}, f_{0}\right\rangle+\int_{0}^{t}\left\langle z_{s}, v \nabla_{a} f_{s}+\frac{\partial f_{s}}{\partial s}\right\rangle \mathrm{d} s+\int_{0}^{t} \int_{E}\left(1+h^{z}\right) \psi(f) \mathrm{d} m_{s}^{z, T} \mathrm{~d} s .
$$

We then have the following non-variational representation of the action functional:

$$
\mathcal{I}_{\xi_{0}}^{T}(z)=\int_{0}^{T} \int_{E} \rho^{*}\left(h^{z}(a, u, s)\right) m_{s}^{z, T}(d a, d u) \mathrm{d} s .
$$

(ii) Let:

$$
\mathfrak{G}=\left\{z \in \mathbb{D}_{T} \mid \mathcal{I}_{\xi_{0}}^{T}(z)<+\infty, \quad \text { and } \quad h^{z} \in L^{\infty}(E \times[0, T], \mathbb{R})\right\} .
$$

The sequence $\left(Z^{n}\right)_{n \in \mathbb{N}^{*}}$ of $\mathbb{D}_{T}$ satisfies the following deviation inequalities: $\forall B$ Borel set of $\mathbb{D}_{T}$,

$$
\text { - } \inf _{z \in \bar{B} \cap \mathfrak{G}} \mathcal{I}_{\xi_{0}}^{T}(z) \leq \liminf _{n \rightarrow+\infty} \frac{1}{n} \log \mathbb{P}\left(Z^{n} \in B\right) \leq \limsup _{n \rightarrow+\infty} \frac{1}{n} \log \mathbb{P}\left(Z^{n} \in B\right) \leq-\inf _{z \in \bar{B}} \mathcal{I}_{\xi_{0}}^{T}(z),
$$

with the convention $\inf \emptyset=+\infty$. 
Remark 4.4. (4.7) corresponds to a perturbation of the birth and death rates of (1.2) by factors $\left(1+h^{z}(a, 0, s)\right)$ and $\left(1+h^{z}(a, 1, s)\right)$ which are different (and hence the introduction of the mark $\left.u \in\{0,1\}\right)$. We will prove in Proposition 4.12 (Point 4) that these factors are nonnegative.

The upper bound of (4.10) is a large deviation upper bound. The lower bound is local, the infimum being taken on $\stackrel{\leftrightarrow}{B} \cap \mathfrak{G}$ and not $\stackrel{\leftrightarrow}{B}$. In the proof of the lower bound, we will be lead to regularize $h^{z}$ by approximating it with a sequence $\left(h_{m}\right)_{m \in \mathbb{N}^{*}}$ of perturbations with particular forms, differentiable and bounded. With each $h_{m}$, we can associate the solution $z^{m}$ of the evolution equation (4.7) perturbed by $h_{m}$. The convergence of $z^{m}$ to $z$ when $h^{z}$ is bounded is proved in Proposition 4.16, the general case is still open. In the works which have inspired us [31,32] the similar difficulty is encountered, but under a different form: the problem is a uniqueness problem for the perturbed equation (4.7) and not a convergence problem.

The local minoration that we obtain is still a useful result, that we will use in Section 5. It provides an information as soon as $\stackrel{B}{B} \cap \mathfrak{G} \neq \emptyset$, in which case, the lower bound of (4.10) is not $-\infty$. This is the case when $\stackrel{\circ}{B}$ contains the solution of (1.2) which corresponds to a zero perturbation for instance.

\subsection{Exponential tightness and large deviation upper bound}

We prove the exponential tightness of the laws of $\left(Z^{n}\right)_{n \in \mathbb{N}^{*}}$ on $\mathbb{D}_{T}$. The large deviation upper bound is then a generalization of Theorem 4.4.2 and Lemma 4.4.5 of Dembo and Zeitouni [12], obtained by replacing the limits with limsup.

Recall that the continuity modulus for $y \in \mathbb{D}([0, T], \mathbb{R})$ and $\delta \in] 0,1[$ is defined by:

$$
w^{\prime}(y, \delta)=\inf _{\left\{t_{l}\right\}}\left(\operatorname { m a x } _ { 1 < i \leq \operatorname { c a r d } \{ t _ { l } \} } w \left(y , [ t _ { i - 1 } , t _ { i } [ ) ) \quad \text { where } w \left(y,\left[t_{i-1}, t_{i}[)=\sup _{s, t \in\left[t_{i-1}, t_{i}[\right.}\left|y_{s}-y_{t}\right|,\right.\right.\right.\right.
$$

and where the infimum is considered on the subdivisions $0=t_{0}<t_{1} \cdots<t_{\text {card }\left\{\mathrm{t}_{1}\right\}}=T$ such that $\forall i \in$ $\llbracket 1, \operatorname{card}\left\{t_{l}\right\} \rrbracket, t_{i}-t_{i-1}>\delta$.

Proposition 4.5. Under Assumptions 1, 2, 5:

(i) Let $\left(\varphi_{r}\right)_{r \in \mathbb{N}^{*}}$ be a countable dense family of $\mathcal{C}_{0}^{1}\left(\mathbb{R}_{+}^{d}, \mathbb{R}\right)$, and let us set $\varphi_{0} \equiv 1$. $\forall L>0, \forall N>0, \exists \mathcal{K}_{L, N}^{1}$ closed subset of $\mathbb{D}_{T}, \exists n_{0} \in \mathbb{N}^{*}, \forall n \geq n_{0}$ :

$$
\mathbb{P}\left(Z_{. \wedge \zeta_{N}^{n}}^{n} \notin \mathcal{K}_{L, N}^{1}\right) \leq \frac{\mathrm{e}^{-n L}\left(2-\mathrm{e}^{-L}\right)}{\left(1-\mathrm{e}^{-L}\right)^{2}} \text { and such that: } \forall r \in \mathbb{N}, \lim _{\delta \rightarrow 0} \sup _{z \in \mathcal{K}_{L, N}^{1}} w^{\prime}\left(\left\langle z, \varphi_{r}\right\rangle, \delta\right)=0 .
$$

(ii) $\forall L>0, \exists \mathcal{K}_{L}^{2}$ compact subset of $\left(\mathcal{M}_{F}\left(\mathbb{R}_{+}^{d}\right), w\right)$,

$$
\limsup _{n \rightarrow+\infty} \frac{1}{n} \log \left(\mathbb{P}\left(\exists t \in[0, T], Z_{t}^{n} \notin \mathcal{K}_{L}^{2}\right)\right) \leq-L .
$$

(iii) As a consequence of (i) and (ii), the sequence of laws of $\left(Z^{n}\right)_{n \in \mathbb{N}^{*}}$ is exponentially tight on $\mathbb{D}_{T}: \forall L>$ $0, \exists K_{L} \subset \mathbb{D}_{T}$ compact, $\lim \sup _{n \rightarrow+\infty} \frac{1}{n} \log \mathbb{P}\left(Z^{n} \notin K_{L}\right) \leq-L$.

The measure-valued process $Z_{. \wedge \zeta_{N}^{n}}^{n}$ has marginals of masses bounded by $N>0$. The proof of Point (i) is an adaption of the proof in [11] (see [47] for a detailed proof). It relies on the proof of the following estimate, obtained by an adaptation of techniques used in [20,21]: $\forall \varepsilon>0, \forall N>0, \forall c>0, \forall \delta>0, \forall n \in \mathbb{N}, \forall \varphi \in \mathcal{C}_{b}^{1}\left(\mathbb{R}_{+}^{d}\right)$,

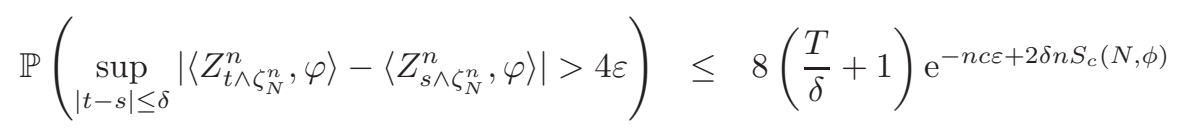

where $S_{c}(N, \varphi)=\left[\rho\left(c\|\varphi\|_{\infty}\right) N(\bar{b}+\bar{d}(1+N))\right] \vee\left[c\left(\bar{v} 2 N\left\|\nabla_{a} \varphi\right\|_{\infty}+N\|\varphi\|_{\infty}(\bar{b}+\bar{d}(1+N))\right)\right]$. The upper bound in (4.13) depends on $N$, and we can not take the limit in $N \rightarrow+\infty$. 
The end of Proposition 4.5 makes the object of the end of this section. We generalize an argument used by Dawson Gärtner [11] in the case of continuous probability-valued processes. We prove that there exists $N>0$ such that the probability $\mathbb{P}\left(\zeta_{N}^{n} \leq T\right)$ is exponentially small. Then, the exponential tightness of the laws of $\left(\left(Z_{t \wedge \zeta_{N}^{n}}^{n}\right)_{t \in[0, T]}\right)_{n \in \mathbb{N}^{*}}$ implies the exponential tightness of the laws of $\left(\left(Z_{t}^{n}\right)_{t \in[0, T]}\right)_{n \in \mathbb{N}^{*}}$.

We set $\phi_{0} \equiv 1$ and introduce a denumberable family $\mathfrak{K}=\left(\phi_{r}\right)_{r \in \mathbb{N}}$ such that for every $r \in \mathbb{N}^{*}, \phi_{r}$ is constant outside a compact set of $\mathbb{R}_{+}^{d}$, polynomial on this compact set, and bounded up and below by positive constants, and such that for every $\phi \in \mathcal{C}_{0}\left(\mathbb{R}_{+}^{d}, \mathbb{R}_{+}\right)$there exists a sub-sequence $\left(\phi_{\psi(r)}\right)_{r \in \mathbb{N}}$ of $\left(\phi_{r}\right)_{r \in \mathbb{N}}$ that converges uniformly to $\phi$ (see [47] for the existence of this sequence).

In order to establish (4.12), we will need the following lemma which shows that quantities such as $\sup _{t \in[0, T]}\left\langle Z_{t}^{n}, \phi\right\rangle$ for useful choices of $\phi$ remain bounded up to a set of exponentially small probability:

Lemma 4.6. Under Assumptions 1, 2, 5,

(i) $\forall L>0, \exists N=N\left(T, L, C_{0}\right)>0, \exists n_{0} \in \mathbb{N}^{*}, \forall n>n_{0}, \mathbb{P}\left(\tau_{N}^{n}>T\right) \leq \mathrm{e}^{-n L}, \quad$ and $\mathbb{P}\left(\zeta_{N}^{n}>T\right) \leq \mathrm{e}^{-n L}$, $C_{0}$ being defined in Point 2 of Assumptions 3.

(ii) $\forall \phi \in \mathfrak{K}, \forall L>0, \exists C=C\left(\phi, T, L, C_{0}\right)>0, \exists n_{0} \in \mathbb{N}^{*}, \forall n>n_{0}, \mathbb{P}\left(\sup _{t \in[0, T]}\left\langle Z_{t}^{n}, \phi\right\rangle>C\right) \leq \mathrm{e}^{-n L}$.

The proof of Lemma 4.6 is based on the following Lemma 4.7, where we establish a sufficient condition (4.14) to obtain the announced upper bounds.

Lemma 4.7. Under Assumptions 1, 2, 5, let us consider $C_{0}$ and $n_{0}$ defined in Assumptions 5 and $\phi \in$ $\mathcal{C}_{b}^{1}\left(\mathbb{R}_{+}^{d}, \mathbb{R}\right)$ satisfying $\exists C(\phi)>0, \forall a \in \mathbb{R}_{+}^{d},|\phi(a)| \leq C(\phi)(1+|a|)$. If there exists $\gamma=\gamma\left(\phi, T, C_{0}\right)>0$ such that $\forall t \in[0, T], \forall n>n_{0}, \mathbb{P}$-a.s.:

$$
\int_{E} \psi(\phi) \mathrm{d} m_{t}^{Z^{n}, T}+\left\langle Z_{t}^{n}, v \nabla_{a} \phi\right\rangle-\gamma\left\langle Z_{t}^{n}, \phi\right\rangle \leq-\int_{E} \mathrm{e}^{\gamma t} \rho\left(\psi(\phi) \mathrm{e}^{-\gamma t}\right) \mathrm{d} m_{t}^{Z^{n}, T},
$$

then: $\forall L>0, \exists C=C\left(\phi, T, L, C_{0}\right)>0, \forall n>n_{0}$,

$$
\mathbb{P}\left(\sup _{t \in[0, T]}\left\langle Z_{t}^{n}, \phi\right\rangle>C\right) \leq \mathrm{e}^{-n L}
$$

Proof of Lemma 4.7. We are inspired by techniques from [11] for continuous probability-valued processes, and consider the semi-martingale $\left(\mathrm{e}^{-\gamma t}\left\langle Z_{t}^{n}, \phi\right\rangle\right)_{t \in[0, T]}$. By Point 2 of Assumptions $5,2 C_{0} C(\phi)+\left\langle\xi_{0}-Z_{0}^{n}, \phi\right\rangle \geq 0$ for sufficiently large $n$. Then:

$$
\begin{aligned}
& \mathbb{P}\left(\sup _{t \in[0, T]}\left\langle Z_{t}^{n}, \phi\right\rangle>\mathrm{e}^{\gamma T}\left(L+2 C_{0} C(\phi)+\left\langle\xi_{0}, \phi\right\rangle\right)\right) \\
= & \mathbb{P}\left(\exists t \in[0, T], \mathrm{e}^{-\gamma t}\left\langle Z_{t}^{n}, \phi\right\rangle>\mathrm{e}^{\gamma(T-t)}\left(L+2 C_{0} C(\phi)+\left\langle\xi_{0}, \phi\right\rangle\right)\right) \\
\leq & \mathbb{P}\left(\sup _{t \in[0, T]} \mathrm{e}^{-\gamma t}\left\langle Z_{t}^{n}, \phi\right\rangle-\left\langle Z_{0}^{n}, \phi\right\rangle>L+2 C_{0} C(\phi)+\left\langle\xi_{0}-Z_{0}^{n}, \phi\right\rangle\right) \text { since } \mathrm{e}^{\gamma(T-t)} \geq 1 \\
\leq & \mathbb{P}\left(\sup _{t \in[0, T]} M_{t}^{n, \gamma, \phi}+\int_{0}^{t} \mathrm{e}^{-\gamma s}\left\langle Z_{s}^{n}, v \nabla_{a} \phi-\gamma \phi\right\rangle \mathrm{d} s+\int_{0}^{t} \int_{E} \mathrm{e}^{-\gamma s} \psi(\phi) \mathrm{d} m_{s}^{Z^{n}, T} \mathrm{~d} s>L\right),
\end{aligned}
$$

where:

$$
M_{t}^{n, \gamma, \phi}:=\mathrm{e}^{-\gamma t}\left\langle Z_{t}^{n}, \phi\right\rangle-\left\langle Z_{0}^{n}, \phi\right\rangle-\int_{0}^{t}\left\langle Z_{s}^{n}, v \nabla_{a} \phi-\gamma \phi\right\rangle \mathrm{e}^{-\gamma s} \mathrm{~d} s-\int_{0}^{t} \int_{E} \psi(\phi) \mathrm{e}^{-\gamma s} \mathrm{~d} m_{s}^{Z^{n}, T} \mathrm{~d} s
$$


is a local martingale starting from 0 (Point (iii) of Prop. 2.7). By the Assumption (4.14):

$$
\begin{aligned}
\int_{0}^{t}\left\langle Z_{s}^{n}, v \nabla_{a} \phi-\gamma \phi\right\rangle \mathrm{e}^{-\gamma s} \mathrm{~d} s+\int_{0}^{t} \int_{E} \psi(\phi) \mathrm{e}^{-\gamma s} \mathrm{~d} m_{s}^{Z^{n}, T} \mathrm{~d} s & \leq-\int_{0}^{t} \mathrm{e}^{-\gamma s}\left[\int_{E} \mathrm{e}^{\gamma s} \rho\left(\psi(\phi) \mathrm{e}^{-\gamma s}\right) \mathrm{d} m_{s}^{Z^{n}, T}\right] \mathrm{d} s \\
& =-\int_{0}^{t} \int_{E} \rho\left(\psi(\phi) \mathrm{e}^{-\gamma s}\right) \mathrm{d} m_{s}^{Z^{n}, T} \mathrm{~d} s=:-\frac{1}{n} \Xi_{t}^{n, \gamma, n \phi} .
\end{aligned}
$$

Thus:

$$
\mathbb{P}\left(\sup _{t \in[0, T]}\left\langle Z_{t}^{n}, \phi\right\rangle>\mathrm{e}^{\gamma T}\left(L+2 C_{0} C(\phi)+\left\langle\xi_{0}, \phi\right\rangle\right)\right) \leq \mathbb{P}\left(\sup _{t \in[0, T]} M_{t}^{n, \gamma, n \phi}-\Xi_{t}^{n, \gamma, n \phi}>n L\right) \leq \mathrm{e}^{-n L},
$$

by the Doob inequality and Point (ii) of Proposition 2.9.

To prove that the assertions of Lemma 4.6 hold, we will prove that the condition (4.14) of Lemma 4.7 is satisfied for proper choices of functions $\phi$.

Proof of Lemma 4.6. We begin with establishing a sufficient condition (Inequality (4.18)) for (4.14) to be satisfied. We obtain the results announced in Lemma 4.6 thanks to Lemma 4.7, by proving that this sufficient condition is satisfied for proper choices of functions $\phi$.

Let $t \in[0, T]$ and $n \in \mathbb{N}^{*}$. In the case where $\left\langle Z_{t}^{n}, 1\right\rangle=0$, the two members of (4.14) are zero. Assume that $\left\langle Z_{t}^{n}, 1\right\rangle>0$. Let $\phi \in \mathcal{C}^{1}\left(\mathbb{R}_{+}^{d}, \mathbb{R}_{+}\right)$be a positive function and let $\gamma>0$.

$$
\begin{aligned}
\mathrm{e}^{\gamma t} \rho\left(\psi(\phi) \mathrm{e}^{-\gamma t}\right)+\psi(\phi) & =\mathrm{e}^{\gamma t}\left(\exp \left(\psi(\phi) \mathrm{e}^{-\gamma t}\right)-\psi(\phi) \mathrm{e}^{-\gamma t}-1\right)+\psi(\phi) \\
& =\sum_{k=1}^{+\infty}[\psi(\phi)]^{k} \frac{\mathrm{e}^{-(k-1) \gamma t}}{k !}=\psi(\phi) \sum_{k=0}^{+\infty}[\psi(\phi)]^{k} \frac{\mathrm{e}^{-k \gamma t}}{k !(k+1)} .
\end{aligned}
$$

Since for every $k \in \mathbb{N}, 1 / 2^{k} \leq 1 /(k+1) \leq 1$, we have:

$$
\frac{1}{k !}\left[\frac{\mathrm{e}^{-\gamma t}}{2} \psi(\phi)\right]^{k} \leq[\psi(\phi)]^{k} \frac{\mathrm{e}^{-k \gamma t}}{k !(k+1)} \leq \frac{1}{k !}\left[\mathrm{e}^{-\gamma t} \psi(\phi)\right]^{k},
$$

summing over $k$ gives:

$$
\psi(\phi) \exp \left(\frac{\mathrm{e}^{-\gamma t}}{2} \psi(\phi)\right) \leq \mathrm{e}^{\gamma t} \rho\left(\psi(\phi) \mathrm{e}^{-\gamma t}\right)+\psi(\phi) \leq \psi(\phi) \exp \left(\mathrm{e}^{-\gamma t} \psi(\phi)\right) .
$$

Since $\phi$ is non-negative:

$$
\int_{E}\left[\mathrm{e}^{\gamma t} \rho\left(\psi(\phi) \mathrm{e}^{-\gamma t}\right)+\psi(\phi)\right] \mathrm{d} m_{t}^{Z^{n}, T} \leq \int_{\mathbb{R}_{+}^{d}} \phi(0) \exp \left(\mathrm{e}^{-\gamma t} \phi(0)\right) b(a) Z_{t}^{n}(d a) .
$$

A sufficient condition for (4.14) to be satisfied is that there exists $\gamma>0$ such that:

$$
\left\langle Z_{t}^{n}, v \nabla_{a} \phi-\gamma \phi\right\rangle+\int_{\mathbb{R}_{+}^{d}} \phi(0) \exp \left(\mathrm{e}^{-\gamma t} \phi(0)\right) b(a) Z_{t}^{n}(d a) \leq 0 .
$$

We now prove Point (i) of Lemma 4.6. Since $\mathbb{P}\left(\tau_{N}^{n} \leq T\right)=\mathbb{P}\left(\sup _{t \in[0, T]}\left\langle Z_{t}^{n}, 1\right\rangle \geq N\right)$, it is sufficient, by Lemma 4.7, to prove that (4.14) is satisfied for $\phi \equiv 1$. This is fulfilled since (4.18) holds for $\phi \equiv 1$ and $\gamma>e \bar{b}$. 
We proceed in a similar way to establish the deviation inequality for $\zeta_{N}^{n}$. For $\phi$ defined by: $\forall a \in \mathbb{R}_{+}^{d}, \phi(a)=$ $1+|a|=1+\sqrt{\sum_{i=1}^{d} a_{i}^{2}},(4.18)$ becomes:

$$
\int_{\mathbb{R}_{+}^{d}}\left(\exp \left(\mathrm{e}^{-\gamma t}\right) b(a)+\sum_{i=1}^{d} \frac{2 v_{i}(a) a_{i}}{|a|}-\gamma-\gamma|a|\right) Z_{t}^{n}(d a) \leq 0 .
$$

By Assumptions 1 and since $\left(\sum_{i=1}^{d} a_{i}\right)^{2} \leq C(d) \sum_{i=1}^{d} a_{i}^{2}$, we have $\sum_{i=1}^{d} \frac{2 v_{i}(a) a_{i}}{|a|} \leq 2 \bar{v}(\sqrt{C(d)}+|a|)$. Then, (4.18) is satisfied for $\gamma>e \bar{b}+\sqrt{C(d)} 2 \bar{v}$. By Lemma 4.7:

$$
\begin{aligned}
\limsup _{n \rightarrow+\infty} \frac{1}{n} \log \mathbb{P}\left(\zeta_{N}^{n} \leq T\right) & =\limsup _{n \rightarrow+\infty} \frac{1}{n} \log \mathbb{P}\left(\sup _{t \in[0, T]}\left\langle Z_{t}^{n}, 1\right\rangle \geq N \text { or } \sup _{t \in[0, T]} \int_{\mathbb{R}_{+}^{d}}|a| Z_{t}^{n}(d a) \geq N\right) \\
& \leq \limsup _{n \rightarrow+\infty} \frac{1}{n} \log \mathbb{P}\left(\sup _{t \in[0, T]}\left\langle Z_{t}^{n}, 1+|a|\right\rangle \geq N\right) \leq-L .
\end{aligned}
$$

This concludes the proof of Point (i).

Let us now consider Point (ii). Let $\phi \in \mathfrak{K}$. The condition (4.18) is satisfied if: $\forall a \in \mathbb{R}_{+}^{d}$,

$$
v(a) \nabla_{a} \phi(a)-\gamma \phi(a)+\phi(0) \exp (\phi(0)) b(a) \leq 0,
$$

which is satisfied as soon as:

$$
\gamma>\sup _{a \in \mathbb{R}_{+}^{d}}\left[\frac{1}{\phi(a)}\left(v(a) \nabla_{a} \phi(a)+\phi(0) \exp (\phi(0)) b(a)\right)\right] .
$$

By the definition of $\mathfrak{K}$, since the function $\phi$ is polynomial on a compact set and constant outside this set, it is possible to choose $\gamma$ satisfying (4.19) as the right member is bounded by a constant independent from $a \in \mathbb{R}_{+}^{d}$ :

$$
\lim _{|a| \rightarrow+\infty} \frac{\sum_{i=1}^{d}\left(1+a_{i}\right) \frac{\partial \phi}{\partial a_{i}}(a)}{\phi(a)} \leq C<+\infty
$$

Lemma 4.7 holds and concludes the proof.

We can now conclude the proof of the exponential tightness of the laws of $\left(Z^{n}\right)_{n \in \mathbb{N}^{*}}$.

Proof of Points (ii) and (iii) of Proposition 4.5. We begin with the proof of (ii). Let us introduce the following sets for $C>0$ and $\phi \in \mathcal{C}_{b}\left(\mathbb{R}_{+}^{d}, \mathbb{R}_{+}\right)$:

$$
\mathcal{K}_{\phi}(C)=\left\{z \in \mathcal{M}_{F}\left(\mathbb{R}_{+}^{d}\right) \mid\langle z, \phi\rangle \leq C\right\} .
$$

Let us prove that the set $\mathcal{K}_{L}^{2}:=\operatorname{adh}\left[\bigcap_{r \in \mathbb{N}} \mathcal{K}_{\phi_{r}}\left(C_{r}\right)\right]$, with $C_{r}:=C\left(\phi_{r}, T, r L \vee L, C_{0}\right)$ of Lemma 4.6 (ii), is compact in $\left(\mathcal{M}_{F}\left(\mathbb{R}_{+}^{d}\right), w\right)$ and that $(4.12)$ is satisfied.

Let $\left(z_{m}\right)_{m \in \mathbb{N}^{*}}$ be a sequence of $\bigcap_{r \in \mathbb{N}} \mathcal{K}_{\phi_{r}}\left(C_{r}\right)$. For every given $r \in \mathbb{N},\left(\left\langle z_{m}, \phi_{r}\right\rangle\right)_{m \in \mathbb{N}^{*}}$ is a non-negative real sequence upper bounded by $C_{r}$ and hence relatively compact.

By a diagonal procedure, we can extract from $\left(z_{m}\right)_{m \in \mathbb{N}^{*}}$ a subsequence, again denoted by $\left(z_{m}\right)_{m \in \mathbb{N}^{*}}$, such that for every $r \in \mathbb{N},\left(\left\langle z_{m}, \phi_{r}\right\rangle\right)_{m \in \mathbb{N}^{*}}$ converges to a limit $\ell\left(\phi_{r}\right)$. Let $g \in \mathcal{C}_{0}\left(\mathbb{R}_{+}^{d}, \mathbb{R}_{+}\right)$. By choice of $\mathfrak{K}$, there exists a subsequence $\left(\phi_{\psi(r)}\right)_{r \in \mathbb{N}}$ of $\left(\phi_{r}\right)_{r \in \mathbb{N}}$ that converges uniformly to $g$. The sequence $\left(\ell\left(\phi_{\psi(r)}\right)\right)_{r \in \mathbb{N}}$ is then a Cauchy sequence in $\mathbb{R}$ and we can define the limit $\ell(g)=\lim _{r \rightarrow+\infty} \ell\left(\phi_{\psi(r)}\right)$, which does not depend on the choice of 
the subsequence $\left(\phi_{\psi(r)}\right)_{r \in \mathbb{N}}$. With a non-positive function $g \in \mathcal{C}_{0}\left(\mathbb{R}_{+}^{d}, \mathbb{R}_{-}\right)$, we associate $\ell(g):=-\ell(-g)$. With $g \in \mathcal{C}_{0}\left(\mathbb{R}_{+}^{d}, \mathbb{R}\right)$, we associate $\ell(g)=\ell\left([g]_{+}\right)-\ell\left([g]_{-}\right)$, where $[\cdot]_{+}$and $[\cdot]_{-}$denote the positive and negative parts.

If $\ell(1) \neq 0$, we can prove that the map $g \in \mathcal{C}_{0}\left(\mathbb{R}_{+}^{d}, \mathbb{R}\right) \mapsto \ell(g) / \ell(1) \in \mathbb{R}$ is well-defined, linear, continuous, of norm 1 and non-negative. Thanks to the Riesz representation Theorem (see Rudin [44], Th. 6.19 p. 130), there exists a unique probability measure $\nu$, such that $\forall g \in \mathcal{C}_{0}\left(\mathbb{R}_{+}^{d}, \mathbb{R}\right), \ell(g)=\ell(1) \int_{\mathbb{R}_{+}^{d}} g(a) \nu(d a)$. By construction, for every $g \in \mathcal{C}_{0}\left(\mathbb{R}_{+}^{d}, \mathbb{R}\right),\left(\left\langle z_{m}, g\right\rangle\right)_{m \in \mathbb{N}^{*}}$ converges to $\langle\ell(1) \nu, g\rangle$. Since $\left(\left\langle z_{m}, 1\right\rangle\right)_{m \in \mathbb{N}^{*}}$ converges to $\ell(1)=\langle\ell(1) \nu, 1\rangle$, $\left(z_{m}\right)_{m \in \mathbb{N}^{*}}$ converges weakly to $\ell(1) \nu$ (see [36]).

If $\ell(1)=0$, then $\forall g \in \mathcal{C}_{0}\left(\mathbb{R}_{+}^{d}, \mathbb{R}\right),\left(\left\langle z_{m}, g\right\rangle\right)_{m \in \mathbb{N}^{*}}$ converges to 0 and $\left(\left\langle z_{m}, 1\right\rangle\right)_{m \in \mathbb{N}^{*}}$ also converges to 0 . This implies that $\left(z_{m}\right)_{m \in \mathbb{N}^{*}}$ converges weakly to the null measure.

The set $\bigcap_{r \in \mathbb{N}} \mathcal{K}_{\phi_{r}}\left(C_{r}\right)$ is hence relatively compact in $\left(\mathcal{M}_{F}\left(\mathbb{R}_{+}^{d}\right), w\right)$, and its adherence $\mathcal{K}_{L}^{2}$ is compact in $\left(\mathcal{M}_{F}\left(\mathbb{R}_{+}^{d}\right), w\right)$. Finally:

$$
\begin{aligned}
\mathbb{P}\left(\exists t \in[0, T], Z_{t}^{n} \notin \mathcal{K}_{L}^{2}\right) & \leq \sum_{r \in \mathbb{N}} \mathbb{P}\left(\exists t \in[0, T], Z_{t}^{n} \notin \mathcal{K}_{\phi_{r}}\left(C\left(\phi_{r}, T, r L \vee L, C_{0}\right)\right)\right) \\
& \leq \sum_{r \in \mathbb{N}^{*}} \mathrm{e}^{-n r L}+\mathrm{e}^{-n L} \leq \frac{\mathrm{e}^{-n L}\left(2-\mathrm{e}^{-n L}\right)}{1-\mathrm{e}^{-n L}}
\end{aligned}
$$

by Point (ii) of Lemma 4.6. This yields (4.12).

We can now conclude the proof of the exponential tightness. Let $N=N\left(T, L, C_{0}\right)$ be the positive constant given in Point (i) of Lemma 4.6. Consider the sets $\mathcal{K}_{L, N}^{1}$ and $\mathcal{K}_{L}^{2}$ given by Proposition 4.5. Let us define:

$$
\mathcal{K}_{L}=\mathcal{K}_{L, N}^{1} \cap\left\{z \in \mathbb{D}_{T} \text { such that } \forall t \in[0, T], z_{t} \in \mathcal{K}_{L}^{2}\right\}
$$

From Jakubowski [28] (Th. 1.7 and Lem. 3.3), the set $\mathcal{K}_{L}$ is relatively compact in $\mathbb{D}_{T}$. Since:

$$
\mathbb{P}\left(Z^{n} \notin \mathcal{K}_{L}\right) \leq \mathbb{P}\left(Z_{. \wedge \zeta_{N}^{n}}^{n} \notin \mathcal{K}_{L, N}^{1}\right)+\mathbb{P}\left(\exists t \in[0, T], Z_{t \wedge \zeta_{N}^{n}}^{n} \notin \mathcal{K}_{L}^{2}\right)+\mathbb{P}\left(\zeta_{N}^{n} \leq T\right)
$$

we have:

$$
\begin{aligned}
\limsup _{n \rightarrow+\infty} \frac{1}{n} \log \mathbb{P}\left(Z^{n} \notin \mathcal{K}_{L}\right) \leq & \max \left(\limsup _{n \rightarrow+\infty} \frac{1}{n} \log \mathbb{P}\left(Z_{. \wedge \zeta_{N}^{n}}^{n} \notin \mathcal{K}_{L, N}^{1}\right),\right. \\
& \left.\limsup _{n \rightarrow+\infty} \frac{1}{n} \log \mathbb{P}\left(\exists t \in[0, T], Z_{t \wedge \zeta_{N}^{n}}^{n} \notin \mathcal{K}_{L}^{2}\right), \limsup _{n \rightarrow+\infty} \frac{1}{n} \log \mathbb{P}\left(\zeta_{N}^{n} \leq T\right)\right) \leq-L,
\end{aligned}
$$

This concludes the proof.

\subsection{Lower bound of Theorem 4.3}

The proof of the lower bound relies on a change of probability and on the use of Girsanov theorem. We follow the work of Léonard [32], Kipnis Léonard [31], and thanks to a Riesz theorem, use the links between the trajectories in the domain of the action functional and some evolution equations obtained by perturbation of (1.2). We prove a local minoration for the neighborhood of trajectories associated to bounded perturbations. 
4.3.1. Representation of the paths of the action functional

Proposition 4.8. Under Assumptions 1, 2 and for $z \in \mathbb{D}_{T}$ such that $\mathcal{I}_{\xi_{0}}^{T}(z)<+\infty$ :

(i) there exists a function $h^{z} \in L^{\rho^{*}, z}$ satisfying $\forall\left(f:(a, s) \mapsto f_{s}(a)\right) \in \mathcal{C}_{b}^{1}\left(\mathbb{R}_{+}^{d} \times[0, T], \mathbb{R}\right)$ :

$$
\ell^{T}(f, z)=\int_{0}^{T} \int_{E} \psi(f)(a, u, s) h^{z}(a, u, s) m_{s}^{z, T}(d a, d u) \mathrm{d} s
$$

where $\ell^{T}(f, z)$ has been defined in (4.6).

(ii) Thus:

$$
\mathcal{I}^{f, T}(z)=\int_{0}^{T} \int_{E}\left[h^{z} \psi(f)-\rho(\psi(f))\right] \mathrm{d} m_{s}^{z, T} \mathrm{~d} s
$$

(iii) and $z$ is the solution of equation (4.7), which is a modified version of PDE (1.2) corresponding to perturbations in the jump rates of the original process.

Proof. From the definition of $\mathcal{I}_{\xi_{0}}^{T}$, we have for every $f \in \mathcal{C}_{b}^{1}\left(\mathbb{R}_{+}^{d} \times[0, T], \mathbb{R}\right)$ and every $\kappa \in \mathbb{R}^{*}$ :

$$
\mathcal{I}^{f / \kappa, T}(z)=\frac{1}{\kappa} \ell^{T}(f, z)-c^{T}\left(\frac{f}{\kappa}, z\right) \leq \mathcal{I}_{\xi_{0}}^{T}(z)<+\infty
$$

Using the notation $\tau(x)=\max (\rho(x), \rho(-x))=\rho(|x|)$ :

$$
\frac{1}{\kappa} \ell^{T}(f, z) \leq \mathcal{I}_{\xi_{0}}^{T}(z)+\int_{0}^{T} \int_{E} \rho\left(\frac{\psi(f)}{\kappa}\right) \mathrm{d} m_{s}^{z, T} \mathrm{~d} s \leq \mathcal{I}_{\xi_{0}}^{T}(z)+\int_{0}^{T} \int_{E} \tau\left(\frac{\psi(f)}{\kappa}\right) \mathrm{d} m_{s}^{z, T} \mathrm{~d} s .
$$

Choosing $\kappa=\|\psi(f)\|_{\rho, z}$ and $\kappa=-\|\psi(f)\|_{\rho, z}$, we obtain $\left|\ell^{T}(f, z)\right| \leq\left(1+\mathcal{I}_{\xi_{0}}^{T}(z)\right)\|\psi(f)\|_{\rho, z}$, from (4.4). The map $\psi(f) \mapsto \ell^{T}(f, z)$ is hence linear and continuous for the norm $\|\cdot\|_{\rho, z}$ on $\psi\left(\mathcal{C}_{b}^{1}\left(\mathbb{R}_{+}^{d} \times[0, T], \mathbb{R}\right)\right) \subset \mathcal{B}_{b}(E \times$ $[0, T], \mathbb{R})$. By Hahn-Banach's theorem, it is possible to extend this continuous linear form into a continuous linear form on $E^{\rho, z}(E \times[0, T])$. Thanks to Theorem 4.1, there exists a function $h^{z} \in L^{\rho^{*}, z}$ satisfying (4.22). From (4.22) and (4.5), we deduce (4.23).

For Point (iii), the fact that $z$ solves the perturbed PDE (4.7) is a consequence of (4.22) and (4.6).

The function $h^{z}$ given by the previous proposition is not unique, since it is only characterized by the functions of $\psi\left(\mathcal{C}_{b}^{1}\left(\mathbb{R}_{+}^{d} \times[0, T], \mathbb{R}\right)\right) \subset \mathcal{B}_{b}(E \times[0, T], \mathbb{R})$.

Lemma 4.9. We can choose $h^{z}$ such that for all $(a, s) \in \mathbb{R}_{+}^{d} \times[0, T], h^{z}(a, 0, s)$ depends only on $s$.

Proof. Let $h^{z} \in L^{\rho^{*}, z}$ be the perturbation given by Proposition 4.8. We are going to modify it on $\mathbb{R}_{+}^{d} \times\{0\} \times[0, T]$. $\forall f \in \mathcal{C}_{b}^{1}\left(\mathbb{R}_{+}^{d} \times[0, T], \mathbb{R}\right)$,

$$
\begin{gathered}
\int_{0}^{T} \int_{\mathbb{R}_{+}^{d}} f(0, s) h^{z}(a, 0, s) b(a) z_{s}(d a) \mathrm{d} s=\int_{0}^{T} \int_{\mathbb{R}_{+}^{d}} f(0, s) \bar{h}^{z}(s) b(a) z_{s}(d a) \mathrm{d} s \\
\text { with: } \bar{h}^{z}(s)=\frac{\int_{\mathbb{R}_{+}^{d}} h^{z}(a, 0, s) b(a) z_{s}(d a)}{\int_{\mathbb{R}_{+}^{d}} b(a) z_{s}(d a)}
\end{gathered}
$$


(If $\int_{\mathbb{R}_{+}^{d}} b(a) z_{s}(d a)=0$, the choice of $h^{z}$ on $\mathbb{R}_{+}^{d} \times\{0\} \times[0, T]$ is arbitrary). By the convexity of $\rho^{*}$ and by Jensen's inequality, $\forall \kappa>0$,

$$
\begin{aligned}
\int_{0}^{T} \int_{\mathbb{R}_{+}^{d}} \rho^{*}\left(\frac{\bar{h}^{z}(s)}{\kappa}\right) b(a) z_{s}(d a) \mathrm{d} s & =\int_{0}^{T} \int_{\mathbb{R}_{+}^{d}} \rho^{*}\left(\frac{\int_{\mathbb{R}_{+}^{d}} h^{z}(\alpha, 0, s) b(\alpha) z_{s}(d \alpha)}{\kappa \int_{\mathbb{R}_{+}^{d}} b(\alpha) z_{s}(d \alpha)}\right) b(a) z_{s}(d a) \mathrm{d} s \\
& \leq \int_{0}^{T} \int_{\mathbb{R}_{+}^{d}} \frac{1}{\int_{\mathbb{R}_{+}^{d}} b(\alpha) z_{s}(d \alpha)}\left[\int_{\mathbb{R}_{+}^{d}} \rho^{*}\left(\frac{h^{z}(\alpha, 0, s)}{\kappa}\right) b(\alpha) z_{s}(d a)\right] b(a) z_{s}(d a) \mathrm{d} s \\
& \leq \int_{0}^{T} \int_{\mathbb{R}_{+}^{d}} \rho^{*}\left(\frac{h^{z}(a, 0, s)}{\kappa}\right) b(a) z_{s}(d a) \mathrm{d} s
\end{aligned}
$$

and the perturbation obtained by replacing $h^{z}$ with $\bar{h}^{z}$ on $\mathbb{R}_{+}^{d} \times\{0\} \times[0, T]$ is again a function of $L^{\rho^{*}, z}$.

\subsubsection{Non-variational formula for $\mathcal{I}_{\xi_{0}}^{T}$}

In order to obtain a non-variational formula for the action functional, we are going to use () and approximate the perturbations $h^{z}$ by perturbations of the form $h_{m}=\mathrm{e}^{\psi\left(f_{m}\right)}-1$ with $\left(f_{m}\right)_{m \in \mathbb{N}^{*}}$ a sequence of $\mathcal{C}_{b}^{1}\left(\mathbb{R}_{+}^{d} \times[0, T], \mathbb{R}\right)$. The key result of this section is given in Proposition 4.12. To establish it, we will use the following lemma, which is a particular case of a result due to Ekeland Temam [14], Bishop Phelps [5], Israel [24]:

Lemma 4.10. Let $\mathfrak{F}$ be a closed subspace of a Banach space $\mathfrak{E}$ and let $\mathfrak{E}^{\prime}$ be the topological dual of $\mathfrak{E}$. Let $\Lambda$ be a convex continuous function from $\mathfrak{E}$ to $\mathbb{R}$ and let $\partial \Lambda(x)$ be its sub-differential in $x \in \mathfrak{E}$ defined by $\partial \Lambda(x)=\left\{\ell \in \mathfrak{E}^{\prime} \mid \forall y \in \mathfrak{E}, \Lambda(x)+\ell(y) \leq \Lambda(x+y)\right\}$. Let $\ell_{0} \in \mathfrak{E}^{\prime}$ such that:

$$
\exists c \in \mathbb{R}, \forall x \in \mathfrak{E}, \Lambda(x) \geq \ell_{0}(x)+c,
$$

then: $\forall \varepsilon>0, \exists x^{\prime} \in \mathfrak{F}, \exists \ell^{\prime} \in \partial \Lambda\left(x^{\prime}\right), \forall x \in \mathfrak{F},\left|\ell_{0}(x)-\ell^{\prime}(x)\right| \leq \varepsilon\|x\|$.

Definition 4.11. $\forall z \in \mathbb{D}_{T}, \forall g \in \mathcal{B}_{b}(E \times[0, T], \mathbb{R}), \forall h \in \mathcal{B}(E \times[0, T], \mathbb{R}), \forall \nu \in\left(L^{\infty}(E \times[0, T], \mathbb{R})\right)^{\prime}$,

$$
\begin{aligned}
& \Gamma_{\rho, z}(g):=\int_{0}^{T} \int_{E} \rho(g(a, u, s)) m_{s}^{z, T}(d a, d u) \mathrm{d} s \\
& \Gamma_{\rho, z}^{*}(h):=\sup _{f \in \mathcal{C}_{b}^{1}\left(\mathbb{R}_{+}^{d} \times[0, T], \mathbb{R}\right)}\left\{\int_{0}^{T} \int_{E} h \psi(f) \mathrm{d} m_{s}^{z, T} \mathrm{~d} s-\int_{0}^{T} \int_{E} \rho(\psi(f)) \mathrm{d} m_{s}^{z, T} \mathrm{~d} s\right\} \\
& \widetilde{\Gamma}_{\rho, z}^{*}(\nu):=\sup _{g \in \mathcal{B}_{b}(E \times[0, T], \mathbb{R})}\left\{\langle\nu, g\rangle-\Gamma_{\rho, z}(g)\right\} .
\end{aligned}
$$

By (4.5) and (4.23):

$$
\mathcal{I}_{\xi_{0}}^{T}(z)=\Gamma_{\rho, z}^{*}\left(h^{z}\right) .
$$

The main result of this Section 4.3.2 provides a non-variational formula for the action functional $\mathcal{I}_{\xi_{0}}^{T}(z)=$ $\Gamma_{\rho, z}^{*}\left(h^{z}\right)$, and an approximation result when we regularize $h^{z}$ :

Proposition 4.12. Under Assumptions 1, 2, for $z \in \mathbb{D}_{T}$ such that $\mathcal{I}_{\xi_{0}}^{T}(z)<+\infty$, and for the associated perturbation $h^{z} \in L^{\rho^{*}, z}$ (see Prop. 4.8), there exists a sequence $\left(h_{m}\right)_{m \in \mathbb{N}^{*}}$ of $\mathcal{C}_{b}(E \times[0, T], \mathbb{R})$ such that:

(i) $\forall m \in \mathbb{N}^{*}, \exists f_{m} \in \mathcal{C}_{b}^{1}\left(\mathbb{R}_{+}^{d} \times[0, T], \mathbb{R}\right), h_{m}=\mathrm{e}^{\psi\left(f_{m}\right)}-1$,

(ii) the sequence $\left(h_{m}\right)_{m \in \mathbb{N}^{*}}$ converges to $h^{z} \mathrm{~d} m_{s}^{z, T} \mathrm{~d}$ s-a.e.,

(iii) $\forall m \in \mathbb{N}^{*}, \Gamma_{\rho, z}^{*}\left(h_{m}\right)=\Gamma_{\rho^{*}, z}\left(h_{m}\right)$,

(iv) $h^{z} \in\left[-1,+\infty\left[\mathrm{d} m_{s}^{z, T} \mathrm{~d}\right.\right.$ s-a.e. and there exists $f^{z} \in \mathcal{B}\left(\mathbb{R}_{+}^{d} \times[0, T], \mathbb{R}\right)$ such that $\mathrm{d} m_{s}^{z, T} \mathrm{~d}$ s-a.e. on $\left\{h^{z}>-1\right\}$, $h^{z}(a, u, s)=\mathrm{e}^{\psi\left(f^{z}\right)(a, u, s)}-1$. 
(v) We have the following non-variational representation for the action functional:

$$
\begin{aligned}
\mathcal{I}_{\xi_{0}}^{T}(z) & =\Gamma_{\rho^{*}, z}\left(h^{z}\right)=\int_{0}^{T} \int_{E} \rho^{*}\left(h^{z}\right) \mathrm{d} m_{s}^{z, T} \mathrm{~d} s \\
& =\int_{0}^{T} \int_{E} \mathbf{1}_{\left\{h^{z}>-1\right\}}\left(h^{z} \psi\left(f^{z}\right)-\rho\left(\psi\left(f^{z}\right)\right)\right) \mathrm{d} m_{s}^{z, T} \mathrm{~d} s+\int_{0}^{T} \int_{E} \mathbf{1}_{\left\{h^{z}=-1\right\}} \mathrm{d} m_{s}^{z, T} \mathrm{~d} s .
\end{aligned}
$$

A difficulty lies in the fact that $\Gamma_{\rho, z}^{*}\left(h^{z}\right)$ and $\widetilde{\Gamma}_{\rho, z}^{*}\left(h^{z} \mathrm{~d} m_{s}^{z, T} \mathrm{~d} s\right)$ do not coincide a priori, since the sup in (4.26) and (4.27) are taken on different sets. This is due to the particular formulation of our problem which leads us to deal with births and deaths.

Before proving Proposition 4.12, we give a lemma for the functions of Definition 4.11.

Lemma 4.13. Under Assumptions 1, 2, for $z \in \mathbb{D}_{T}$,

(i) $\Gamma_{\rho, z}$ is convex continuous.

(ii) Let $f \in \mathcal{C}_{b}\left(\mathbb{R}_{+}^{d} \times[0, T], \mathbb{R}\right) . \partial \Gamma_{\rho, z}(\psi(f))$ is a singleton $\{\nu\}$ characterized by:

$$
\forall g \in \mathcal{B}_{b}(E \times[0, T], \mathbb{R}),\langle\nu, g\rangle=\int_{0}^{T} \int_{E} g\left(\mathrm{e}^{\psi(f)}-1\right) \mathrm{d} m_{s}^{z, T} \mathrm{~d} s .
$$

(iii) We have: $\forall h \in \mathcal{B}(E \times[0, T], \mathbb{R})$,

$$
\Gamma_{\rho, z}^{*}(h) \leq \widetilde{\Gamma}_{\rho, z}^{*}\left(h \mathrm{~d} m_{s}^{z, T} \mathrm{~d} s\right) \leq \Gamma_{\rho^{*}, z}(h) .
$$

Proof. The convexity of $\Gamma_{\rho, z}$ is a direct consequence of the convexity of $\rho$. For the continuity, we can see by dominated convergence that if $g \in \mathcal{B}_{b}(E \times[0, T], \mathbb{R})$ and if $\left(g_{q}\right)_{q \in \mathbb{N}^{*}}$ is a sequence that converges uniformly to $g$ in $\mathcal{B}_{b}(E \times[0, T], \mathbb{R})$, we have $\lim _{q \rightarrow+\infty} \Gamma_{\rho, z}\left(g_{q}\right)=\Gamma_{\rho, z}(g)$.

To characterize $\partial \Gamma_{\rho, z}(\psi(f))$, let $g \in \mathcal{B}_{b}(E \times[0, T], \mathbb{R})$,

$$
\begin{aligned}
\lim _{\varepsilon \rightarrow 0} \frac{\Gamma_{\rho, z}(\psi(f)+\varepsilon g)-\Gamma_{\rho, z}(\psi(f))}{\varepsilon} & =\lim _{\varepsilon \rightarrow 0} \int_{0}^{T} \int_{E}\left(\frac{\rho(\psi(f)+\varepsilon g)-\rho(\psi(f))}{\varepsilon}\right) \mathrm{d} m_{s}^{z, T} \mathrm{~d} s \\
& =\int_{0}^{T} \int_{E} g\left[\mathrm{e}^{\psi(f)}-1\right] \mathrm{d} m_{s}^{z, T} \mathrm{~d} s .
\end{aligned}
$$

This is obtained by taking the derivative under the integral. $\Gamma_{\rho, z}$ is hence Gâteau-differentiable in $\psi(f)$ and its sub-differential in $\psi(f)$ exists and is a singleton characterized by (4.30) (Ekeland Temam [14], Prop. 5.3 Chap. I).

The first inequality of (4.31) is a consequence of the inclusion $\psi\left(\mathcal{C}_{b}^{1}\left(\mathbb{R}_{+}^{d} \times[0, T], \mathbb{R}\right)\right) \subset \mathcal{B}_{b}(E \times[0, T], \mathbb{R})$. The second inequality is a consequence of the fact that $\forall(a, u, s) \in E \times[0, T], \forall g \in \mathcal{B}_{b}(E \times[0, T], \mathbb{R}), h(a, u, s) g(a, u, s)-$ $\rho(g(a, u, s)) \leq \rho^{*}(h(a, u, s))$.

We can now prove Proposition 4.12.

Proof of Proposition 4.12. We have $\mathcal{I}_{\xi_{0}}^{T}(z)=\Gamma_{\rho, z}^{*}\left(h^{z}\right)<+\infty$ by assumption, but we do not know whether $\widetilde{\Gamma}_{\rho, z}^{*}\left(h^{z} d m_{s}^{z, T} \mathrm{~d} s\right)<+\infty$. Since $h^{z} \in L^{\rho^{*}, z}$, we however know by (4.31) and by definition of the Orlicz norm $\|\cdot\|_{\rho^{*}, z}$ (4.4) that:

$$
\widetilde{\Gamma}_{\rho, z}^{*}\left(\frac{h^{z}}{\left\|h^{z}\right\|_{\rho^{*}, z}} \mathrm{~d} m_{s}^{z, T} \mathrm{~d} s\right) \leq \Gamma_{\rho^{*}, z}\left(\frac{h^{z}}{\left\|h^{z}\right\|_{\rho^{*}, z}}\right) \leq 1 .
$$

By definition of $\widetilde{\Gamma}_{\rho, z}^{*}$, and by $(4.32)$, it is possible to write: $\forall g \in \mathcal{B}_{b}(E \times[0, T], \mathbb{R})$,

$$
\Gamma_{\rho, z}(g) \geq \int_{0}^{T} \int_{E} \frac{h^{z}}{\left\|h^{z}\right\|_{\rho^{*}, z}} g \mathrm{~d} m_{s}^{z, T} \mathrm{~d} s-\widetilde{\Gamma}_{\rho, z}^{*}\left(\frac{h^{z}}{\left\|h^{z}\right\|_{\rho^{*}, z}} \mathrm{~d} m_{s}^{z, T} \mathrm{~d} s\right),
$$


and the condition (4.24) is satisfied. Applying Lemma 4.10 with the closed sub-space $\mathfrak{F}=$ $\operatorname{adh}\left(\psi\left(\mathcal{C}_{b}^{1}\left(\mathbb{R}_{+}^{d} \times[0, T], \mathbb{R}\right)\right)\right)$ of $L^{\infty}(E \times[0, T], \mathbb{R})$ gives: $\forall m \in \mathbb{N}^{*}, \exists \widetilde{f}_{m} \in \operatorname{adh}\left(\mathcal{C}_{b}^{1}\left(\mathbb{R}_{+}^{d} \times[0, T], \mathbb{R}\right)\right), \exists \widetilde{\nu}_{m} \in$ $\partial \Gamma_{\rho, z}\left(\psi\left(\widetilde{f}_{m}\right)\right), \forall g \in \mathcal{C}_{b}^{1}\left(\mathbb{R}_{+}^{d} \times[0, T], \mathbb{R}\right)$,

$$
\left|\int_{0}^{T} \int_{E} \psi(g) \frac{h^{z}}{\left\|h^{z}\right\|_{\rho^{*}, z}} \mathrm{~d} m_{s}^{z, T} \mathrm{~d} s-\left\langle\widetilde{\nu}_{m}, \psi(g)\right\rangle\right| \leq \frac{\|g\|_{\infty}}{2 m\left\|h^{z}\right\|_{\rho^{*}, z}} .
$$

By Point (ii) of Lemma 4.13 and setting $\widetilde{h}_{m}:=\left\|h^{z}\right\|_{\rho^{*}, z}\left(\mathrm{e}^{\psi\left(\tilde{f}_{m}\right)}-1\right)$ :

$$
\left|\int_{0}^{T} \int_{E} \psi(g)\left(\frac{h^{z}}{\left\|h^{z}\right\|_{\rho^{*}, z}}-\frac{\widetilde{h}_{m}}{\left\|h^{z}\right\|_{\rho^{*}, z}}\right) \mathrm{d} m_{s}^{z, T} \mathrm{~d} s\right| \leq \frac{\|g\|_{\infty}}{2 m\left\|h^{z}\right\|_{\rho^{*}, z}} .
$$

Since $\widetilde{f}_{m}$ is bounded and since $\mathrm{d} m_{s}^{z, T} \mathrm{~d} s$ is a finite measure, $\Gamma_{\rho^{*}, z}\left(\widetilde{h}_{m}\right)<+\infty$, implying by (4.31), that $\widetilde{\Gamma}_{\rho, z}^{*}\left(\widetilde{h}_{m} \mathrm{~d} m_{s}^{z, T} \mathrm{~d} s\right)<+\infty$. From the definition of $\widetilde{\Gamma}_{\rho, z}^{*}: \forall g \in \mathcal{B}_{b}(E \times[0, T], \mathbb{R})$,

$$
\Gamma_{\rho, z}(g) \geq \int_{0}^{T} \int_{E} \widetilde{h}_{m} g \mathrm{~d} m_{s}^{z, T} \mathrm{~d} s-\widetilde{\Gamma}_{\rho, z}^{*}\left(\widetilde{h}_{m} \mathrm{~d} m_{s}^{z, T} \mathrm{~d} s\right) .
$$

Applying the Lemma 4.10 and Point (ii) of Lemma 4.13, there exists a sequence $\left(f_{m}\right)_{m \in \mathbb{N}^{*}}$ of $\operatorname{adh}\left(\mathcal{C}_{b}^{1}\left(\mathbb{R}_{+}^{d} \times\right.\right.$ $[0, T], \mathbb{R})$ ), and a sequence of continuous and bounded perturbations $\left(h_{m}\right)_{m \in \mathbb{N}^{*}}$ defined by $h_{m}:=\mathrm{e}^{\psi\left(f_{m}\right)}-1$ such that: $\forall m \in \mathbb{N}^{*}, \forall g \in \mathcal{C}_{b}^{1}\left(\mathbb{R}_{+}^{d} \times[0, T], \mathbb{R}\right)$,

$$
\left|\int_{0}^{T} \int_{E} \psi(g)\left(h_{m}-\widetilde{h}_{m}\right) \mathrm{d} m_{s}^{z, T} \mathrm{~d} s\right| \leq \frac{\|g\|_{\infty}}{4 m} .
$$

Since each function $f_{m}$ is the uniform limit of a sequence $\left(f_{m, p}\right)_{p \in \mathbb{N}^{*}}$ of $\mathcal{C}_{b}^{1}\left(\mathbb{R}_{+}^{d} \times[0, T], \mathbb{R}\right), h_{m}$ is the limit, for the uniform norm and in $L^{1}\left(E \times[0, T], \mathrm{d} m_{s}^{z, T} \mathrm{~d} s\right)$ (since the measure $\mathrm{d} m_{s}^{z, T} \mathrm{~d} s$ is finite) of the sequence $h_{m, p}=\mathrm{e}^{\psi\left(f_{m, p}\right)}-1$. Replacing $h_{m}$ with $h_{m, p}$ for sufficiently large $p$, we obtain the existence of a sequence $\left(f_{m}\right)_{m \in \mathbb{N}^{*}}$ of $\mathcal{C}_{b}^{1}\left(\mathbb{R}_{+}^{d} \times[0, T], \mathbb{R}\right)$ satisfying:

$$
\left|\int_{0}^{T} \int_{E} \psi(g)\left(h_{m}-\widetilde{h}_{m}\right) \mathrm{d} m_{s}^{z, T} \mathrm{~d} s\right| \leq \frac{\|g\|_{\infty}}{2 m} .
$$

From (4.34) and (4.35): $\forall m \in \mathbb{N}^{*}, \forall g \in \mathcal{C}_{b}^{1}\left(\mathbb{R}_{+}^{d} \times[0, T], \mathbb{R}\right)$,

$$
\left|\int_{0}^{T} \int_{E} \psi(g)\left(h_{m}-h^{z}\right) \mathrm{d} m_{s}^{z, T} \mathrm{~d} s\right| \leq \frac{\|g\|_{\infty}}{m}
$$

with:

$$
\begin{aligned}
\int_{0}^{T} \int_{E} \psi(g)\left(h_{m}-h^{z}\right) \mathrm{d} m_{s}^{z, T} \mathrm{~d} s= & \int_{0}^{T} \int_{\mathbb{R}_{+}^{d}} g(0, s)\left(\mathrm{e}^{f_{m}(0, s)}-1-h^{z}(0,0, s)\right) b(a) z_{s}(d a) \mathrm{d} s \\
& -\int_{0}^{T} \int_{\mathbb{R}_{+}^{d}} g(a, s)\left(\mathrm{e}^{-f_{m}(a, s)}-1-h^{z}(a, 1, s)\right) d\left(a, z_{s} U(a)\right) z_{s}(d a) \mathrm{d} s .
\end{aligned}
$$


For $g \in \mathcal{C}_{K}^{1}\left(\left(\mathbb{R}_{+}^{*}\right)^{d} \times[0, T], \mathbb{R}\right)$, which satisfies $\forall s \in[0, T], g(0, s)=0$, we obtain the vague convergence of the sequence of measures $\left(h_{m} \mathrm{~d} m_{s}^{z, T} \mathrm{~d} s\right)_{m \in \mathbb{N}^{*}}$ to $h^{z} \mathrm{~d} m_{s}^{z, T} \mathrm{~d} s$ on $\left(\mathbb{R}_{+}^{*}\right)^{d} \times\{1\} \times[0, T]$. Hence, there exists a subsequence of $\left(\mathrm{e}^{-f_{m}}-1\right)_{m \in \mathbb{N}^{*}}$ that converges to $h^{z}(., 1,.) z_{s}(d a) \mathrm{d}$ s-a.e. on $\left(\mathbb{R}_{+}^{*}\right)^{d} \times[0, T]$. For $g \in \mathcal{C}_{b}^{1}\left(\mathbb{R}_{+}^{d} \times[0, T], \mathbb{R}\right)$, and for the previous sub-sequence of $\left(f_{m}\right)_{m \in \mathbb{N}^{*}}$ :

$$
\begin{aligned}
\mid \int_{0}^{T} g(0, s)\left[\int_{\mathbb{R}_{+}^{d}}\left(\mathrm{e}^{f_{m}(0, s)}-1-h^{z}(0,0, s)\right) b(a) z_{s}(d a)\right. \\
\left.\quad-\int_{\mathbb{R}_{+}^{d}} \mathbf{1}_{a=0}\left(\mathrm{e}^{-f_{m}(a, s)}-1-h^{z}(a, 1, s)\right) d\left(a, z_{s} U(a)\right) z_{s}(d a)\right] \mathrm{d} s \mid \leq \frac{C\|g\|_{\infty} .}{m} .
\end{aligned}
$$

If we prove that:

$$
\forall t \in[0, T],\left\langle z_{t}(d a), \mathbf{1}_{a=0}\right\rangle=0,
$$

then, $h^{z}(0,0, s)$ will be the ds-a.e. limit of a sub-sequence of $\left(\mathrm{e}^{f_{m}(0, s)}-1\right)_{m \in \mathbb{N}^{*}}$ and $h^{z}$ will be the $\mathrm{d} m_{s}^{z, T} \mathrm{~d} s$-a.e. limit of a sub-sequence of $\left(\mathrm{e}^{\psi\left(f_{m}\right)}-1\right)_{m \in \mathbb{N}^{*}}$. Let us prove (4.39). For $\varepsilon>0$, let $\phi_{\varepsilon} \in \mathcal{C}_{b}^{1}\left(\mathbb{R}_{+}^{d}, \mathbb{R}\right)$ be a non-negative function, bounded by 1 , with $\phi_{\varepsilon}(0)=1$ and $\phi_{\varepsilon}(a)=0$ for $|a| \geq \varepsilon$. For $t \in[0, T]$ and $f_{\varepsilon}(a, s):=\phi_{\varepsilon}(A(t, s, a))$ :

$$
\begin{aligned}
\left\langle z_{t}, \phi_{\varepsilon}\right\rangle=\left\langle z_{t}, f_{t}\right\rangle=\left\langle\xi_{0}, f_{0}\right\rangle+\int_{0}^{t} \int_{\mathbb{R}_{+}^{d}} \phi_{\varepsilon} & (A(t, s, 0))\left(1+h^{z}(a, 0, s)\right) b(a) z_{s}(d a) \mathrm{d} s \\
& -\int_{0}^{t} \int_{\mathbb{R}_{+}^{d}} \phi_{\varepsilon}(A(t, s, a))\left(1+h^{z}(a, 1, s)\right) d\left(a, z_{s} U(a)\right) z_{s}(d a) \mathrm{d} s .
\end{aligned}
$$

For $s<t$ and $a \in \mathbb{R}_{+}^{d}, \phi_{\varepsilon}(A(t, s, a)) \rightarrow 0$ when $\varepsilon \rightarrow 0$. Since $\phi_{\varepsilon}(A(t, s, 0))\left(1+h^{z}(a, 0, s)\right) b(a)$ and $\phi_{\varepsilon}(A(t, s, a))(1+$ $\left.h^{z}(a, 1, s)\right) d\left(a, z_{s} U(a)\right)$ are dominated by $\left(1+h^{z}(a, 0, s)\right) b(a)$ and $\left(1+h^{z}(a, 1, s)\right) d\left(a, z_{s} U(a)\right)$ which are integrable with respect to $z_{s}(d a) \mathrm{d} s$, the right hand side of (4.40) converges to 0 . The left hand side converges to $\left\langle z_{t}, \mathbf{1}_{a=0}\right\rangle$. This proves (4.39) and thus Points (i) and (ii).

Since $h_{m}=\mathrm{e}^{\psi\left(f_{m}\right)}-1, \rho^{*}\left(h_{m}\right)=\mathrm{e}^{\psi\left(f_{m}\right)} \psi\left(f_{m}\right)-\mathrm{e}^{\psi\left(f_{m}\right)}+1=h_{m} \psi\left(f_{m}\right)-\left(\mathrm{e}^{\psi\left(f_{m}\right)}-\psi\left(f_{m}\right)-1\right)$ :

$$
\begin{aligned}
\Gamma_{\rho^{*}, z}\left(h_{m}\right) & =\int_{0}^{T} \int_{E}\left[h_{m} \psi\left(f_{m}\right)-\rho\left(\psi\left(f_{m}\right)\right)\right] \mathrm{d} m_{s}^{z, T} \mathrm{~d} s \\
& \leq \sup _{f \in \mathcal{C}_{b}^{1}\left(\mathbb{R}_{+}^{d} \times[0, T]\right)}\left[\int_{0}^{T} \int_{E} h_{m} \psi(f) \mathrm{d} m_{s}^{z, T} \mathrm{~d} s-\Gamma_{\rho, z}(\psi(f))\right]=\Gamma_{\rho, z}^{*}\left(h_{m}\right) .
\end{aligned}
$$

From (4.31) and (4.41), $\Gamma_{\rho, z}^{*}\left(h_{m}\right)=\widetilde{\Gamma}_{\rho^{*}, z}\left(h_{m} \mathrm{~d} m_{s}^{z, T} \mathrm{~d} s\right)=\Gamma_{\rho^{*}, z}\left(h_{m}\right)$. This proves Point (iii).

Since $\left(h_{m}\right)_{m \in \mathbb{N}^{*}}$ converges $\mathrm{d} m_{s}^{z, T} \mathrm{~d} s$-a.e. to $h^{z}$ and since $\forall m \in \mathbb{N}^{*}, h_{m}>-1 \mathrm{~d} m_{s}^{z, T}$ ds-a.e., then $h^{z} \geq-1$ $\mathrm{d} m_{s}^{z, T} \mathrm{~d} s$-a.e. As $h^{z} \in L^{\rho^{*}, z} \subset L^{1}\left(E \times[0, T], \mathrm{d} m_{s}^{z, T} \mathrm{~d} s\right), h^{z}<+\infty \mathrm{d} m_{s}^{z, T} \mathrm{~d} s$-a.e. In the sequel, we have to separate the cases $h^{z}=-1$ and $h^{z}>-1$ to take the logarithm of $1+h^{z}$.

On $\left\{h^{z}>-1\right\}, \psi\left(f_{m}\right)(a, u, s)=\log \left(h_{m}(a, u, s)+1\right)$ converges $\mathrm{d} m_{s}^{z, T} \mathrm{~d} s$-a.e. to $\psi\left(f^{z}\right)(a, u, s):=\log \left(h^{z}(a, u, s)+\right.$ 1). The sequence $h^{z} \psi\left(f_{m}\right)-\rho\left(\psi\left(f_{m}\right)\right)$ thus converges to $h^{z} \psi\left(f^{z}\right)-\rho\left(\psi\left(f^{z}\right)\right)$, and the latter is non-negative:

$$
\begin{aligned}
0 \leq \rho^{*}\left(h^{z}\right) & =\mathrm{e}^{\psi\left(f^{z}\right)} \psi\left(f^{z}\right)-\mathrm{e}^{\psi\left(f^{z}\right)}+1 \\
& =\left(\mathrm{e}^{\psi\left(f^{z}\right)}+1\right) \psi\left(f^{z}\right)-\left(\mathrm{e}^{\psi\left(f^{z}\right)}-\psi\left(f^{z}\right)-1\right)=h^{z} \psi\left(f^{z}\right)-\rho\left(\psi\left(f^{z}\right)\right) .
\end{aligned}
$$

On $\left\{h^{z}=-1\right\}, \psi\left(f_{m}\right)$ diverges to $-\infty$ and $h^{z} \psi\left(f_{m}\right)-\rho\left(\psi\left(f_{m}\right)\right)=-\mathrm{e}^{\psi\left(f_{m}\right)}+1$ converges to 1 . 
Let us define the sequence $\left(\breve{f}_{m}\right)_{m \in \mathbb{N}^{*}}$ by:

$$
\psi\left(\breve{f}_{m}\right)=\psi\left(f_{m}\right) \mathbf{1}_{\left\{h^{z} \psi\left(f_{m}\right)-\rho\left(\psi\left(f_{m}\right)\right) \geq 0, h^{z}>-1\right\}}+\psi\left(f_{m}\right) \mathbf{1}_{\left\{\psi\left(f_{m}\right) \leq 0, h^{z}=-1\right\}} .
$$

The non-negative sequence $\left(h^{z} \psi\left(\breve{f}_{m}\right)-\rho\left(\psi\left(\breve{f}_{m}\right)\right)\right)_{m \in \mathbb{N}^{*}}$ converges to $\left[h^{z} \psi\left(f^{z}\right)-\rho\left(\psi\left(f^{z}\right)\right)\right] \mathbf{1}_{\left\{h^{z}>-1\right\}}+\mathbf{1}_{\left\{h^{z}=-1\right\}}=$ $\rho^{*}\left(h^{z}\right)$. Thus, $\Gamma_{\rho^{*}, z}\left(h^{z}\right)<+\infty$. Else, by Fatou lemma and (4.42):

$$
\begin{aligned}
\Gamma_{\rho, z}^{*}\left(h^{z}\right)= & \sup _{f \in \mathcal{C}_{b}^{1}\left(\mathbb{R}_{+}^{d} \times[0, T], \mathbb{R}\right)}\left\{\int_{0}^{T} \int_{E}\left(h^{z} \psi(f)-\rho(\psi(f))\right) \mathrm{d} m_{s}^{z, T} \mathrm{~d} s\right\} \\
\geq \liminf _{m \rightarrow+\infty} \int_{0}^{T} \int_{E}\left(h^{z} \psi\left(\breve{f}_{m}\right)-\rho\left(\psi\left(\breve{f}_{m}\right)\right)\right) \mathrm{d} m_{s}^{z, T} \mathrm{~d} s \geq & \int_{0}^{T} \int_{E} \liminf _{m \rightarrow+\infty}\left(h^{z} \psi\left(\breve{f}_{m}\right)-\rho\left(\psi\left(\breve{f}_{m}\right)\right)\right) \mathrm{d} m_{s}^{z, T} \mathrm{~d} s \\
& =\int_{0}^{T} \int_{E} \rho^{*}\left(h^{z}\right) \mathrm{d} m_{s}^{z, T} \mathrm{~d} s=\Gamma_{\rho^{*}, z}\left(h^{z}\right)=+\infty, \quad
\end{aligned}
$$

which contradicts the assumption $\Gamma_{\rho, z}^{*}\left(h^{z}\right)<+\infty$. Integrating (4.42) gives the last equality of (4.29). Moreover Point (iii) of Lemma 4.13 and (4.44) imply the first equality of (4.29).

Remark 4.14. When $h^{z}$ is bounded, we can choose the functions $h_{m}$ bounded by $\left\|h^{z}\right\|_{\infty}$. Indeed, if we regularize the functions $f_{m}$ in the neighborhood of the points where $\psi\left(f_{m}\right) \geq \log \left(\left\|h^{z}\right\|_{\infty}+1\right)$, we obtain a new sequence $\left(\widehat{h}_{m}=\mathrm{e}^{\psi\left(\widehat{f}_{m}\right)}-1\right)_{m \in \mathbb{N}^{*}}$ bounded by $\left\|h^{z}\right\|_{\infty}$ and satisfying $\left|h^{z}-\widehat{h}_{m}\right|=\left|\mathrm{e}^{\psi\left(f^{z}\right)}-\mathrm{e}^{\psi\left(\widehat{f}_{m}\right)}\right| \leq$ $\left|\mathrm{e}^{\psi\left(f^{z}\right)}-\mathrm{e}^{\psi\left(f_{m}\right)}\right|=\left|h^{z}-h_{m}\right|$.

\subsubsection{Local lower bound}

The proof of the local lower bound enounced in Theorem 4.3 relies on a change of probability, and on the use of Girsanov theorem. Let $P^{n}=\mathcal{L}\left(Z^{n}\right)$. It is a probability law on $\mathbb{D}_{T}$. Let $z \in \mathbb{D}_{T}$ be such that $\mathcal{I}_{\xi_{0}}^{T}(z)<+\infty$. Our purpose is to construct a sequence of probability measures $\left(P^{n n}\right)_{n \in \mathbb{N}^{*}}$ on $\mathbb{D}_{T}$ that are absolutely continuous with respect to $\left(P^{n}\right)_{n \in \mathbb{N}^{*}}$ and which can be interpreted as a recentering on $z$. To achieve this, we use the perturbed equation (4.7) satisfied by $z$. The use of the Girsanov theorem is allowed if $h^{z}$ has a particular form and proper regularities. Since $h^{z} \in L^{\rho^{*}, z}$ is not a priori bounded or differentiable, we use regularized perturbations $h_{m}$ of the form given in Point 1 of Proposition 4.12. It is hence natural to introduce the sequence $\left(z^{m}\right)_{m \in \mathbb{N}^{*}}$ of the solutions of (4.7) perturbed by the $h_{m}$ and to apply a recentering associated with these $z^{m}$. Unfortunately, we can prove the convergence of $\left(z^{m}\right)_{m \in \mathbb{N}^{*}}$ to $z$ only in the case where $h^{z}$ is bounded. The case where $h^{z}$ is unbounded remains open. Thus, we will be lead to introduce the additional assumption $h^{z} \in L^{\infty}(E \times[0, T], \mathbb{R})$.

The following result implies the lower bound of Theorem 4.3:

Proposition 4.15. Under Assumptions 1, 2, 5, for $z \in \mathbb{D}_{T}$ such that $\mathcal{I}_{\xi_{0}}^{T}(z)<+\infty$ and $h^{z} \in L^{\infty}(E \times[0, T], \mathbb{R})$, we have for any open subset $O$ of $\mathbb{D}_{T}$ containing $z$ :

$$
\liminf _{n \rightarrow+\infty} \frac{1}{n} \log \mathbb{P}\left(Z^{n} \in O\right) \geq-\mathcal{I}_{\xi_{0}}^{T}(z),
$$

(in the case where $\mathcal{I}_{\xi_{0}}^{T}(z)=+\infty$, the result is automatically satisfied).

First, we complete the approximation result of Proposition 4.12 by showing that every path $z$ of the domain of the action functional $\mathcal{I}_{\xi_{0}}^{T}$ associated to a bounded $h^{z}$ can be approximated by a sequence of paths $\left(z^{m}\right)_{m \in \mathbb{N}^{*}}$ associated to perturbations $h_{m}$ of the form $h_{m}=\mathrm{e}^{\psi\left(f_{m}\right)}-1$ with $f_{m} \in \mathcal{C}_{b}^{1}\left(\mathbb{R}_{+}^{d} \times[0, T], \mathbb{R}\right)$. 
Proposition 4.16. Under Assumptions 1, 2, for $z \in \mathbb{D}_{T}$ such that $\mathcal{I}_{\xi_{0}}^{T}(z)<+\infty$ and $h^{z} \in L^{\rho^{*}, z} \cap L^{\infty}(E \times$ $[0, T], \mathbb{R})$, there exists a sequence $\left(f_{m}\right)_{m \in \mathbb{N}^{*}}$ of $\mathcal{C}_{b}^{1}\left(\mathbb{R}_{+}^{d} \times[0, T], \mathbb{R}\right)$ such that the sequence defined by $h_{m}=\mathrm{e}^{\psi\left(f_{m}\right)}-1$ satisfies:

(i) $\left(h_{m}\right)_{m \in \mathbb{N}^{*}}$ converges to $h^{z} d m_{s}^{z, T} \mathrm{~d}$ s-a.e. and (4.36) is fulfilled.

(ii) For every $m \in \mathbb{N}^{*}$, the evolution equation: $\forall f \in \mathcal{C}_{b}^{1}\left(\mathbb{R}_{+}^{d} \times \mathbb{R}_{+}, \mathbb{R}\right)$,

$$
\left\langle y_{t}, f_{t}\right\rangle=\left\langle\xi_{0}, f_{0}\right\rangle+\int_{0}^{t}\left\langle y_{s}, v \nabla_{a} f_{s}+\frac{\partial f_{s}}{\partial s}\right\rangle \mathrm{d} s+\int_{0}^{t} \int_{E} \exp \left(\psi\left(f_{m}\right)\right) \psi(f) \mathrm{d} m_{s}^{y, T} \mathrm{~d} s
$$

admits a unique solution in $y$, which is denoted by $z^{m} \in \mathcal{C}\left([0, T], \mathcal{M}_{F}\left(\mathbb{R}_{+}^{d}\right)\right)$.

(iii) The sequence $\left(z^{m}\right)_{m \in \mathbb{N}^{*}}$ converges to $z \in\left(\mathcal{C}\left([0, T],\left(\mathcal{M}_{F}\left(\mathbb{R}_{+}^{d}\right),\|\cdot\|_{T V}\right)\right)\right.$, $\left.\|\cdot\|_{\infty}\right)$.

(iv) We have $\mathcal{I}_{\xi_{0}}^{T}\left(z^{m}\right)=\int_{0}^{T} \int_{E}\left(h_{m} \psi\left(f_{m}\right)-\rho\left(\psi\left(f_{m}\right)\right)\right) \mathrm{d} m_{s}^{z^{m}, T} \mathrm{~d} s=\int_{0}^{T} \int_{E} \rho^{*}\left(h_{m}\right) \mathrm{d} m_{s}^{z^{m}, T} \mathrm{~d} s$ and:

$$
\lim _{m \rightarrow+\infty} \mathcal{I}_{\xi_{0}}^{T}\left(z^{m}\right)=\mathcal{I}_{\xi_{0}}^{T}(z)
$$

Proof. Point (i) is a consequence of Proposition 4.12. By Remark 4.14:

$$
\left\langle z_{t}^{m}, 1\right\rangle \leq\left\langle\xi_{0}, 1\right\rangle+\int_{0}^{t} \int_{\mathbb{R}_{+}^{d}} \mathrm{e}^{f_{m}(0, s)} b(a) z_{s}^{m}(d a) \mathrm{d} s \leq\left\langle\xi_{0}, 1\right\rangle \mathrm{e}^{\left(\left\|h^{z}\right\|_{\infty}+1\right) \bar{b} t},
$$

we can define $A_{T}=\max \left(\sup _{t \in[0, T]}\left\langle z_{t}, 1\right\rangle, \sup _{t \in[0, T]}\left\langle z_{t}^{m}, 1\right\rangle, m \in \mathbb{N}^{*}\right)$ which exists and is finite and independent from $m$.

Existence and uniqueness of the solution $z^{m} \in \mathcal{C}\left([0, T], \mathcal{M}_{F}\left(\mathbb{R}_{+}^{d}\right)\right)$ of $(4.46)$ is an adaptation of the results of Section 3.2 (the birth and death rates are perturbed by continuous bounded factors and Assumptions 2 still hold).

Let $t \in[0, T], \phi \in \mathcal{C}_{b}^{1}\left(\mathbb{R}_{+}^{d}, \mathbb{R}\right)$ such that $\|\phi\|_{\infty} \leq 1$ and $f:(a, s) \in \mathbb{R}_{+}^{d} \times[0, T] \mapsto f(a, s)=\phi(A(t, s, a)) \in \mathbb{R}$.

$$
\left|\left\langle z_{t}-z_{t}^{m}, \phi\right\rangle\right|=\left|\int_{0}^{t} \int_{E}\left(1+h^{z}\right) \psi(f) \mathrm{d} m_{s}^{z, T} \mathrm{~d} s-\int_{0}^{t} \int_{E}\left(1+h_{m}\right) \psi(f) \mathrm{d} m_{s}^{z^{m}, T} \mathrm{~d} s\right| \leq A+B
$$

where by (4.36):

$$
A:=\left|\int_{0}^{t} \int_{E}\left(h^{z}-h_{m}\right) \psi(f) \mathrm{d} m_{s}^{z, T} \mathrm{~d} s\right| \leq \frac{1}{m}
$$

and, by Remark 4.14 and computations similar to (3.1):

$$
B:=\left|\int_{0}^{t} \int_{E}\left(1+h_{m}\right) \psi(f)\left[\mathrm{d} m_{s}^{z, T}-\mathrm{d} m_{s}^{z^{m}, T}\right] \mathrm{d} s\right| \leq C\left(\left\|h^{z}\right\|_{\infty}, T\right) \int_{0}^{t}\left\|z_{s}-z_{s}^{m}\right\|_{T V} \mathrm{~d} s
$$

We deduce from (4.48), (4.49), (4.50) that:

$$
\left|\left\langle z_{t}-z_{t}^{m}, \phi\right\rangle\right| \leq \frac{1}{m}+C\left(\left\|h^{z}\right\|_{\infty}, T\right) \int_{0}^{t}\left\|z_{s}-z_{s}^{m}\right\|_{T V} \mathrm{~d} s .
$$

Since every function $\phi \in \mathcal{C}_{b}\left(\mathbb{R}_{+}^{d}, \mathbb{R}_{+}\right)$bounded by 1 can be written as the pointwise limit of a sequence of $\mathcal{C}_{b}^{1}\left(\mathbb{R}_{+}^{d}, \mathbb{R}_{+}\right)$bounded by 1 , we obtain by taking the supremum in the left hand side:

$$
\sup _{u \in[0, t]}\left\|z_{u}-z_{u}^{m}\right\|_{T V} \leq \frac{1}{m}+C\left(\left\|h^{z}\right\|_{\infty}, T\right) \int_{0}^{t} \sup _{u \in[0, s]}\left\|z_{u}-z_{u}^{m}\right\|_{T V} \mathrm{~d} s
$$


and by Gronwall Lemma: $\sup _{t \in[0, T]}\left\|z_{t}-z_{t}^{m}\right\|_{T V} \leq C\left(\left\|h^{z}\right\|_{\infty}, T\right) / m$. Hence:

$$
\forall \varepsilon>0, \exists m_{0}=m_{0}(\varepsilon, T) \in \mathbb{N}^{*}, \forall m \geq m_{0}, \sup _{t \in[0, T]}\left\|z_{t}-z_{t}^{m}\right\|_{T V} \leq \varepsilon
$$

and $\left(z^{m}\right)_{m \in \mathbb{N}^{*}}$ converges to $z$ in $\mathcal{C}\left([0, T],\left(\mathcal{M}_{F}\left(\mathbb{R}_{+}^{d}\right),\|\cdot\|_{T V}\right)\right)$ uniformly in $t \in[0, T]$.

By an adaptation of the proof of Point 3 of Proposition 4.12:

$$
\Gamma_{\rho^{*}, z^{m}}\left(h_{m}\right)=\Gamma_{\rho, z^{m}}^{*}\left(h_{m}\right) .
$$

By (4.5), (4.23), (4.31) and (4.53) $\mathcal{I}_{\xi_{0}}^{T}\left(z^{m}\right)=\Gamma_{\rho, z^{m}}^{*}\left(h_{m}\right)=\Gamma_{\rho^{*}, z^{m}}\left(h_{m}\right)$, which proves the first part of (iv). Finally let us prove (4.47):

$$
\begin{aligned}
& \leq \leq\left|\mathcal{I}_{\xi_{0}}^{T}\left(z^{m}\right)-\mathcal{I}_{\xi_{0}}^{T}(z)\right| \leq A+B \\
A= & \left|\int_{0}^{T} \int_{E} \rho^{*}\left(h_{m}\right) \mathrm{d} m_{s}^{z, T} \mathrm{~d} s-\int_{0}^{T} \int_{E} \rho^{*}\left(h^{z}\right) \mathrm{d} m_{s}^{z, T} \mathrm{~d} s\right| \\
B= & \left|\int_{0}^{T} \int_{E} \rho^{*}\left(h_{m}\right) \mathrm{d} m_{s}^{z^{m}, T} \mathrm{~d} s-\int_{0}^{T} \int_{E} \rho^{*}\left(h_{m}\right) \mathrm{d} m_{s}^{z, T} \mathrm{~d} s\right| .
\end{aligned}
$$

By (4.52): $\forall m \geq m_{0}, B \leq \int_{0}^{T} \rho^{*}\left(\left\|h_{m}\right\|_{\infty}\right)\left(\bar{b}+\bar{d}\left(1+A_{T}\right)+L_{d} \bar{U} A_{T}\right)\left\|z_{s}-z_{s}^{m}\right\|_{T V} \mathrm{~d} s \leq C\left(\left\|h^{z}\right\|_{\infty}, T\right) \varepsilon$. Since $\left(h_{m}\right)_{m \in \mathbb{N}^{*}}$ converges $\mathrm{d} m_{s}^{z, T} \mathrm{~d} s$-a.e. to $h^{z}$, and since these functions are bounded, we have by dominated convergence that $\lim _{m \rightarrow+\infty} \Gamma_{\rho^{*}, z}\left(h_{m}\right)=\Gamma_{\rho^{*}, z}\left(h^{z}\right)=\mathcal{I}_{\xi_{0}}^{T}(z)$, and $\lim _{m \rightarrow+\infty} A=0$. This concludes the proof of (4.47).

Thanks to Propositions 4.12 and 4.16 and under the Assumptions of Proposition 4.15, we can restrict the study of the local minoration to "regular" paths:

Assumption 6. We consider a path $z$ belonging to the domain of the action functional $\mathcal{I}_{\xi_{0}}^{T}$ and such that $h^{z}=\mathrm{e}^{\psi\left(f^{z}\right)}-1$ with $f^{z} \in \mathcal{C}_{b}^{1}\left(\mathbb{R}_{+}^{d} \times \mathbb{R}_{+}, \mathbb{R}\right)$. Then:

$$
\mathcal{I}_{\xi_{0}}^{T}(z)=\int_{0}^{T} \int_{E}\left(h^{z} \psi\left(f^{z}\right)-\rho\left(\psi\left(f^{z}\right)\right)\right) \mathrm{d} m_{s}^{z, T} \mathrm{~d} s .
$$

We construct the change of probability corresponding to a centering around a such path $z$.

Proposition 4.17. For a path $z$ as in Assumption 6, the following SDE on $[0, T]$ :

$$
\begin{aligned}
D_{t}^{n}=1+\int_{0}^{t} \int_{\mathcal{E}} D_{s_{-}}^{n} \mathbf{1}_{\left\{i \leq N_{t}^{n}\right\}}\left[\left(\mathrm{e}^{f^{z}(0, s)}-1\right) \mathbf{1}_{\left\{0 \leq \theta \leq m_{1}\left(s, Z_{s_{-}}^{n}, i\right)\right\}}\right. & \\
& \left.+\left(\mathrm{e}^{f^{z}\left(\pi_{i}\left(Z_{s_{-}}^{n}\right), s\right)}-1\right) \mathbf{1}_{\left\{m_{1}\left(s, Z_{s_{-}}^{n}, i\right)<\theta \leq m_{2}\left(s, Z_{s_{-}}^{n}, i\right)\right\}}\right] \widetilde{Q}(d s, d i, d \theta)
\end{aligned}
$$

admits a unique solution $\left(D_{t}^{n}\right)_{t \in[0, T]}$ which is a $P^{n}$-exponential martingale:

$$
\begin{aligned}
D_{t}^{n}= & \exp \left(n \left[\left\langle Z_{t}^{n}, f_{t}^{z}\right\rangle-\left\langle Z_{0}^{n}, f_{0}^{z}\right\rangle-\int_{0}^{t} \int_{\mathbb{R}_{+}^{d}}\left(v(a) \nabla_{a} f_{s}^{z}(a)+\frac{\partial f_{s}^{z}}{\partial s}(a)\right) Z_{s}^{n}(d a) \mathrm{d} s\right.\right. \\
& \left.\left.-\int_{0}^{t} \int_{E} \psi\left(f^{z}\right) \mathrm{d} m_{s}^{Z^{n}, T} \mathrm{~d} s-\int_{0}^{t} \int_{E} \rho\left(\psi\left(f^{z}\right)\right) \mathrm{d} m_{s}^{Z^{n}, T} \mathrm{~d} s\right]\right) .
\end{aligned}
$$


Under the probability $P^{\prime n}:=D_{T}^{n} \cdot P^{n}$, the compensator of the random measure $Q$ is:

$$
\widehat{q}(d s, d i, d \theta)=1_{\left\{i \leq N_{t}^{n}\right\}}\left[\mathrm{e}^{f^{z}(0, s)} \mathbf{1}_{\left\{0 \leq \theta \leq m_{1}\left(s, Z_{s_{-},}^{n}, i\right)\right\}}+\mathrm{e}^{f^{z}\left(\pi_{i}\left(Z_{s_{-}}^{n}\right), s\right)} \mathbf{1}_{\left\{m_{1}\left(s, Z_{s_{-}}^{n}, i\right)<\theta \leq m_{2}\left(s, Z_{s_{-}}^{n}, i\right)\right\}}\right] \mathrm{d} s n(d i) \mathrm{d} \theta .
$$

Proof. We wish to perturb the birth and death rate of the particles, so that the new rates are multiplied by $1+h^{z}(a, u, s)=\mathrm{e}^{\psi\left(f^{z}\right)(a, u, s)}, u \in\{0,1\}$. This is why we introduce SDE (4.55). For the proofs, we refer to Jacod and Shiryaev [25], Theorems 3.24 p. 159 and 5.19 p. 181.

Proposition 4.18. Under Assumptions 1, 2, 5, for $z \in \mathbb{D}_{T}$ satisfying Assumption 6 and under $\left(P^{\prime n}\right)_{n \in \mathbb{N}^{*}}$ of Proposition 4.17 , the sequence $\left(Z^{n}\right)_{n \in \mathbb{N}^{*}}$ converges in probability to $z$.

Proof. Birth and death rates are perturbed by continuous bounded functions:

$$
b(a) \leftrightarrow \mathrm{e}^{f^{z}(0, s)} b(a), \quad d\left(a, Z_{s}^{n} U(a)\right) \leftrightarrow \mathrm{e}^{-f^{z}(a, s)} d\left(a, Z_{s}^{n} U(a)\right) .
$$

Assumptions 2 are still satisfied. We can then prove, with proofs similar to the ones of Section 3 (Cor. 3.3), that $Z^{n}$ converges weakly to $z$ in $P^{\prime n}$-probability.

We are now able to prove the lower bound announced in (4.45).

Proof of Proposition 4.15. We have $P^{n}(O)=P^{\prime n}(O) \mathbb{E}^{P^{\prime n}}\left(\mathbf{1}_{O} \frac{d P^{n}}{d P^{\prime n}} / P^{\prime n}(O)\right)$ (these terms may all be equal to zero). By Jensen's inequality and the definition of $D_{T}^{n}$ :

$$
\liminf _{n \rightarrow \infty} \frac{1}{n} \log P^{n}(O) \geq \liminf _{n \rightarrow \infty} \frac{1}{n} \log P^{\prime n}(O)+\liminf _{n \rightarrow \infty} \mathbb{E}^{P^{\prime n}}\left(-\frac{\mathbf{1}_{O}}{P^{\prime n}(O)} \frac{1}{n} \log D_{T}^{n}\right) .
$$

By Proposition 4.18, $\lim _{n \rightarrow+\infty} \log P^{\prime n}(O) \rightarrow 0$, which implies: $\liminf _{n \rightarrow \infty} \frac{1}{n} \log P^{\prime n}(O)=0$. From (4.56), from Proposition 4.18, from the fact that $z$ is continuous and that $h^{z}$ and $f^{z}$ are continuous and bounded, we obtain the following convergence in probability:

$$
\begin{aligned}
\lim _{n \rightarrow \infty} \frac{1}{n} \log D_{T}^{n}= & \lim _{n \rightarrow \infty}\left[\left\langle Z_{t}^{n}, f_{t}^{z}\right\rangle-\left\langle Z_{0}^{n}, f_{0}^{z}\right\rangle-\int_{0}^{t} \int_{\mathbb{R}_{+}^{d}}\left(v(a) \nabla_{a} f_{s}^{z}(a)+\frac{\partial f_{s}^{z}}{\partial s}\right) Z_{s}^{n}(d a) \mathrm{d} s\right. \\
& \left.-\int_{0}^{t} \int_{E} \psi\left(f^{z}\right) \mathrm{d} m_{s}^{Z^{n}, T} \mathrm{~d} s-\int_{0}^{t} \int_{E} \rho\left(\psi\left(f^{z}\right)\right) \mathrm{d} m_{s}^{Z^{n}, T} \mathrm{~d} s\right] \\
= & \int_{0}^{t} \int_{E} h^{z} \psi\left(f^{z}\right) \mathrm{d} m_{s}^{z, T} \mathrm{~d} s-\int_{0}^{t} \int_{E} \rho\left(\psi\left(f^{z}\right)\right) \mathrm{d} m_{s}^{z, T} \mathrm{~d} s=\mathcal{I}_{\xi_{0}}^{T}(z),
\end{aligned}
$$

by (4.22) and (4.29). Since: $\left|\frac{1}{n} \log D_{T}^{n}\right| \leq C\left(T,\left\|f^{z}\right\|_{\infty}\right)\left(1+\sup _{t \in[0, T]}\left\langle Z_{t}^{n}, 1\right\rangle^{2}\right)$, we obtain, by Point 2 of Assumptions 3 and by Proposition 2.7:

$$
\limsup _{n \rightarrow \infty} \mathbb{E}^{P^{\prime n}}\left(\frac{\mathbf{1}_{O}}{P^{\prime n}(O)} \frac{1}{n} \log D_{T}^{n}\right) \leq \int_{0}^{t} \int_{E}\left[h^{z} \psi\left(f^{z}\right)-\rho\left(\psi\left(f^{z}\right)\right)\right] \mathrm{d} m^{z, T} \mathrm{~d} s=\mathcal{I}_{\xi_{0}}^{T}(z) .
$$

This concludes the proof of Theorem 4.3.

\section{Application to problems of exit of domains}

We deduce from Theorem 4.3 some estimates on times of exit of domains. The computations that we present are inspired by results obtained by Freidlin and Ventzell [18], Dembo and Zeitouni [12] (Sect. 5) in finite 
dimension, and Da Prato and Zabczyk [40] (Chap. 12). We adapt these works to the case of our measure-valued processes.

This allows us to control the probability of exit of a tube around the deterministic solution (Sect. 5.1) and to give estimates for the exit time of a neighborhood of an attractive domain of the stationary solution of the deterministic limit solution in the particular case of logistic age-structured populations.

In the proofs, the distance $\mathcal{W}_{1}$ appears naturally since the consideration of an age-structure leads us to introduce functions $f$ of $\mathcal{C}_{b}^{1}\left(\mathbb{R}_{+}^{d}, \mathbb{R}\right)$ such that $\nabla_{a} f$ is bounded. These functions are Lipschitz continuous. The total variation norm arises since we do not know if the perturbations $h^{z}$ are Lipschitz continuous. However, under the constraint that $z \in \mathfrak{G}$, these perturbations remain bounded. The difficulty will be to deal with these two distances. We have:

$$
\forall \mu, \nu \in \mathcal{M}_{F}\left(\mathbb{R}_{+}^{d}\right), \mathcal{W}_{1}(\mu, \nu) \leq\|\mu-\nu\|_{T V} .
$$

We denote by $B_{T V}(\mu, r)$ (resp. $\left.B_{\mathcal{W}_{1}}(\mu, r)\right)$ the $\|\cdot\|_{T V}$-ball (resp. the $\mathcal{W}_{1}$-ball) of radius $r>0$, centered in $\mu \in \mathcal{M}_{F}\left(\mathbb{R}_{+}^{d}\right) . d_{\mathbb{D}, T}$ is the Skorohod distance on $\mathbb{D}_{T}$ (see. $[4,25]$ ).

\subsection{Exit times from tubes and pits}

Proposition 5.1. Let $R>0, \delta>0$ and let $K(R)$ and $K(R)_{\delta}$ be the sets defined by:

$$
K(R)=\left\{z \in \mathbb{D}_{T}, \quad \mathcal{I}_{\xi_{0}}^{T}(z) \leq R\right\} \quad \text { and } \quad K(R)_{\delta}=\left\{z \in \mathbb{D}_{T}, \quad \exists z^{\prime} \in K(R), d_{\mathbb{D}, T}\left(z, z^{\prime}\right)<\delta\right\} .
$$

Under Assumptions 1, 2, 5:

(i) $\forall R>0, \forall \delta>0, \forall \varepsilon>0, \exists n_{0} \in \mathbb{N}^{*}, \forall n \geq n_{0}, \mathbb{P}\left(Z^{n} \in K(R)_{\delta}\right) \geq 1-\mathrm{e}^{-n(R-\varepsilon)}$.

(ii) $\forall z \in \mathfrak{G}, \forall \delta>0, \forall \varepsilon>0, \exists n_{0} \in \mathbb{N}^{*}, \forall n \geq n_{0}, \mathbb{P}\left(d_{\mathbb{D}, T}\left(Z^{n}, z\right)<\delta\right) \geq \mathrm{e}^{-n\left[\mathcal{I}_{\xi_{0}}^{T}(z)+\varepsilon\right]}$.

Proof. For $R>0, \delta>0$ and $z \in \mathbb{D}_{T}, K(R)_{\delta}^{c}$ is closed in $\mathbb{D}_{T}$ and $\left\{y \in \mathbb{D}_{T} \mid d_{\mathbb{D}, T}(z, y)<\delta\right\}$ is open. The results are then consequences of Theorem 4.3.

Remark 5.2. Since $\left\{y \mid d_{\mathbb{D}, T}(y, \xi)<\delta\right\} \cap \mathfrak{G} \neq \emptyset$, the lower bound of (4.10) provides information for the times of exit from tubes centered on the solution $\xi$ of (1.2).

\subsection{Exit times from the neighborhood of a stationary state of the Logistic age-structured population}

Recall the logistic population structured by a scalar age presented in the introduction. Let us introduce the probability $\Pi\left(a_{1}, a_{2}\right)$ that an individual of age $a_{1}$ lives until age $a_{2}$ and the net reproduction rate $R_{0}$.

$$
\Pi\left(a_{1}, a_{2}\right)=\exp \left(-\int_{a_{1}}^{a_{2}} d(\alpha) \mathrm{d} \alpha\right) \quad \text { and } \quad R_{0}=\int_{0}^{+\infty} b(a) \Pi(0, a) \mathrm{d} a .
$$

Assumption 7. In the following, we assume that $R_{0}>1$. (Hence $\bar{b}>\underline{d}$ since: $\bar{b} / \underline{d}=\int_{0}^{+\infty} \bar{b} \mathrm{e}^{-\underline{d} a} \mathrm{~d} a \geq R_{0}>1$.)

There exists a unique classical solution to PDE (1.5) that describes the large population limit of the microscopic process and we have an explicit expression (Prop. 5.3). Under Assumption 7, the behaviour of the solution of (1.5) is moreover known: there exists a unique nontrivial stable stationary solution $\widehat{m}(a)$ to which every solution starting from a nonzero initial condition converges (Prop. 5.4). These are results due to [6,22,34,51].

The estimates given by Section 5.1 tell us that the microscopic stochastic process behaves as its deterministic approximation on compact time intervals, and we prove in Proposition 5.5 that for sufficiently large $T>0$ and $n, Z_{T}^{n}$ finds itself with "large probability" in a neighborhood of the stationary solution $\widehat{m}(a) \mathrm{d} a$.

However, the same Proposition 5.5 indicates that in the long time, and for a given integer $n,\left(Z_{t}^{n}\right)_{t \in \mathbb{R}_{+}}$ does not converge to $\widehat{m}(a) \mathrm{d} a$, but to the null measure. The realizations of $\left(Z_{t}^{n}\right)_{t \in \mathbb{R}_{+}}$almost surely leave the neighborhood of $\widehat{m}(a) \mathrm{d} a$ to drive the population to extinction. 
Proposition 5.3. Under Assumptions 1, 2, 4, if $\exists m_{0} \in \mathcal{C}_{b}^{1}\left(\mathbb{R}_{+}, \mathbb{R}_{+}\right), \xi_{0}(d a)=m_{0}(a) \mathrm{d} a$ :

(i) $\left(Z^{n}\right)_{n \in \mathbb{N}^{*}}$ converges in probability in $\mathbb{D}$ to the weak measure solution $\left(\xi_{t}\right)_{t \in \mathbb{R}_{+}}$of (1.5).

(ii) For every $t \in \mathbb{R}_{+}, \xi_{t}$ is absolutely continuous with respect to the Lebesgue measure on $\mathbb{R}_{+}$. The family of the densities $(m(., t))_{t \in \mathbb{R}_{+}}$is the unique classical solution of $(1.5)$. It belongs to $\mathcal{C}^{1}\left(\mathbb{R}_{+}^{2}, \mathbb{R}_{+}\right) \cap L^{1}\left(\mathbb{R}_{+}^{2}, \mathbb{R}_{+}\right)$and its explicit expression is given by: $\forall a \in \mathbb{R}_{+}, \forall t \in \mathbb{R}_{+}$,

$$
m(a, t)=\frac{M_{0} \nu(a, t)}{1+M_{0} \int_{0}^{t} \int_{0}^{+\infty} \nu(\alpha, s) \mathrm{d} \alpha \mathrm{d} s}, \quad \text { with: } \nu(a, t)=\left\{\begin{array}{cll}
\frac{m_{0}(a-t)}{M_{0}} \Pi(a-t, a) & \text { if } & a \geq t, \\
B(t-a) \Pi(a, 0) & \text { if } \quad a<t,
\end{array}\right.
$$

$\Pi$ being defined in (5.2) and for $g^{* n}$ the $n$ th-convolution of the function $g$ with itself:

$$
B(t)=B_{0} *\left(\sum_{n=0}^{+\infty} g^{* n}(t)\right), B_{0}(t)=\mathbf{1}_{t \geq 0} \int_{0}^{+\infty} b(a+t) \frac{m_{0}(a)}{M_{0}} \Pi(a, a+t) \mathrm{d} a, g(a)=b(a) \Pi(0, a) \mathbf{1}_{a \geq 0} .
$$

Proof. Existence and uniqueness of a weak function solution are particular cases of Corollary 3.3 and Proposition 3.4. The computations of $[6,22,34,51]$ tell us that $(1.5)$ admits a classical solution.

This provides regularity information and an explicit expression of the function solution of (1.2) in this case.

Proposition 5.4. Under Assumptions 1, 2, 4, \%:

(i) The following equation: $1=\int_{0}^{+\infty} \mathrm{e}^{-\lambda_{1} a} b(a) \Pi(0, a) \mathrm{d} a$, admits a unique solution $\lambda_{1}>0$.

(ii) The solution $m(a, t)$ of (1.5) has the following long-time behaviour:

$$
\lim _{t \rightarrow+\infty} m(a, t)=\frac{\lambda_{1} \mathrm{e}^{-\lambda_{1} a} \Pi(0, a)}{\eta \int_{0}^{+\infty} \mathrm{e}^{-\lambda_{1} \alpha} \Pi(0, \alpha) \mathrm{d} \alpha}=: \widehat{m}(a),
$$

the convergence being uniform in age on bounded intervals of $\mathbb{R}_{+}$.

(iii) $\widehat{m}$ is exponentially asymptotically stable and $\forall \xi_{0} \in \mathcal{M}_{F}\left(\mathbb{R}_{+}^{d}\right), \forall R>0, \exists T_{1}=T_{1}\left(R, \xi_{0}\right) \geq 0, \forall t \geq$ $T_{1}, \mathcal{W}_{1}\left(\xi_{t}, \widehat{\xi}\right)<R$, where $\widehat{\xi}(d a)=\widehat{m}(a) \mathrm{d} a$.

Proof. These assertions are proved in [6,34,51] and Proposition 5.6.4 of [47].

Proposition 5.5. Under Assumptions 1, 2, 4, if $\exists m_{0} \in \mathcal{C}_{b}^{1}\left(\mathbb{R}_{+}, \mathbb{R}_{+}\right), \xi_{0}(d a)=m_{0}(a) \mathrm{d} a$ :

(i) For all $n \in \mathbb{N}^{*}, \mathbb{P}\left(\exists t \in \mathbb{R}_{+},\left\langle Z_{t}^{n}, 1\right\rangle=0\right)=1$.

(ii) $\forall \varepsilon>0, \forall \varsigma>0, \exists T_{1}=T_{1}(\varsigma)>0, \exists n_{1}=n_{1}\left(\varepsilon, T_{1}(\varsigma)\right) \in \mathbb{N}^{*}, \forall n \geq n_{1}, \mathbb{P}\left(\mathcal{W}_{1}\left(Z_{T_{1}}^{n}, \widehat{\xi}\right)>\varsigma\right) \leq \varepsilon$.

Proof. Let $n \in \mathbb{N}^{*}$ be fixed. The idea in the proof of Point (i) is to dominate stochastically $\left(\left\langle Z_{t}^{n}, 1\right\rangle\right)_{t \in \mathbb{R}_{+}}$by the following process $\left(Y_{t}^{n}\right)_{t \in \mathbb{R}_{+}}$with values in $(1 / n) \mathbb{N}$ :

$$
\begin{aligned}
Y_{t}^{n}= & \left\langle Z_{0}^{n}, 1\right\rangle+\int_{0}^{t} \int_{\mathcal{E}} \mathbf{1}_{\left\{i \leq n Y_{s_{-}}^{n}\right\}} \frac{1}{n}\left(\mathbf{1}_{0 \leq \theta<b\left(\pi_{i}\left(Z_{s_{-}}^{n}\right)\right)}-\mathbf{1}_{b\left(\pi_{i}\left(Z_{s_{-}}^{n}\right)\right) \leq \theta<b\left(A_{i}\left(Z_{s_{-}}^{n}\right)\right)+\underline{d}+\eta Y_{s_{-}}^{n}}\right) Q(d s, d i, d \theta) \\
& +\int_{0}^{t} \int_{\mathcal{E}} \frac{1}{n}\left[\mathbf{1}_{\left\{Y_{s_{-}}^{n}=0, i \in \llbracket 1, n \rrbracket, \theta \in[0,1]\right\}}+\mathbf{1}_{\left\{i \leq n Y_{s_{-}}^{n}\right\}} \mathbf{1}_{0 \leq \theta<\bar{b}-b\left(\pi_{i}\left(Z_{s_{-}}^{n}\right)\right)}\right] Q^{\prime}(d s, d i, d \theta) .
\end{aligned}
$$

$Q$ is the Poisson point measure introduced in Definition 2.4 and $Q^{\prime}$ is an independent copy. The second term of (5.4) implies that 0 is not an absorbing state for $Y^{n}$ and, when $Y_{t}^{n}>0$, completes the birth events so that the birth rate is $\bar{b}$. Let us prove that $Y^{n}$ is positive recurrent. This process is irreducible. Its generator $\mathcal{A}$ is 
defined by: $\forall F \in \mathcal{C}\left((1 / n) \mathbb{N}, \mathbb{R}_{+}\right), \forall i \in \mathbb{N}$,

$$
\begin{aligned}
\mathcal{A} F(i / n)= & \mathbf{1}_{i>0}\left\{\left(F\left(\frac{i+1}{n}\right)-F\left(\frac{i}{n}\right)\right) \bar{b} i+\left(F\left(\frac{i-1}{n}\right)-F\left(\frac{i}{n}\right)\right)\left(\underline{d}+\eta \frac{i}{n}\right) i\right\} \\
& +n\left(F\left(\frac{1}{n}\right)-F(0)\right) \mathbf{1}_{i=0} .
\end{aligned}
$$

If we choose for $F$ the Lyapounov function $V^{n}: i / n \mapsto i / n$, then $\forall i \in \mathbb{N}$,

$$
\mathcal{A} V^{n}\left(\frac{i}{n}\right)=\frac{i}{n}\left[\bar{b}-\underline{d}-\eta \frac{i}{n}\right] \mathbf{1}_{i>0}+\mathbf{1}_{i=0} \leq-V^{n}\left(\frac{i}{n}\right)+\left(\frac{(\bar{b}-\underline{d})^{2}}{4 \eta}+1\right)
$$

since $\bar{b}-\underline{d}-\eta i / n<-1$ for $i>n(\bar{b}+1-\underline{d}) / \eta$, and

$$
\sup _{0<i \leq \frac{n(\bar{b}+1-\underline{d})}{\eta}}\left\{\frac{i}{n}\left[\bar{b}-\underline{d}-\eta \frac{i}{n}\right]\right\} \leq \frac{(\bar{b}-\underline{d})^{2}}{4 \eta} .
$$

The inequality (5.6) is of the form $\forall i \in \mathbb{N}, \mathcal{A} V^{n}\left(\frac{i}{n}\right) \leq-c V^{n}\left(\frac{i}{n}\right)+c^{\prime}$. A criterium of Meyn and Tweedie [38], Ths. 6.1 and 7.1) then entails that $Y^{n}$ is positive recurrent.

A sufficient condition to prove Point (i) is that $\mathbb{P}\left(\exists t \in \mathbb{R}_{+}, Y_{t}^{n}=0\right)=1$. Let $C>1$ and

$$
\tau_{C}=\inf \left\{t>0, Y_{t}^{n}=0 \text { or } Y_{t}^{n}=C / n\right\} .
$$

Since $\left(Y_{t}^{n}\right)_{t \in \mathbb{R}_{+}}$is positive recurrent, $\mathbb{P}_{C / n}\left(Y_{\tau_{C}}^{n}=0\right)>0$ (under $\mathbb{P}_{C / n}, Y^{n}$ starts from $C / n$ ).

$$
\begin{aligned}
\mathbb{P}\left(\exists t \in \mathbb{R}_{+}, Y_{t}^{n}=0\right) & =\mathbb{P}\left(Y_{\tau_{C}}^{n}=0\right)+\mathbb{P}\left(Y_{\tau_{C}}^{n}=C / n \text { and } \exists t \in \mathbb{R}_{+}, Y_{t+\tau_{C}}^{n}=0\right) \\
& =\mathbb{P}\left(Y_{\tau_{C}}^{n}=0\right)+\mathbb{P}\left(Y_{\tau_{C}}^{n}=C / n\right) \mathbb{P}_{C / n}\left(\exists t \in \mathbb{R}_{+}, Y_{t}^{n}=0\right), \text { by the strong Markov property } \\
& =\mathbb{P}\left(Y_{\tau_{C}}^{n}=0\right)+\mathbb{P}\left(Y_{\tau_{C}}^{n}=C / n\right)\left[\mathbb{P}_{C / n}\left(Y_{\tau_{C}}^{n}=0\right)+\mathbb{P}_{C / n}\left(Y_{\tau_{C}}^{n}=C / n \text { and } \exists t \in \mathbb{R}_{+}, Y_{t+\tau_{C}}^{n}=0\right)\right] \\
& =\mathbb{P}\left(Y_{\tau_{C}}^{n}=0\right)+\mathbb{P}\left(Y_{\tau_{C}}^{n}=C / n\right)\left[\sum_{\ell=0}^{+\infty} \mathbb{P}_{C / n}\left(Y_{\tau_{C}}^{n}=0\right) \mathbb{P}_{C / n}\left(Y_{\tau_{C}}^{n}=C / n\right)^{\ell}\right], \text { by recursion } \\
& =\mathbb{P}\left(Y_{\tau_{C}}^{n}=0\right)+\mathbb{P}\left(Y_{\tau_{C}}^{n}=C / n\right) \frac{\mathbb{P}_{C / n}\left(Y_{\tau_{C}}^{n}=0\right)}{1-\mathbb{P}_{C / n}\left(Y_{\tau_{C}}^{n}=C / n\right)}=\mathbb{P}\left(Y_{\tau_{C}}^{n}=0\right)+\mathbb{P}\left(Y_{\tau_{C}}^{n}=C / n\right)=1 .
\end{aligned}
$$

This concludes the proof. Point (ii) is a consequence of Proposition 5.1.

In order to illustrate these Propositions, we have simulated the stochastic process $Z^{n}$ for different values of $n$ and have represented in Figure 3 the evolution of the sizes of the corresponding populations. For increasing $n$, we can see that the fluctuations around the equilibrium value given by the deterministic approximation, $\int_{\mathbb{R}_{+}} \widehat{m}(a) \mathrm{d} a$, are of decreasing amplitudes, and that the extinction times are increasing. For $n=5$ or $n=10$, these times are already very large and cannot be observed on simulations.

For numerous applications (see $[37,47]$, Chap. 6 for instance), it is of great interest to evaluate the time during which the microscopic process stays in the neighborhood of the stationary stable solution $\widehat{m}(a) \mathrm{d} a$ of its deterministic approximation. This is obtained in Propositions 5.6 and 5.11. We will prove that this time is exponential in $n$. This explains why the extinction time in the simulations of Figure 3 increases so fast.

Assumption 8. Assume that the functions $a \mapsto b(a)$ and $a \mapsto d(a)$ are Lipschitz continuous from $\mathbb{R}_{+}$to $\mathbb{R}_{+}$, with constants $L_{b}$ and $L_{d}$. 

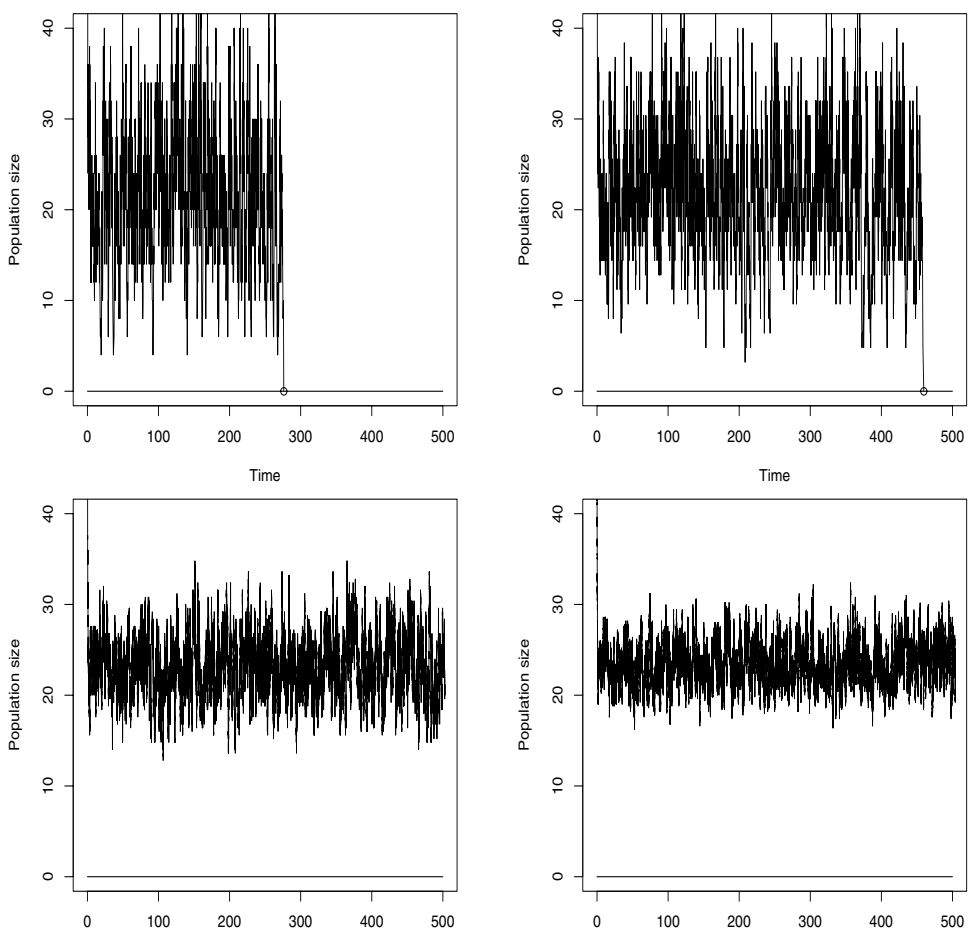

FiguRE 3. Simulation of a logistic age-structured population under different renormalizations $(n=1, n=1.25, n=5, n=10)$.

We assume that the limit $\xi_{0}$ of $\left(Z_{0}^{n}\right)_{n \in \mathbb{N}^{*}}$ in $\left(\mathcal{M}_{F}\left(\mathbb{R}_{+}\right)\right.$, w) is absolutely continuous with respect to the Lebesgue measure, with a density $m_{0} \in \mathcal{C}^{1}\left(\mathbb{R}_{+}, \mathbb{R}_{+}\right) \cap L^{1}\left(\mathbb{R}_{+}, \mathbb{R}_{+}\right)$.

Let $0<\gamma<\langle\widehat{\xi}, 1\rangle$. We study the problem of exit from the domain $B_{\mathcal{W}_{1}}(\widehat{\xi}, \gamma)$ using the results of Theorem 4.3 . Results are stated in Propositions 5.6 and 5.11. Notice that for this choice of $\gamma$, this neighborhood does not contain the null measure. The exit time of $B_{\mathcal{W}_{1}}(\widehat{\xi}, \gamma)$ is:

$$
\mathcal{T}^{n}=\inf \left\{t \geq 0, \mid Z_{t}^{n} \notin B_{\mathcal{W}_{1}}(\widehat{\xi}, \gamma)\right\}
$$

For $R>0$ and $\mathfrak{G}$ defined in (4.9):

$$
\begin{aligned}
\bar{V} & =\inf \left\{\mathcal{I}_{\xi_{0}}^{T}(z) \mid T>0, z \in \mathbb{D}_{T} \cap \mathfrak{G}, z_{0}=\widehat{\xi}, z_{T} \notin B_{\mathcal{W}_{1}}(\widehat{\xi}, \gamma)\right\} \\
\underline{V}(R) & =\inf \left\{\mathcal{I}_{z_{0}}^{T}(z) \mid T>0, z \in \mathbb{D}_{T}, \mathcal{W}_{1}\left(z_{0}, \widehat{\xi}\right)<R, z_{T} \notin B_{\mathcal{W}_{1}}(\widehat{\xi}, \gamma)\right\} \\
\underline{V} & =\lim _{R \rightarrow 0} \underline{V}(R) .
\end{aligned}
$$

When the set in (5.8) (resp. (5.9)) is empty, $\bar{V}$ (resp. $\underline{V}(R)$ ) is infinite. The estimates given in the following sections (Props. 5.6 and 5.11) are then automatically satisfied. Notice that $\underline{V}$ is well defined in $\mathbb{R}_{+} \cup\{+\infty\}$ : $(\underline{V}(R))_{R>0}$ is an increasing sequence when $R \downarrow 0$ and:

$$
\underline{V}:=\lim _{R \rightarrow 0} \underline{V}(R) \leq \bar{V}
$$


Notation. Recall that $\xi$ is the solution of (1.2) starting from $\xi_{0} \in \mathcal{M}_{F}\left(\mathbb{R}_{+}\right)$, which is the limit of $Z_{0}^{n}$ for the weak convergence. Until the end of this section, we will consider $\xi_{0} \in B_{\mathcal{W}_{1}}(\widehat{\xi}, \gamma)$. We denote by $\xi^{\mu_{0}} \in \mathcal{C}\left(\mathbb{R}_{+}, \mathcal{M}_{F}\left(\mathbb{R}_{+}\right)\right)$ the solution of (1.2) starting from the initial condition $\mu_{0} \in \mathcal{M}_{F}\left(\mathbb{R}_{+}\right)$.

For $z \in \mathbb{D}_{T}$ such that $\mathcal{I}_{\xi_{0}}^{T}(z)<+\infty$ with $T>0$, there exists $h^{z} \in L^{\rho^{*}, z}$ such that $z$ is the solution of (4.7) perturbed by $h^{z}$. We denote by $z^{\mu_{0}}$ the solution of (4.7) perturbed by $h^{z}$ and starting from $\mu_{0} \in \mathcal{M}_{F}\left(\mathbb{R}_{+}\right)$.

\subsubsection{Upper bound of the exit time}

Proposition 5.6. Let $\xi_{0} \in B_{\mathcal{W}_{1}}(\widehat{\xi}, \gamma)$. Under Assumptions 1, 2, 5, 7, 8:

$$
\limsup _{n \rightarrow+\infty} \frac{1}{n} \log \mathbb{E}\left(\mathcal{T}^{n}\right) \leq \bar{V}
$$

The following corollary, obtained by the Markov inequality, gives us an interpretation of this result: the exit time $\mathcal{T}^{n}$ is upper bounded by $\mathrm{e}^{n(\bar{V}+\delta)}$, for any $\delta>0$, up to an exponentially small probability.

Corollary 5.7. Let $\xi_{0} \in B_{\mathcal{W}_{1}}(\widehat{\xi}, \gamma)$. Under Assumptions 1, 2, 5, 7, $8: \forall \delta>0, \exists C>0$,

$$
\limsup _{n \rightarrow+\infty} \frac{1}{n} \log \mathbb{P}\left(\mathcal{T}^{n} \geq \mathrm{e}^{n(\bar{V}+\delta)}\right) \leq-C .
$$

The proof of Proposition 5.6 relies on the following lemmas. Lemma 5.8, proved in the end of this section, establishes a result of continuity with respect to the initial condition. Lemma 5.9 shows that $z \mapsto \mathcal{I}_{\xi_{0}}^{T}(z)$ vanishes only at $\xi$. Its proof stands in [20] page 490. Notice that the results given by these lemmas use the total variation norm. Indeed, we do not know if the perturbations $h^{z}$ are Lipschitz continuous. However, under the constraint that $z \in \mathfrak{G}$ in the definition of $\bar{V}$ and in the lower bound of Theorem 4.3, these perturbations remain bounded.

Lemma 5.8. Let $T>0$, let $z \in \mathbb{D}_{T} \cap \mathfrak{G}$ with initial condition $z_{0} \in \mathcal{M}_{F}\left(\mathbb{R}_{+}\right)$.

(i) $\forall \varepsilon>0, \exists \delta>0, \forall \mu_{0} \in B_{T V}\left(z_{0}, \delta\right), \sup _{t \in[0, T]}\left\|z_{t}^{\mu_{0}}-z_{t}\right\|_{T V}<\varepsilon$.

(ii) The map $\mu_{0} \mapsto \mathcal{I}_{\mu_{0}}^{T}\left(z^{\mu_{0}}\right)$ from $\left(\mathcal{M}_{F}\left(\mathbb{R}_{+}\right),\|\cdot\|_{T V}\right)$ into $\mathbb{R}_{+}$is continuous at point $\mu_{0}=z_{0}$.

Lemma 5.9. Let $T>0$. We have $\mathcal{I}_{\xi_{0}}^{T}(z)=0$ if and only if $z=\xi$, the unique weak measure solution of (1.4)(1.3) starting from $\xi_{0}$.

Proof Proposition 5.6. Let $z \in \mathbb{D}\left(\mathbb{R}_{+}, \mathcal{M}_{F}\left(\mathbb{R}_{+}\right)\right)$such that $z_{0}=\widehat{\xi}, \exists T>0, z_{T} \notin B_{\mathcal{W}_{1}}(\widehat{\xi}, \gamma),\left(z_{t}\right)_{t \in[0, T]} \in \mathfrak{G}$. We do this assumption since the upper bound (5.12) is based on the lower bound in (4.10). In order to establish the Proposition 5.6, it is sufficient to prove that:

$$
\limsup _{n \rightarrow+\infty} \frac{1}{n} \log \mathbb{E}\left(\mathcal{T}^{n}\right) \leq \mathcal{I}_{\widehat{\xi}}^{T}(z) .
$$

The left hand side does not depend on $z$. Taking the infimum in $z$ in the right hand side gives (5.12) by (5.8). In order to obtain (5.14), we establish an upper bound of the form:

$$
\forall n \in \mathbb{N}^{*}, \forall k \in \mathbb{N}^{*}, \mathbb{P}\left(\mathcal{T}^{n}>k C(z, T)\right) \leq(p(z, T))^{k},
$$

where $C(z, T)$ is a non-negative constant and $p(z, T)$ is a constant that belongs to ]0,1[. For this purpose, we lower bound the probability $\mathbb{P}\left(\mathcal{T}^{n} \leq k C(z, T)\right)$ by the probability that $\left(Z_{t}^{n}\right)_{t \in[0, k C(z, T)]}$ goes through the neighborhood of $z_{T} \in \mathcal{M}_{F}\left(\mathbb{R}_{+}\right)$situated outside of $B_{\mathcal{W}_{1}}(\widehat{\xi}, \gamma)$.

Step 1. We begin with bounding below the exit probability of $B_{\mathcal{W}_{1}}(\widehat{\xi}, \gamma)$ when the initial condition is "near" $\widehat{\xi}$, by using the path $z$. 
Let $\delta:=\inf \left\{\mathcal{W}_{1}\left(z_{T}, y\right), y \in B_{\mathcal{W}_{1}}(\widehat{\xi}, \gamma)\right\}$. Since $z_{T} \notin B_{\mathcal{W}_{1}}(\widehat{\xi}, \gamma), \delta>0$. Let us consider the perturbed evolution equation (4.7) satisfied by $z$ starting from $\widehat{\xi}$ and let $\mu_{0} \in \mathcal{M}_{F}\left(\mathbb{R}_{+}\right)$.

By Point (i) of Lemma 5.8, $\left.\forall \delta_{1} \in\right] 0, \delta\left[, \exists \delta_{2}>0,\left[\left\|\mu_{0}-\widehat{\xi}\right\|_{T V}<\delta_{2}\right] \Rightarrow\left[\left\|z_{T}^{\mu_{0}}-z_{T}\right\|_{T V}<\delta-\delta_{1}\right]\right.$. We deduce by the triangular inequality: $\forall \mu_{0} \in B_{T V}\left(\widehat{\xi}, \delta_{2}\right)$,

$$
\inf _{y \in B \mathcal{W}_{1}(\widehat{\xi}, \gamma)} \mathcal{W}_{1}\left(z_{T}^{\mu_{0}}, y\right) \geq \inf _{y \in B \mathcal{W}_{1}(\widehat{\xi}, \gamma)} \mathcal{W}_{1}\left(z_{T}, y\right)-\left\|z_{T}^{\mu_{0}}-z_{T}\right\|_{T V} \geq \delta-\left(\delta-\delta_{1}\right)=\delta_{1}>0,
$$

and thus $z_{T}^{\mu_{0}} \notin B_{\mathcal{W}_{1}}(\widehat{\xi}, \gamma)$.

By Point (ii) of Lemma 5.8:

$$
\forall \varepsilon>0, \exists \delta_{0}>0, \forall \mu_{0} \in B_{T V}\left(\widehat{\xi}, \delta_{0}\right),\left|\mathcal{I}_{\mu_{0}}^{T}\left(z^{\mu_{0}}\right)-\mathcal{I}_{\widehat{\xi}}^{T}(z)\right| \leq \varepsilon .
$$

Assume that $\xi_{0} \in B_{T V}\left(\widehat{\xi}, \delta_{0} \wedge \delta_{2}\right)$. We can lower bound the probability of exit of $B_{\mathcal{W}_{1}}(\widehat{\xi}, \gamma)$ by the probability that the path stays in a tube of radius $\delta_{1}$ around $z^{\xi_{0}}: \forall \varepsilon>0, \exists n_{0} \in \mathbb{N}^{*}, \forall n \geq n_{0}$,

$$
\mathbb{P}\left(\mathcal{T}^{n} \leq T\right) \geq \mathbb{P}\left(d_{\mathbb{D}, T}\left(Z^{n}, z^{\xi_{0}}\right)<\delta_{1}\right) \geq \mathrm{e}^{-n\left(\mathcal{I}_{\xi_{0}}^{T}\left(z^{\xi_{0}}\right)+\varepsilon\right)} \geq \mathrm{e}^{-n\left(\mathcal{I}_{\tilde{\xi}}^{T}(z)+2 \varepsilon\right)},
$$

by Proposition 5.1 and by (5.16).

Step 2. We now consider an initial condition $\xi_{0} \in B_{\mathcal{W}_{1}}(\widehat{\xi}, \gamma)$ satisfying Assumption 8. By Proposition 3.4, the marginals $\xi_{t}$ of the solution of (1.2) are absolutely continuous with respect to the Lebesgue measure. By Point (iii) of Proposition 5.4,

$$
\exists T_{1}=T_{1}\left(\delta_{0} \wedge \delta_{2}, \widehat{\xi}, \gamma\right) \geq 0,\left\|\xi_{T_{1}}-\widehat{\xi}\right\|_{T V}<\delta_{0} \wedge \delta_{2}
$$

Let $\delta_{3}=\inf _{t \in\left[0, T_{1}\right]}\left(\gamma-\mathcal{W}_{1}\left(\xi_{t}, \widehat{\xi}\right)\right)>0$ (the deterministic path $\xi$ remains in $\left.B_{\mathcal{W}_{1}}(\widehat{\xi}, \gamma)\right)$. Since $\left(Z_{T_{1}}^{n}\right)_{n \in \mathbb{N}^{*}}$ converges in probability to $\xi_{T_{1}} \in B_{T V}\left(\widehat{\xi}, \delta_{0} \wedge \delta_{2}\right)$, we obtain from (5.17), and from the strong Markov property:

$$
\mathbb{P}\left(\mathcal{T}^{n} \leq T+T_{1}\right) \geq \mathbb{E}\left(\mathbf{1}_{\mathcal{T}^{n}>T_{1}} \mathbb{P}_{Z_{T_{1}}^{n}}\left(\mathcal{T}^{n} \leq T\right)\right) \geq \mathbb{P}\left(d_{\mathbb{D}, T_{1}}\left(Z^{n}, \xi\right) \leq \delta_{3}\right) \mathrm{e}^{-n\left(\mathcal{I}_{\tilde{\xi}}^{T}(z)+2 \varepsilon\right)} \geq \mathrm{e}^{-n\left(\mathcal{I}_{\tilde{\xi}}^{T}(z)+3 \varepsilon\right)},
$$

where the lower bound for $\mathbb{P}\left(d_{\mathbb{D}, T_{1}}\left(Z^{n}, \xi\right) \leq \delta_{3}\right)$ is obtained by Proposition 5.1 and Lemma 5.9.

Step 3. We now establish (5.15). Let $p:=1-\mathrm{e}^{-n\left(\mathcal{I}_{\tilde{\xi}}^{T}(z)+3 \varepsilon\right)}$ and assume that for $k \in \mathbb{N}^{*}$, we have shown that for $\xi_{0} \in B_{\mathcal{W}_{1}}(\widehat{\xi}, \gamma)$ :

$$
\mathbb{P}\left(\mathcal{T}^{n}>k\left(T+T_{1}\right)\right) \leq p^{k}
$$

By the strong Markov property and since on $\left\{\mathcal{T}^{n}>k\left(T+T_{1}\right)\right\}, Z_{k\left(T+T_{1}\right)}^{n} \in B_{\mathcal{W}_{1}}(\widehat{\xi}, \gamma)$ converges to $\xi_{k\left(T+T_{1}\right)} \in$ $B_{\mathcal{W}_{1}}(\widehat{\xi}, \gamma)$ :

$$
\mathbb{P}\left(\mathcal{T}^{n}>(k+1)\left(T+T_{1}\right)\right) \leq \mathbb{E}\left(\mathbb{P}_{Z_{k\left(T+T_{1}\right)}^{n}}\left(\mathcal{T}^{n}>T+T_{1}\right) \mathbf{1}_{\mathcal{T}^{n}>k\left(T+T_{1}\right)}\right) \leq p \mathbb{P}\left(\mathcal{T}^{n} \geq k\left(T+T_{1}\right)\right) \leq p^{k+1} .
$$

By recursion, we obtain (5.20) for every $k \in \mathbb{N}^{*}$.

Step 4. We deduce for $\xi_{0} \in B_{\mathcal{W}_{1}}(\widehat{\xi}, \gamma)$ :

$$
\mathbb{E}\left(\frac{\mathcal{T}^{n}}{T+T_{1}}\right)=\sum_{k=0}^{+\infty} \int_{k}^{k+1} \mathbb{P}\left(\frac{\mathcal{T}^{n}}{T+T_{1}}>t\right) \mathrm{d} t \leq \sum_{k=0}^{+\infty} \mathbb{P}\left(\mathcal{T}^{n}>k\left(T+T_{1}\right)\right) \leq \frac{1}{1-p}=\mathrm{e}^{n\left(\mathcal{I}_{\tilde{\xi}}^{T}(z)+3 \varepsilon\right)},
$$


by definition of $p$. Then $\lim \sup _{n \rightarrow+\infty} \frac{1}{n} \log \mathbb{E}\left(\mathcal{T}^{n}\right) \leq \mathcal{I}_{\widehat{\xi}}^{T}(z)+3 \varepsilon$. The choice of $\varepsilon>0$ is arbitrary and the proposition is proved.

Remark 5.10. In order to obtain the inequalities (5.19), the fact that we only have a local minoration in (4.10) is not restrictive, since we consider deviations with respect to the path $\xi$, which belongs to $\mathfrak{G}(4.9)$.

Proof of Lemma 5.8. Let $\varepsilon>0, \delta \in] 0,1]$ and $\mu_{0} \in B_{T V}\left(\xi_{0}, \delta\right)$. We have $\sup _{t \in[0, T]}\left\langle z_{t}+z_{t}^{\mu_{0}}, 1\right\rangle \leq\left(2\left\langle\xi_{0}, 1\right\rangle+\right.$ 1) $\mathrm{e}^{\bar{b} T}=: A_{T}$ independent of $\mu_{0}$ and $\delta$.

Let $\phi \in \mathcal{C}_{b}^{1}\left(\mathbb{R}_{+}, \mathbb{R}_{+}\right)$such that $\|\phi\|_{\infty} \leq 1$. Let $t \in[0, T]$ and $(f:(a, s) \mapsto \phi(a+t-s)) \in \mathcal{C}_{b}^{1}\left(\mathbb{R}_{+} \times \mathbb{R}_{+}, \mathbb{R}\right)$. With a computation similar to (3.1):

$$
\begin{aligned}
\left|\left\langle z_{t}-z_{t}^{\mu_{0}}, \phi\right\rangle\right| & \leq\left|\int_{\mathbb{R}_{+}} \phi(a+t) z_{0}(\mathrm{~d} a)-\int_{\mathbb{R}_{+}} \phi(a+t) \mu_{0}(\mathrm{~d} a)\right| \\
& +\left|\int_{0}^{t} \int_{E} \psi(f)\left(h^{z}+1\right) \mathrm{d} m_{s}^{z, T} \mathrm{~d} s-\int_{0}^{t} \int_{E} \psi(f)\left(h^{z}+1\right) \mathrm{d} m_{s}^{z^{\mu_{0}}, T} \mathrm{~d} s\right| \\
& \leq\left\|z_{0}-\mu_{0}\right\|_{T V}+\left(\left\|h^{z}\right\|_{\infty}+1\right)\left[\bar{b}+\bar{d}+2 \eta A_{T}\right] \int_{0}^{t}\left\|z_{s}-z_{s}^{\mu_{0}}\right\|_{T V} \mathrm{~d} s .
\end{aligned}
$$

Taking the supremum in $\phi \in \mathcal{C}_{b}^{1}\left(\mathbb{R}_{+}, \mathbb{R}_{+}\right)$bounded by 1 gives:

$$
\left\|z_{t}-z_{t}^{\mu_{0}}\right\|_{T V} \leq \delta \exp \left(\left(\left\|h^{z}\right\|_{\infty}+1\right)\left[\bar{b}+\bar{d}+2 \eta A_{T}\right] T\right),
$$

by Gronwall's lemma and by choice of $\mu_{0}$. Choosing $\delta=\min \left(1, \varepsilon \mathrm{e}^{-\left(\left\|h^{z}\right\|_{\infty}+1\right)\left[\bar{b}+\bar{d}+2 \eta A_{T}\right] T}\right)$, we have $\left.\left.\delta \in\right] 0,1\right]$ and can upper bound the right hand side of (5.24) by $\varepsilon$, which proves Point (i).

Now let us consider Point (ii). For $f^{z}$ defined in Proposition 4.12 and using (4.29):

$$
\left|\mathcal{I}_{\mu_{0}}^{T}\left(z^{\mu_{0}}\right)-\mathcal{I}_{z_{0}}^{T}(z)\right| \leq\left\|h^{z} \psi\left(f^{z}\right)-\rho\left(\psi\left(f^{z}\right)\right)\right\|_{\infty} T\left(\bar{b}+\bar{d}+2 \eta A_{T}\right) \sup _{t \in[0, T]}\left\|z_{t}-z_{t}^{\mu_{0}}\right\|_{T V}
$$

Thanks to Point (i), there exists $\delta>0$ such that for all $\mu_{0} \in B\left(z_{0}, \delta\right),(5.25)$ is upper bounded by $\varepsilon$.

\subsubsection{Lower bound of the exit time}

We now consider the probability $\mathbb{P}\left(\mathcal{T}^{n}>\mathrm{e}^{n(\underline{V}-\delta)}\right)$ for $\delta>0$. We rely on the large deviation upper bound (4.10). The skeleton of the proof looks like its counterpart in finite dimension (see [12], Sect. 5), but the proofs of all the technical lemmas have to be changed, when dealing with our measure-valued processes. Another difficulty is that $\underline{V}$ is possibly infinite.

Proposition 5.11. Let $\xi_{0} \in B_{\mathcal{W}_{1}}(\widehat{\xi}, \gamma)$. Under Assumptions 2, 5, 8 :

1. If $\underline{V}<+\infty$, then $\forall \delta>0, \lim _{n \rightarrow+\infty} \mathbb{P}\left(\mathcal{T}^{n}>\mathrm{e}^{n(\underline{V}-\delta)}\right)=1$.

2. If $\underline{V}=+\infty$, then $\forall V>0, \lim _{n \rightarrow+\infty} \mathbb{P}\left(\mathcal{T}^{n}>\mathrm{e}^{n V}\right)=1$.

Let $\rho \in] 0, \gamma / 2[$. For such $\rho$, we define the following stopping times:

$$
\sigma_{\rho}=\inf \left\{t \geq 0, Z_{t}^{n} \in B_{\mathcal{W}_{1}}(\widehat{\xi}, \rho) \cup B_{\mathcal{W}_{1}}^{c}(\widehat{\xi}, \gamma)\right\} .
$$

$\sigma_{\rho}$ is the first time when $\left(Z_{t}^{n}\right)_{t \in \mathbb{R}_{+}}$enters $B_{\mathcal{W}_{1}}(\widehat{\xi}, \rho)$ or leaves $B_{\mathcal{W}_{1}}(\widehat{\xi}, \gamma)$. The proof of Proposition 5.11 is based on the following lemmas, proved at the end of the section. The interesting case is when $\rho \leq \mathcal{W}_{1}\left(\xi_{0}, \widehat{\xi}\right), \xi_{0} \in$ $B_{\mathcal{W}_{1}}(\widehat{\xi}, \gamma) \backslash B_{\mathcal{W}_{1}}(\widehat{\xi}, \rho)$. The estimates of Lemmas 5.12, 5.13 and 5.14 are automatically satisfied if $\rho>\mathcal{W}_{1}\left(\xi_{0}, \widehat{\xi}\right)$.

Lemma 5.12. Let $\rho \in] 0, \gamma / 2\left[\lim _{t \rightarrow+\infty} \lim \sup _{n \rightarrow+\infty} \frac{1}{n} \log \mathbb{P}\left(\sigma_{\rho}>t\right)=-\infty\right.$. 
Lemma 5.13. (i) $\lim _{\rho \rightarrow 0} \lim \sup _{n \rightarrow+\infty} \frac{1}{n} \log \mathbb{P}\left(Z_{\sigma_{\rho}}^{n} \in B_{\mathcal{W}_{1}}^{c}(\widehat{\xi}, \gamma)\right) \leq-\underline{V}$ (where $\underline{V}$ is possibly equal to $+\infty$ ).

(ii) We deduce that for $\rho \in] 0, \gamma / 2\left[\lim _{n \rightarrow+\infty} \mathbb{P}\left(Z_{\sigma_{\rho}}^{n} \in B_{\mathcal{W}_{1}}(\widehat{\xi}, \rho)\right)=1\right.$.

Lemma 5.14. Let $\rho \in] 0, \gamma / 2[. \forall c>0, \exists T(c, \rho)>0$,

$$
\limsup _{n \rightarrow+\infty} \frac{1}{n} \log \mathbb{P}\left(\sup _{t \in[0, T(c, \rho)]} \mathcal{W}_{1}\left(Z_{t}^{n}, Z_{0}^{n}\right) \geq \rho\right)<-c .
$$

Lemmas $5.12,5.13$ and 5.14 tell us that the following probabilities are exponentially small:

1. the probability that the path $\left(Z_{s}^{n}\right)_{s \in[0, t]}$ remains in the set $B_{\mathcal{W}_{1}}(\widehat{\xi}, \gamma) \backslash B_{\mathcal{W}_{1}}(\widehat{\xi}, \rho)$ when $t \rightarrow+\infty$,

2. the probability that $Z^{n}$ leaves this set by leaving $B_{\mathcal{W}_{1}}(\widehat{\xi}, \gamma)$,

3. the probability that $Z^{n}$ "strongly" deviates from its initial condition $Z_{0}^{n}$ in short time.

Notice that the use of $\mathcal{W}_{1}$ is fundamental in the proof of Lemma 5.14.

Proof of Proposition 5.11. Let $\delta>0$ and let:

$$
\widetilde{V}=\left\{\begin{array}{cl}
\underline{V} & \text { if } \underline{V}<+\infty \\
V+\delta & \text { if } \underline{V}=+\infty
\end{array}\right.
$$

with an arbitrary $V>0$ as in the terms of the Proposition. By Lemma 5.13,

$$
\exists \rho \in] 0, \gamma / 2\left[\limsup _{n \rightarrow+\infty} \frac{1}{n} \log \mathbb{P}\left(Z_{\sigma_{\rho}}^{n} \in B_{\mathcal{W}_{1}}^{c}(\widehat{\xi}, \gamma)\right)<-\widetilde{V}+\delta / 4\right.
$$

and: $\exists n_{0} \in \mathbb{N}^{*}, \forall n \geq n_{0}$,

$$
\mathbb{P}\left(Z_{\sigma_{\rho}}^{n} \in B_{\mathcal{W}_{1}}^{c}(\widehat{\xi}, \gamma)\right)<\mathrm{e}^{-n(\widetilde{V}-\delta / 2)}
$$

We define the following stopping times: $\theta_{0}=0$ and $\forall k \in \mathbb{N}$,

$$
\begin{aligned}
\tau_{k} & =\inf \left\{t \geq \theta_{k}, \quad Z_{t}^{n} \in B_{\mathcal{W}_{1}}(\widehat{\xi}, \rho) \cup B_{\mathcal{W}_{1}}^{c}(\widehat{\xi}, \gamma)\right\}, \\
\theta_{k+1} & =\inf \left\{t \geq \tau_{k}, \quad Z_{t}^{n} \in B_{\mathcal{W}_{1}}^{c}(\widehat{\xi}, 2 \rho)\right\},
\end{aligned}
$$

with the convention $\theta_{k+1}=+\infty$ if $Z_{\tau_{k}}^{n} \in B_{\mathcal{W}_{1}}^{c}(\widehat{\xi}, \gamma) \cdot \tau_{k}$ is the first time of exit from $B_{\mathcal{W}_{1}}(\widehat{\xi}, \gamma) \backslash B_{\mathcal{W}_{1}}(\widehat{\xi}, \rho)$ after $\theta_{k}$, and if $Z_{\tau_{k}}^{n} \in B_{\mathcal{W}_{1}}(\widehat{\xi}, \rho), \theta_{k+1}$ is the first time of exit of $B_{\mathcal{W}_{1}}(\widehat{\xi}, 2 \rho)$. Notice that the exit time $\mathcal{T}^{n}$ is one of the $\tau_{k}, k \in \mathbb{N}$.

Let $q \in \mathbb{N}^{*}$ and $T_{0}=T(\widetilde{V}, \rho)$ as given by Lemma 5.14. The event $\left\{\mathcal{T}^{n} \leq q T_{0}\right\}$ implies that there exists $k \in \llbracket 0, q \rrbracket$ such that either $\mathcal{T}^{n}=\tau_{k}$ or one of the $q$ first "excursions" $\left[\tau_{k-1}, \tau_{k}\right]$ for $k \in \llbracket 1, q \rrbracket$ is of length upper bounded by $T_{0}$. In this last case, one of the trajectories $\left[\tau_{k-1}, \theta_{k}\left[\right.\right.$ is covered in less than $T_{0}$. Thus:

$$
\mathbb{P}\left(\mathcal{T}^{n} \leq q T_{0}\right) \leq \sum_{k=1}^{q}\left[\mathbb{P}\left(\mathcal{T}^{n}=\tau_{k}\right)+\mathbb{P}\left(\min _{1 \leq k \leq q}\left(\theta_{k}-\tau_{k-1}\right) \leq T_{0}\right)\right]+\mathbb{P}\left(\mathcal{T}^{n}=\tau_{0}\right) .
$$

By Markov strong property and by (5.29), $\exists n_{0}>0, \forall n \geq n_{0}, \forall k \in \mathbb{N}^{*}$,

$$
\mathbb{P}\left(\mathcal{T}^{n}=\tau_{k}\right)=\mathbb{P}\left(\mathbb{P}_{Z_{\theta_{k}}^{n}}\left(\mathcal{T}^{n}=\tau_{0}\right) \mathbf{1}_{\mathcal{T}^{n}>\tau_{k-1}}\right)=\mathbb{P}\left(\mathbb{P}_{Z_{\theta_{k}}^{n}}\left(Z_{\sigma_{\rho}}^{n} \in B_{\mathcal{W}_{1}}^{c}(\widehat{\xi}, \gamma)\right) \mathbf{1}_{\mathcal{T}^{n}>\tau_{k-1}}\right) \leq \mathrm{e}^{-n(\widetilde{V}-\delta / 2)},
$$

by noticing that for sufficiently large $n, Z_{\theta_{k}}^{n}$ belongs to $B_{\mathcal{W}_{1}}(\widehat{\xi}, \gamma)$. 
By choice of $T_{0}$, by the strong Markov property: $\forall n \geq n_{0}, \forall k \in \mathbb{N}^{*}$,

$$
\mathbb{P}\left(\theta_{k}-\tau_{k-1} \leq T_{0}\right) \leq \mathbb{P}\left(\sup _{t \in\left[0, T_{0}\right]} \mathcal{W}_{1}\left(Z_{t}^{n}, Z_{0}^{n}\right) \geq \rho\right) \leq \mathrm{e}^{-n(\widetilde{V}-\delta / 2)} .
$$

By (5.32), (5.33), (5.34) : $\forall q \in \mathbb{N}^{*}$,

$$
\mathbb{P}\left(\mathcal{T}^{n} \leq q T_{0}\right) \leq \mathbb{P}\left(\mathcal{T}^{n}=\tau_{0}\right)+2 q \mathrm{e}^{-n(\tilde{V}-\delta / 2)} .
$$

Since $\left\{\mathcal{T}^{n}=\tau_{0}\right\}=\left\{Z_{\sigma_{\rho}}^{n} \in B_{\mathcal{W}_{1}}^{c}(\widehat{\xi}, \gamma)\right\}$, then, for the choice of $q=\left[\mathrm{e}^{n(\widetilde{V}-\delta)} / T_{0}\right]+1$ :

$$
\mathbb{P}\left(\mathcal{T}^{n} \leq \mathrm{e}^{n(\tilde{V}-\delta)}\right) \leq \mathbb{P}\left(\mathcal{T}^{n} \leq q T_{0}\right) \leq \mathbb{P}\left(Z_{\sigma_{\rho}}^{n} \in B_{\mathcal{W}_{1}}^{c}(\widehat{\xi}, \gamma)\right)+\frac{4}{T_{0}} \mathrm{e}^{-n \delta / 2}
$$

The right hand side of (5.36) tends to 0 when $n \rightarrow+\infty$, as $\lim \mathbb{P}\left(Z_{\sigma_{\rho}}^{n} \in B_{\mathcal{W}_{1}}^{c}(\widehat{\xi}, \gamma)\right)=0$, by Lemma 5.13.

Proof of Lemma 5.12. If $\xi_{0} \in B_{\mathcal{W}_{1}}(\widehat{\xi}, \rho)$, Lemma 5.12 is true. Assume that $\rho \leq \mathcal{W}_{1}\left(\widehat{\xi}, \xi_{0}\right)$. Then, $\xi_{0} \in$ $B_{\mathcal{W}_{1}}(\widehat{\xi}, \gamma) \backslash B_{\mathcal{W}_{1}}(\widehat{\xi}, \rho)$. Let $L>0$. It is sufficient to show that: $\exists T=T(L)>0$,

$$
\limsup _{n \rightarrow+\infty} \frac{1}{n} \log \mathbb{P}\left(\sigma_{\rho}>T\right)<-L \text {. }
$$

By Point (iii) of Proposition 5.4: $\forall \xi_{0} \in B_{\mathcal{W}_{1}}(\widehat{\xi}, \gamma), \exists T_{1}(\rho, \widehat{\xi}, \gamma)>0, \forall t \geq T_{1}, \mathcal{W}_{1}\left(\xi_{t}^{\xi_{0}}, \widehat{\xi}\right)<\rho / 2$.

Let $T \geq T_{1}$. If the path $\left(Z_{t}^{n}\right)_{t \in[0, T]}$ remains in $B_{\mathcal{W}_{1}}(\widehat{\xi}, \gamma) \backslash B_{\mathcal{W}_{1}}(\widehat{\xi}, \rho)$, we have necessarily $\mathcal{W}_{1}\left(Z_{T}^{n}, \xi_{T}^{\xi_{0}}\right)>\rho / 2$. As $\left(\mathcal{L}\left(Z^{n}\right)\right)_{n \in \mathbb{N}^{*}}$ is exponentially tight, $\exists K(L, T) \subset \mathbb{D}_{T}$ compact, $\exists n_{0} \in \mathbb{N}^{*}, \forall n \geq n_{0}$,

$$
\mathbb{P}\left(Z^{n} \notin K(L, T)\right) \leq \mathrm{e}^{-n L} .
$$

The following set $\mathcal{A}(T)=\operatorname{adh}\left\{z \in \mathbb{D}_{T}, \forall t \in[0, T], z_{t} \in B_{\mathcal{W}_{1}}(\widehat{\xi}, \gamma) \backslash B_{\mathcal{W}_{1}}(\widehat{\xi}, \rho)\right\} \cap K(L, T)$ is compact, does not contain $\xi$ by choice of $T$ and $\forall z \in \mathcal{A}(T), \mathcal{I}_{\xi_{0}}^{T}(z)>0$ (Lem. 5.9). Then:

$$
\begin{aligned}
\limsup _{n \rightarrow+\infty} \frac{1}{n} \log \mathbb{P}\left(\sigma_{\rho}>T\right) & \leq \max \left(\limsup _{n \rightarrow+\infty} \frac{1}{n} \log \mathbb{P}\left(Z^{n} \in \mathcal{A}(T)\right), \limsup _{n \rightarrow+\infty} \frac{1}{n} \log \mathbb{P}\left(Z^{n} \notin K(L, T)\right)\right) \\
& \leq \max \left(-\inf _{z \in \mathcal{A}(T)} \mathcal{I}_{\xi_{0}}^{T}(z),-L\right)<0 .
\end{aligned}
$$

It remains to show that for sufficiently large $T$,

$$
\inf _{z \in \mathcal{A}(T)} \mathcal{I}_{\xi_{0}}^{T}(z)>L
$$

Let $z \in \mathcal{A}(T)$ starting from $\xi_{0} \in B_{\mathcal{W}_{1}}(\widehat{\xi}, \gamma) \backslash B_{\mathcal{W}_{1}}(\widehat{\xi}, \rho)$ with $T \geq T_{1}$. Let $\phi \in \mathcal{C}^{1}\left(\mathbb{R}_{+}, \mathbb{R}\right)$ be bounded by 1 , $t \in[0, T]$ and $f:(a, s) \in \mathbb{R}_{+}^{2} \mapsto f_{s}(a)=\phi(a+t-s) \in \mathbb{R}$. We have:

$$
\begin{aligned}
\left\langle z_{t}-\xi_{t}, \phi\right\rangle= & \int_{0}^{t} \int_{\mathbb{R}_{+}}\left(b(a) \phi(t-s)-\phi(a+t-s)\left(d(a)-\eta\left\langle z_{s}, 1\right\rangle\right)\right)\left(z_{s}-\xi_{s}\right)(d a) \mathrm{d} s \\
& -\int_{0}^{t} \int_{\mathbb{R}_{+}} \phi(a+t-s) \eta\left(\left\langle z_{s}-\xi_{s}, 1\right\rangle\right) \xi_{s}(d a) \mathrm{d} s+\int_{0}^{t} \int_{E} \psi(f) h^{z} \mathrm{~d} m_{s}^{z, T} \mathrm{~d} s .
\end{aligned}
$$


For $z \in \mathcal{A}(T)$ and by choice of $\gamma, \sup _{t \in[0, T]}\left(\left\langle\xi_{t}, 1\right\rangle+\left\langle z_{t}, 1\right\rangle\right) \leq 4\langle\widehat{\xi}, 1\rangle \mathrm{e}^{\bar{b} T}:=A_{T}$, which is finite and does not depend on $\xi_{0} \in B_{\mathcal{W}_{1}}(\widehat{\xi}, \gamma)$ nor on $z \in \mathcal{A}(T)$. We then deduce that:

$$
\left|\left\langle z_{t}-\xi_{t}, \phi\right\rangle\right| \leq\left(\bar{b}+\bar{d}+2 \eta A_{T}\right) \int_{0}^{t} \sup _{u \in[0, s]}\left\|z_{u}-\xi_{u}\right\|_{T V} \mathrm{~d} s+\int_{0}^{t} \int_{E}\left|h^{z}\right| \mathrm{d} m_{s}^{z, T} \mathrm{~d} s .
$$

Taking the supremum in $\phi$ in the left hand side, by Gronwall's inequality and by (5.1):

$$
\sup _{t \in[0, T]} \mathcal{W}_{1}\left(z_{t}, \xi_{t}\right) \leq \sup _{t \in[0, T]}\left\|z_{t}-\xi_{t}\right\|_{T V} \leq\left(\int_{0}^{T} \int_{E}\left|h^{z}\right| \mathrm{d} m_{s}^{z, T} \mathrm{~d} s\right) \mathrm{e}^{\left(\bar{b}+\bar{d}+2 \eta A_{T}\right) T} .
$$

Since $\sup _{t \in[0, T]} \mathcal{W}_{1}\left(z_{t}, \xi_{t}\right) \geq \rho / 2$ :

$$
\int_{0}^{T} \int_{E}\left|h^{z}\right| \frac{\mathrm{d} m_{s}^{z, T} \mathrm{~d} s}{\int_{0}^{T} \int_{E} \mathrm{~d} m_{s}^{z, T} \mathrm{~d} s} \geq \frac{\rho}{2} \frac{1}{\int_{0}^{T} \int_{E} \mathrm{~d} m_{s}^{z, T} \mathrm{~d} s} \mathrm{e}^{-\left(\bar{b}+\bar{d}+2 \eta A_{T}\right) T}
$$

and since $\rho^{*}$ is an increasing function on $\mathbb{R}_{+}$:

$$
\rho^{*}\left(\int_{0}^{T} \int_{E}\left|h^{z}\right| \frac{\mathrm{d} m_{s}^{z, T} \mathrm{~d} s}{\int_{0}^{T} \int_{E} \mathrm{~d} m_{s}^{z, T} \mathrm{~d} s}\right) \geq \rho^{*}\left(\frac{\rho}{2} \frac{1}{\left(\bar{b}+\bar{d}+\eta A_{T}\right) A_{T} T} \mathrm{e}^{-\left(\bar{b}+\bar{d}+2 \eta A_{T}\right) T}\right) .
$$

On the other hand, by Jensen's inequality:

$$
\rho^{*}\left(\int_{0}^{T} \int_{E}\left|h^{z}\right| \frac{\mathrm{d} m_{s}^{z, T} \mathrm{~d} s}{\int_{0}^{T} \int_{E} \mathrm{~d} m_{s}^{z, T} \mathrm{~d} s}\right) \leq \int_{0}^{T} \int_{E} \rho^{*}\left(\left|h^{z}\right|\right) \frac{\mathrm{d} m_{s}^{z, T} \mathrm{~d} s}{\int_{0}^{T} \int_{E} \mathrm{~d} m_{s}^{z, T} \mathrm{~d} s}=\frac{\mathcal{I}_{\xi_{0}}^{T}(z)}{\int_{0}^{T} \int_{E} \mathrm{~d} m_{s}^{z, T} \mathrm{~d} s} .
$$

By (5.39) and (5.40), and for $T=T_{1}$ :

$$
\begin{aligned}
\int_{0}^{T_{1}} \int_{E} \rho^{*}\left(h^{z}\right) \mathrm{d} m_{s}^{z, T_{1}} \mathrm{~d} s & =\mathcal{I}_{\xi_{0}}^{T_{1}}(z) \geq\left(\int_{0}^{T_{1}} \int_{E} \mathrm{~d} m_{s}^{z, T_{1}} \mathrm{~d} s\right) \rho^{*}\left(\frac{\rho}{2} \frac{1}{\left(\bar{b}+\bar{d}+\eta A_{T_{1}}\right) A_{T_{1}} T_{1}} \mathrm{e}^{-\left(\bar{b}+\bar{d}+2 \eta A_{T_{1}}\right) T_{1}}\right) \\
& \geq\left(\inf _{z \in \mathcal{A}\left(T_{1}\right)} \int_{0}^{T_{1}} \int_{E} \mathrm{~d} m_{s}^{z, T_{1}} \mathrm{~d} s\right) \rho^{*}\left(\frac{\rho}{2} \frac{\mathrm{e}^{-\left(\bar{b}+\bar{d}+2 \eta A_{T_{1}}\right) T_{1}}}{\left(\bar{b}+\bar{d}+\eta A_{T_{1}}\right) A_{T_{1}} T_{1}}\right):=C_{2}>0,
\end{aligned}
$$

by compactness of $\mathcal{A}\left(T_{1}\right)$ with paths that do not vanish (by choice of $\gamma$ and since they remain in $\left.\operatorname{adh}\left(B_{\mathcal{W}_{1}}(\widehat{\xi}, \gamma)\right)\right)$. Notice that the constant $C_{2}$ does not depend on the particular choice of the initial condition $\xi_{0} \in B_{\mathcal{W}_{1}}(\widehat{\xi}, \gamma) \backslash$ $B_{\mathcal{W}_{1}}(\widehat{\xi}, \rho)$ nor on the path $z \in \mathcal{A}(T)$.

Let now $T>0$ be such that $T>J T_{1}$ with $J \in \mathbb{N}^{*}$. We have:

$$
\mathcal{I}_{\xi_{0}}^{T}(z) \geq \sum_{j=1}^{J} \int_{(j-1) T_{1}}^{j T_{1}} \int_{E} \rho^{*}\left(h^{z}\right) \mathrm{d} m_{s}^{z, T} \mathrm{~d} s=\sum_{j=1}^{J} \int_{0}^{T_{1}} \int_{E} \rho^{*}\left(h^{z^{j}}\right) \mathrm{d} m_{s}^{z^{j}, T} \mathrm{~d} s,
$$

where $\forall t \in\left[0, T_{1}\right], z_{t}^{j}=z_{(j-1) T_{1}+t}$ is a path in $\mathcal{A}\left(T_{1}\right)$ which solves: $\forall\left(f:(a, s) \mapsto f_{s}(a)\right) \in \mathcal{C}_{b}^{1}\left(\mathbb{R}_{+} \times\left[0, T_{1}\right], \mathbb{R}\right)$,

$$
\left\langle z_{t}^{j}, f_{t}\right\rangle=\left\langle z_{(j-1) T_{1}}, f_{0}\right\rangle+\int_{0}^{t}\left\langle z_{s}^{j}, \frac{\partial f_{s}}{\partial a}+\frac{\partial f_{s}}{\partial s}\right\rangle \mathrm{d} s+\int_{0}^{t} \int_{E}\left(1+h^{z^{j}}\right) \psi(f) \mathrm{d} m_{s}^{z^{j}, T} \mathrm{~d} s
$$

which is an equation obtained by the perturbation of $(1.2)$ with $h^{z^{j}}(a, u, s)=h^{z}\left(a, u,(j-1) T_{1}+s\right)$ for $s \in\left[0, T_{1}\right]$. By (5.41) and by recursion $\mathcal{I}_{\xi_{0}}^{T}(z) \geq J C_{2}$. This proves (5.37). 
Proof of Lemma 5.13. Consider Point (i). Let $\rho \in] 0, \gamma / 2[$ and $R>0$ such that $\underline{V}(R)<+\infty$. By Lemma 5.12:

$$
\exists T_{2}>0, \exists n_{0} \in \mathbb{N}^{*}, \forall n \geq n_{0}, \mathbb{P}\left(\sigma_{\rho}>T_{2}\right)<\mathrm{e}^{-n \underline{\underline{V}}(R)},
$$

where $\underline{V}(R)$ is defined in (5.9). For $T>0$, we define:

$$
A(T):=\left\{z \in \mathbb{D}\left([0, T], \mathcal{M}_{F}\left(\mathbb{R}_{+}\right)\right) \mid \exists t \in[0, T], z_{t} \notin B_{\mathcal{W}_{1}}(\widehat{\xi}, \gamma)\right\}
$$

which is closed since $z \mapsto \sup _{t \in[0, T]} \mathcal{W}_{1}(z, \widehat{\xi})$ is continuous. From (5.9): $\forall \xi_{0} \in B_{\mathcal{W}_{1}}(\widehat{\xi}, 2 \rho), \inf _{z \in A\left(T_{2}\right)} \mathcal{I}_{\xi_{0}}^{T_{2}}(z) \geq$ $\underline{V}(2 \rho)$. Using the large deviation upper bound (Th. 4.3):

$$
\limsup _{n \rightarrow+\infty} \frac{1}{n} \log \mathbb{P}_{Z_{0}^{n} \in B_{\mathcal{W}_{1}}(\widehat{\xi}, \gamma) \backslash B_{\mathcal{W}_{1}}(\widehat{\xi}, \rho)}\left(Z^{n} \in A\left(T_{2}\right)\right) \leq-\inf _{z \in A\left(T_{2}\right)} \mathcal{I}_{\xi_{0}}^{T_{2}}(z) \leq-\underline{V}(2 \rho) .
$$

By (5.43) and (5.45) :

$$
\begin{aligned}
\limsup _{n \rightarrow+\infty} \frac{1}{n} \log \mathbb{P}\left(Z_{\sigma_{\rho}}^{n} \in B_{\mathcal{W}_{1}}^{c}(\widehat{\xi}, \gamma)\right) \leq \\
\quad \max \left(\limsup _{n \rightarrow+\infty} \frac{1}{n} \log \mathbb{P}\left(\sigma_{\rho}>T_{2}\right), \limsup _{n \rightarrow+\infty} \frac{1}{n} \log \mathbb{P}\left(Z^{n} \in A\left(T_{2}\right)\right)\right) \leq \max (-\underline{V}(R),-\underline{V}(2 \rho)) .
\end{aligned}
$$

By (5.10), $\lim _{\rho \rightarrow 0} \lim \sup _{n \rightarrow+\infty} \frac{1}{n} \log \mathbb{P}\left(Z_{\sigma_{\rho}}^{n} \in B_{\mathcal{W}_{1}}^{c}(\widehat{\xi}, \gamma)\right) \leq-\underline{V}(R)$, and letting $R$ tend to 0 , we obtain the result of Point (i), since the left hand side does not depend on $R$.

Let us consider Point (ii). Let $\varepsilon>0, \rho \in] 0, \gamma / 2[$ and $R>0$ satisfying $\underline{V}(R)<+\infty$ and:

$$
\limsup _{n \rightarrow+\infty} \frac{1}{n} \log \mathbb{P}\left(Z_{\sigma_{\rho}}^{n} \in B_{\mathcal{W}_{1}}^{c}(\widehat{\xi}, \gamma)\right) \leq-\underline{V}(R)+\frac{\varepsilon}{2},
$$

(the existence of such $R$ is given by (i)). Then $\exists n_{0} \in \mathbb{N}^{*}, \forall n \geq n_{0}, \mathbb{P}\left(Z_{\sigma_{\rho}}^{n} \in B_{\mathcal{W}_{1}}^{c}(\widehat{\xi}, \gamma)\right) \leq \mathrm{e}^{-n \underline{V}(R)+n \varepsilon}$, and:

$$
\mathbb{P}\left(Z_{\sigma_{\rho}}^{n} \in B_{\mathcal{W}_{1}}(\widehat{\xi}, \rho)\right)=1-\mathbb{P}\left(Z_{\sigma_{\rho}}^{n} \in B_{\mathcal{W}_{1}}^{c}(\widehat{\xi}, \gamma)\right) \geq 1-\mathrm{e}^{-n(\underline{\underline{V}}(R)-\varepsilon)} .
$$

Choosing $\varepsilon<\underline{V}(R)$ and letting $n$ tend to $+\infty$ completes the proof.

Proof of Lemma 5.14. Let $T>0$ and $c>0$. By Lemma 4.6 (i), there exists $N=N(c, T)>0$ such that:

$$
\limsup _{n \rightarrow+\infty} \frac{1}{n} \log \mathbb{P}\left(\zeta_{N}^{n}<T\right)<-c .
$$

Let $\phi \in \mathcal{C}_{b}^{1}\left(\mathbb{R}_{+}, \mathbb{R}\right) 1$-Lipschitz continuous bounded by $1, t \in[0, T]$, and $f(a, s)=\phi(a+t-s) \in \mathcal{C}_{b}^{1}\left(\mathbb{R}_{+} \times \mathbb{R}_{+}, \mathbb{R}\right)$. With the notation of Definition 2.4:

$$
\begin{aligned}
\left|\left\langle Z_{t \wedge \zeta_{N}^{n}}^{n}, \phi\right\rangle-\left\langle Z_{0}^{n}, \phi\right\rangle\right| \leq\left|\int_{\mathbb{R}_{+}}(\phi(a+t)-\phi(a)) Z_{0}^{n}(d a)\right|+\mid \frac{1}{n} \int_{0}^{t \wedge \zeta_{N}^{n}} \int_{\mathcal{E}} \mathbf{1}_{\left\{i \leq N_{s}^{n}\right\}}\left[f(0, s) \mathbf{1}_{0 \leq m_{1}\left(s, Z_{s_{-}}^{n}, i\right)}\right. \\
\left.-f\left(\pi_{i}\left(Z_{s_{-}}^{n}\right), s\right) \mathbf{1}_{m_{1}\left(s, Z_{s_{-}}^{n}, i\right) \leq \theta<m_{2}\left(s, Z_{s_{-}}^{n}, i\right)}\right] Q(d s, d i, d \theta) \mid \leq t\left\langle Z_{0}^{n}, 1\right\rangle+\frac{\mathcal{N}(t)}{n},
\end{aligned}
$$


where $\mathcal{N}(t)$ is a Poisson random variable with parameter $t N(\bar{b}+\bar{d}+\eta N)$. We deduce:

$$
\begin{gathered}
\sup _{s \in[0, t]} \mathcal{W}_{1}\left(Z_{s \wedge \zeta_{N}^{n}}^{n}, Z_{0}^{n}\right) \leq t\left\langle Z_{0}^{n}, 1\right\rangle+\frac{\mathcal{N}(t)}{n} \\
\text { and: } \mathbb{P}\left(\sup _{s \in[0, t]} \mathcal{W}_{1}\left(Z_{s \wedge \zeta_{N}^{n}}^{n}, Z_{0}^{n}\right) \geq \rho\right) \leq \mathbb{P}\left(t\left\langle Z_{0}^{n}, 1\right\rangle \geq \frac{\rho}{2}\right)+\mathbb{P}\left(\frac{\mathcal{N}(t)}{n} \geq \frac{\rho}{2}\right) .
\end{gathered}
$$

Since:

$$
\limsup _{n \rightarrow+\infty} \frac{1}{n} \log \mathbb{P}\left(\mathcal{N}(t) \geq \frac{n \rho}{2}\right) \leq \frac{\rho}{2} \log (t N(\bar{b}+\bar{d}+\eta N))
$$

which tends to $-\infty$ when $t \rightarrow 0$. Then: $\left.\exists T_{3} \in\right] 0, T\left[, \forall t \in\left[0, T_{3}\right]\right.$,

$$
\limsup _{n \rightarrow+\infty} \frac{1}{n} \log \mathbb{P}\left(\mathcal{N}(t) \geq \frac{n \rho}{2}\right)<-c .
$$

By Point 2 of Assumptions $5, \exists n_{0} \in \mathbb{N}^{*}, \forall n \geq n_{0},\left\langle Z_{0}^{n}, 1\right\rangle<C_{0}+\left\langle\xi_{0}, 1\right\rangle$. Then, for $T_{4}<\rho /\left(2\left(C_{0}+\left\langle\xi_{0}, 1\right\rangle\right)\right)$ : $\forall t \in\left[0, T_{4}\right], \forall n \geq n_{0}, \mathbb{P}\left(t\left\langle Z_{0}^{n}, 1\right\rangle>\frac{\rho}{2}\right)=0$.

Hence, for $t \in\left[0, T_{3} \wedge T_{4} \wedge T\right]$ :

$$
\begin{aligned}
\limsup _{n \rightarrow+\infty} \frac{1}{n} \log \mathbb{P}\left(\sup _{s \in[0, t]} \mathcal{W}_{1}\left(Z_{t}^{n}, Z_{0}^{n}\right) \geq \rho\right) \leq \max \left[\limsup _{n \rightarrow+\infty} \frac{1}{n} \log \mathbb{P}\left(\zeta_{N}^{n}<T\right),\right. \\
\left.\limsup _{n \rightarrow+\infty} \frac{1}{n} \log \mathbb{P}\left(t\left\langle Z_{0}^{n}, 1\right\rangle \geq \frac{\rho}{2}\right), \limsup _{n \rightarrow+\infty} \frac{1}{n} \log \mathbb{P}\left(\mathcal{N}(t) \geq \frac{n \rho}{2}\right)\right]<-c .
\end{aligned}
$$

This concludes the proof of Proposition 5.11.

Acknowledgements. I am greatly indebted to Sylvie Méléard for having proposed this subject to me and for her constant support during this work. I also wish to thank Christian Léonard for numerous helpful discussions and pertinent corrections.

\section{REFERENCES}

[1] D. Aldous, Stopping times and tightness. Ann. Probab. 6 (1978) 335-340.

[2] K.B. Athreya and P.E. Ney, Branching Processes. Springer edition (1970).

[3] R. Bellman and T.E. Harris, On age-dependent binary branching processes. Ann. Math. 55 (1952) 280-295.

[4] P. Billingsley, Convergence of Probability Measures. John Wiley \& Sons (1968).

[5] E. Bishop and R.R. Phelps, The support functionals of a convex set, in Proc. Sympos. Pure Math. Amer. Math. Soc., Ed. Providence 7 (1963) 27-35.

[6] S. Busenberg and M. Iannelli, A class of nonlinear diffusion problems in age-dependent population dynamics. Nonlinear Anal. 7 (1983) 501-529.

[7] N. Champagnat, R. Ferrière and S. Méléard, Individual-based probabilistic models of adpatative evolution and various scaling approximations, in Proceedings of the 5th seminar on Stochastic Analysis, Random Fields and Applications, Probability in Progress Series, Ascona, Suisse (2006). Birkhauser.

[8] N. Champagnat, R. Ferrière and S. Méléard, Unifying evolutionary dynamics: from individual stochastic processes to macroscopic models via timescale separation. Theoretical Population Biology (2006).

[9] K.S. Crump and C.J. Mode, A general age-dependent branching process i. J. Math. Anal. Appl. 24 (1968) $494-508$.

[10] K.S. Crump and C.J. Mode, A general age-dependent branching process ii. J. Math. Anal. Appl. 25 (1969) 8-17.

[11] D.A. Dawson and J. Gärtner, Large deviations from the McKean-Vlasov limit for weakly interacting diffusions. Stochastics 20 (1987) 247-308.

[12] A. Dembo and O. Zeitouni, Large Deviation Techniques and Applications. Jones and Bartlett Publishers, Boston (1993). 
[13] R.A. Doney, Age-dependent birth and death processes. Z. Wahrscheinlichkeitstheorie verw. 22 (1972) 69-90.

[14] I. Ekeland and R. Temam, Convex Analysis and Variational Problems. North-Holland, Amsterdam (1976).

[15] L.C. Evans, Partial Differential Equations, Grad. Stud. Math. 19 American Mathematical Society (1998).

[16] H. Von Foerster, Some remarks on changing populations, in The Kinetics of Cellular Proliferation, Grune \& Stratton Ed., New York (1959) 382-407.

[17] N. Fournier and S. Méléard, A microscopic probabilistic description of a locally regulated population and macroscopic approximations. Ann. Appl. Probab. 14 (2004) 1880-1919.

[18] M.I. Freidlin and A. Ventzell, Random Perturbations of Dynamical Systems. Springer-Verlag (1984).

[19] F. Galton and H.W. Watson, On the probability of the extinction of families. J. Anthropol. Inst. Great B. and Ireland 4 (1874) $138-144$.

[20] C. Graham and S. Méléard, A large deviation principle for a large star-shaped loss network with links of capacity one. Markov Processes and Related Fields 3 (1997) 475-492.

[21] C. Graham and S. Méléard, An upper bound of large deviations for a generalized star-shaped loss network. Markov Processes and Related Fields 3 (1997) 199-224.

[22] M.E. Gurtin and R.C. MacCamy, Nonlinear age-dependent population dynamics. Arch. Rat. Mech. Anal. 54 (1974) $281-300$.

[23] T.E. Harris, The Theory of Branching Processes. Springer, Berlin (1963).

[24] R.B. Israel, Existence of phase transitions for long-range interactions. Comm. Math. Phys. 43 (1975) 59-68.

[25] J. Jacod and A.N. Shiryaev, Limit Theorems for Stochastic Processes. Springer-Verlag, Berlin (1987).

[26] P. Jagers, A general stochastic model for population development. Skand. Aktuarietidskr 52 (1969) 84-103.

[27] P. Jagers and F. Klebaner, Population-size-dependent and age-dependent branching processes. Stochastic Process Appl. 87 (2000) 235-254.

[28] A. Jakubowski, On the skorokhod topology. Ann. Inst. H. Poincaré 22 (1986) 263-285.

[29] A. Joffe and M. Métivier, Weak convergence of sequences of semimartingales with applications to multitype branching processes. Advances in Applied Probability 18 (1986) 20-65.

[30] D.G. Kendall, Stochastic processes and population growth. J. Roy. Statist. Sec., Ser. B 11 (1949) 230-264.

[31] C. Kipnis and C. Léonard, Grandes déviations pour un système hydrodynamique asymétrique de particules indépendantes. Ann. Inst. H. Poincaré 31 (1995) 223-248.

[32] C. Léonard, On large deviations for particle systems associated with spatially homogeneous boltzmann type equations. Probab. Theory Related Fields 101 (1995) 1-44.

[33] T.R. Malthus, An Essay on the Principle of Population. J. Johnson St. Paul's Churchyard (1798).

[34] P. Marcati, On the global stability of the logistic age dependent population growth. J. Math. Biol. 15 (1982) $215-226$.

[35] A.G. McKendrick, Applications of mathematics to medical problems. Proc. Edin. Math. Soc. 54 (1926) 98-130.

[36] S. Méléard and S. Roelly, Sur les convergences étroite ou vague de processus à valeurs mesures. C.R. Acad. Sci. Paris, Série I 317 (1993) 785-788.

[37] S. Méléard and V.C. Tran. Age-structured trait substitution sequence process and canonical equation. Submitted.

[38] S.P. Meyn and R.L. Tweedie, Stability of markovian processes iii: Foster-lyapunov criteria for continuous-time processes. Advances in Applied Probability 25 (1993) 518-548.

[39] K. Oelschläger, Limit theorem for age-structured populations. Ann. Probab. (1990).

[40] G. Da Prato and J. Zabczyk, Stochastic Equations in Infinite Dimensions. Cambridge Uiversity Press (1992).

[41] S.T. Rachev, Probability Metrics and the Stability of Stochastic Models, John Wiley \& Sons (1991).

[42] M.M. Rao and Z.D. Ren, Theory of Orlicz spaces. M. Dekker, New York (1991).

[43] S. Roelly, A criterion of convergence of measure-valued processes: Application to measure branching processes. Stochastics 17 (1986) 43-65.

[44] W. Rudin, Real and Complex Analysis. McGraw-Hill International Editions, third edition (1987).

[45] F.R. Sharpe and A.J. Lotka, A problem in age distribution. Philos. Mag. 21 (1911) 435-438.

[46] W. Solomon, Representation and approximation of large population age distributions using poisson random measures. Stochastic Process. Appl. 26 (1987) 237-255.

[47] V.C. Tran, Modèles particulaires stochastiques pour des problèmes d'évolution adaptative et pour l'approximation de solutions statistiques. Ph.D. thesis, Université Paris X - Nanterre. http://tel.archives-ouvertes.fr/tel-00125100.

[48] P.F. Verhulst, Notice sur la loi que la population suit dans son accroissement. Correspondance Mathématique et Physique 10 (1838) 113-121.

[49] C. Villani, Topics in Optimal Transportation. American Mathematical Society (2003).

[50] F.J.S. Wang, A central limit theorem for age- and density-dependent population processes. Stochastic Process. Appl. 5 (1977) 173-193.

[51] G.F. Webb, Theory of Nonlinear Age-Dependent Population Dynamics, Monographs and Textbooks in Pure and Applied mathematics 89, Marcel Dekker, inc., New York-Basel (1985).

[52] C. Zuily and H. Queffélec, Éléments d'analyse pour l'agrégation. Masson (1995). 